Digital version, 20th December 2010.

\title{
Optimization of Comb-Drive Actuators
}

Nanopositioners for Probe-based Data Storage and

Musical MEMS

\author{
JOHAN B. C. ENGELEN
}




\section{Graduation committee}

Prof. dr. ir. Ton J. Mouthaan

University of Twente (chairman and secretary)

Prof. dr. Miko C. Elwenspoek

Dr. ir. Leon Abelmann

University of Twente (promotor)

Prof. dr. Gary K. Fedder

Prof. dr. Pieter H. Hartel

University of Twente (assistant promotor)

Carnegie Mellon University, Pittsburgh, PA, USA

University of Twente

Prof. dr. ir. Fred van Keulen

Prof. dr. ir. Gijs J. M. Krijnen

Delft University of Technology, the Netherlands

Dr. Mark A. Lantz

University of Twente

Prof. dr. ir. Stefano Stramigioli

IBM Zürich Research Laboratory, Switzerland

University of Twente

\section{Paranymphs}

Wabe W. Koelmans

Léon A. Woldering

$$
\text { II MESA+ }
$$

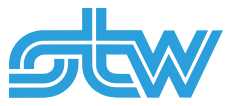

\section{UNIVERSITY OF TWENTE.}

The research presented in this dissertation was carried out at the Transducers Science \& Technology group at the $\mathrm{MESA}^{+}$Institute for Nanotechnology at the University of Twente, Enschede, the Netherlands. The project is supported by the Dutch Technology Foundation STW, which is the applied science division of NWO, and the Technology Programme of the Ministry of Economic Affairs ("A4 - Adaptive non-volatile storAge systems for Adaptive Applications", project number TES.06369)

E-mail: j.b.c.engelen@alumnus.utwente.nl

On the cover, a micronium wafer (front), where the instrument's resonators appear coloured because the incident light is diffracted by the perforation of the resonator masses; and a portrait of the author (back), seen through the lithography mask used to fabricate the micronium. Photos by Arvid Jense.

Printed by Gildeprint Drukkerijen, Enschede, the Netherlands Copyright (C) 2010 Johan B. C. Engelen, Enschede, the Netherlands

Some rights reserved. All or part of this book may be reproduced or transmitted in any form or by any means, electronic or mechanical, including photocopying, or by any information storage or retrieval system, without permission in writing from the author, only if proper reference to the original work is made.

ISBN 978-90-365-3120-7

DOI $10.3990 / 1.9789036531207$ 


\title{
Optimization of Comb-Drive Actuators Nanopositioners for Probe-based Data Storage AND Musical MEMS
}

\author{
DISSERTATION
}

to obtain

the degree of doctor at the University of Twente, on the authority of the rector magnificus, prof. dr. H. Brinksma, on account of the decision of the graduation committee, to be publicly defended on Friday, 14 January 2011 at 13:15

by

\section{Johannes Bernardus Charles Engelen}

born on 8 June 1983, in Nijmegen, the Netherlands 
This dissertation is approved by

Prof. dr. Miko C. Elwenspoek University of Twente (promotor)

Dr. ir. Leon Abelmann University of Twente (assistant promotor) 
THE ERA OF INFINITE STORAGE

Imagine carrying all the music ever recorded by the human race in your pocket. That will be possible by the end of this decade. If you want all the movies and TV programs, too, that will take only a few years more. Or imagine making an audio recording of your whole life, from beginning to end: that is affordable already. Video will be possible in a few years. Data storage devices such as hard drives and flash memory have gotten so dense and so cheap that for most purposes their storage capacity will soon be unlimited.

The era of infinite storage is about to begin.

Felten, 2010 



\section{Contents}

The era of infinite storage i

Contents

1 Introduction 1

1.1 Parallel probe-based data storage $\ldots \ldots \ldots 2$

2 Scanners for probe data storage 5

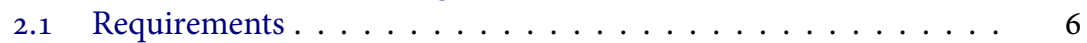

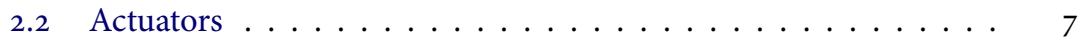

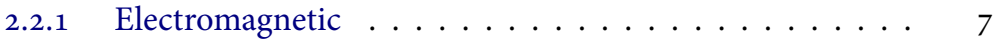

2.2.2 Electrostatic comb drives . . . . . . . . . . . . 10

2.2.3 Electrostatic stepper motors . . . . . . . . . . 14

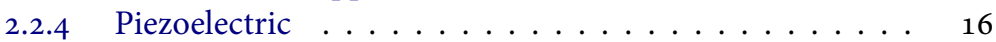

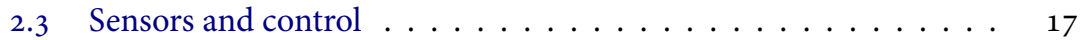

2.4 Conclusion . . . . . . . . . . . . . . . . . . . 19

3 A mass-balanced electrostatic $x / y$-scanner 21

3.1 Design . . . . . . . . . . . . . . . . 22

3.2 Fabrication ........................ 25

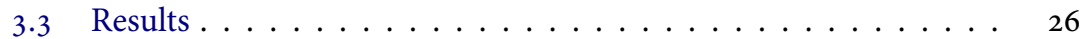

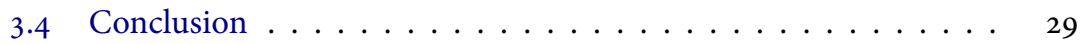

3.5 Acknowledgements .................. 30

4 Optimized comb-drive finger shape for shock-resistant actuation 31

4.1 Theory .......................... 34

4.1.1 Analytical model . . . . . . . . . . . . . . . 34

4.1.2 Graphical determination of equilibrium stability . . . . 36

4.1 .3 Design of optimal shape . . . . . . . . . . . 38

4.1 .4 Asymmetric shape . . . . . . . . . . . . . . 40

4.2 Finite-element analysis . . . . . . . . . . . . . . . . . . 41

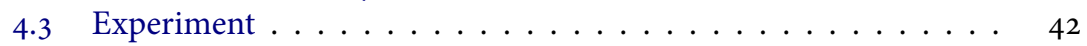

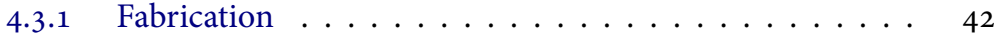

4.3 .2 Measurement results . . . . . . . . . . . 47 


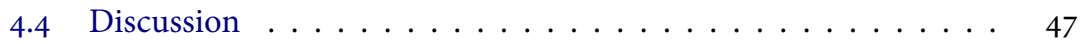

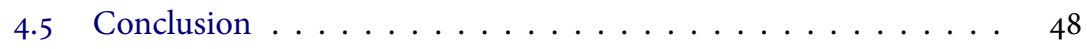

4.6 Acknowledgements .................... 49

5 Energy consumption comparison $\quad 51$

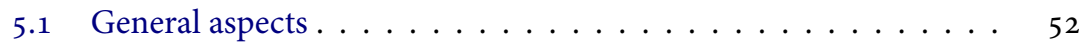

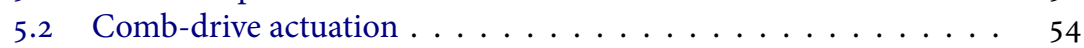

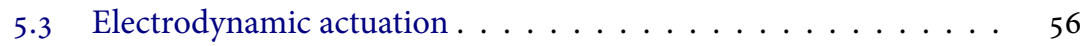

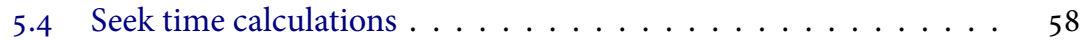

5.4 .1 Comb drives with 'optim' fingers . . . . . . . . . . . . . . 59

5.4 .2 Electrodynamic actuator . . . . . . . . . . . 59

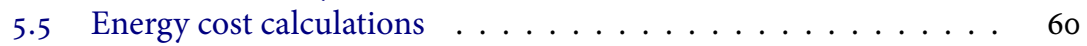

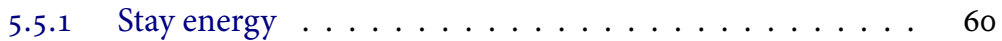

5.5 .2 Read/write energy ................ 61

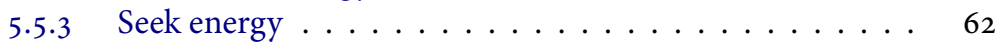

5.6 Results for hypothetical designs . . . . . . . . . . . . . . . 65

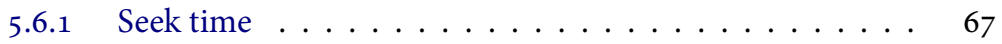

5.6 .2 Stay energy $\ldots \ldots \ldots \ldots \ldots \ldots \ldots \ldots$

5.6 .3 Read/write energy . . . . . . . . . . . . . . 68

5.6 .4 Seek energy . . . . . . . . . . . . . . . 69

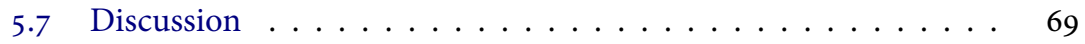

5.8 Conclusion ........................ 71

6 The Micronium - a musical MEMS instrument 73

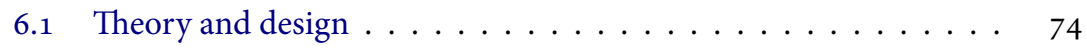

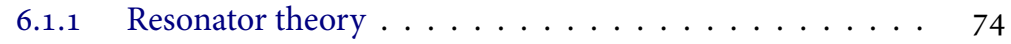

6.1 .2 Instrument design $\ldots \ldots \ldots \ldots \ldots \ldots$

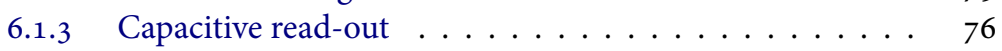

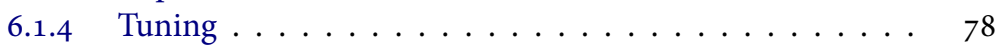

6.2 Experimental details and results . . . . . . . . . . . 79

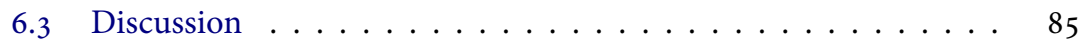

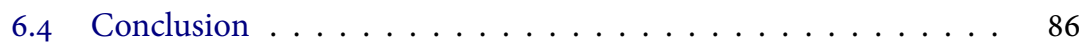

6.5 The Micronium project . . . . . . . . . . . . . . . . . 87

6.6 Acknowledgements .................. 88

$\begin{array}{llr}7 & \text { Conclusion and future outlook } & 89\end{array}$

$\begin{array}{ll}\text { Appendices } & 93\end{array}$

A Optimal charging of a capacitor 95

A.1 Without leakage resistor . . . . . . . . . . . . . . . . 95

A.2 With leakage resistor ................. 95

B Seek time for electrodynamic actuator $\quad 99$

$\begin{array}{lll}\text { C Process Flow } & 103\end{array}$ 
Bibliography 108

Summary

118

Samenvatting

119

Publications

120

Dankwoord

122

Biography / Biografie

124 



\section{Chapter 1}

\section{Introduction}

Far out in the uncharted backwaters of the unfashionable end of the western spiral arm of the Galaxy lies a small unregarded yellow sun. Orbiting this at a distance of roughly ninety-eight million miles is an utterly insignificant little blue-green planet whose ape-descended life forms are so amazingly primitive that they still think digital watches are a pretty neat idea (Adams, 1979).

This planet has a problem, which is this: data storage.

The importance of storing information is eminent; it defines a person's character, a people's culture, it defines humanity. Examples of human information storage are books, recipes, photographs, clay tablets, mathematics, audio cassettes, recollections, money, cairns *, maps, and paintings. Technological advances have made it possible to convert (an approximation of) the information in all these examples, except recollections, to digital information: a stream of 'ones' and 'zeros'. With the increasing possibilities of digitizing information, so does the demand for data storage capacity. A study by the International Data Corporation states that

"As forecast, the amount of information created, captured, or replicated exceeded available storage for the first time in 2007. Not all information created and transmitted gets stored, but by 2011, almost half of the digital universe will not have a permanent home." - Gantz et al., 2008, p.2

Hence the problem.

Since 2007, it is no longer possible to store all created information if we wanted to, and continuous data loss has become unavoidable. Not all created information is important enough for storage (how to decide?), but there is enough information that is deemed important for a continued demand for increased data storage capacity.

Over the past decades, data storage capacity has increased steadily, while the cost per bit has decreased. The three main rewritable data storage media are tape drives, hard disks, and flash memory. Hard disks and flash memory are already designed close to fundamental limits, and further scaling will be difficult and costly. Flash memory may soon be replaced by another solid-state memory technology,

\footnotetext{
*'steenmannetjes' in Dutch
} 
phase-change memory (PCRAM). A parallel probe-based data storage system is one of the promising candidates to continue the growth of data storage capacity.

Tape drives have the lowest cost per bit, because the tape is very thin and rolled up on a reel, resulting in a large volumetric capacity despite the relatively low data density compared to hard disks. Because of the relatively low data density, the data capacity can be increased without reaching fundamental physical limits in the near future. However, tape drives have slow access times due to their sequential storage. Their use is therefore limited to archival storage.

The hard disk drive roadmap largely depends on the superparamagnetic limit of its media: as bit volumes decrease, the energy stored in one bit becomes comparable to its thermal energy, and the magnetization state becomes unstable. Because the current data density is high, increasing the data density has become a large problem for the hard disk industry. Moreover, the decrease in access time has not kept pace with the increase in areal density. The access time is limited by the fact that a single head is addressing an increasing amount of data as the areal density (and therefore disk capacity) increases. Furthermore, the access time is limited by the disk rotational speed.

The density of solid state memory depends on the resolution of the electrical interconnections used to address the individual bit (or groups of bit levels, as in multi level recording) through a planar silicon manufacturing process. Solid state memorys evolutionary roadmap is therefore driven by the ability to decrease the minimum lithographic feature size, which in turn is fuelled by continuous investments in new costly semiconductor factories. At the time of publication a NAND flash manufacturing facility requires an investment of 3.4 billion dollars. Such an investment would generate less than $1 \%$ of the yearly shipped capacity of hard disk drives. Due to such huge capitalization it is unlikely that lithographically defined patterned media or solid-state disks will replace a substantial amount of hard disk drive production (Fontana and Hetzler, 2009).

Parallel probe-based data storage, or probe (data) storage, overcomes the limitations of magnetic hard disks and flash memory, because the ultimate bit size is neither lithographically defined nor bound by the superparamagnetic limit, but essentially limited by a size of a few atoms. Although data is mechanically addressed, the access time in probe storage benefits from the smaller required displacements of the read/write heads relative to the media, compared with hard disks. Parallel probe operation allows data rates to be competitive with hard disks and flash memory. The economics and performance of probe storage lie between the hard disk (relatively cheap but slow) and flash memory (relatively expensive but fast).

\subsection{Parallel probe-based data storage}

Parallel probe-based data storage combines two important developments of the last decades; (1) the invention of the Scanning Tunneling Microscope by Binnig and Rohrer (Binning et al., 1982, note the spelling error!), which triggered the very fruitful field of scanning probe microscopy (SPM); and (2) the industrial maturity 


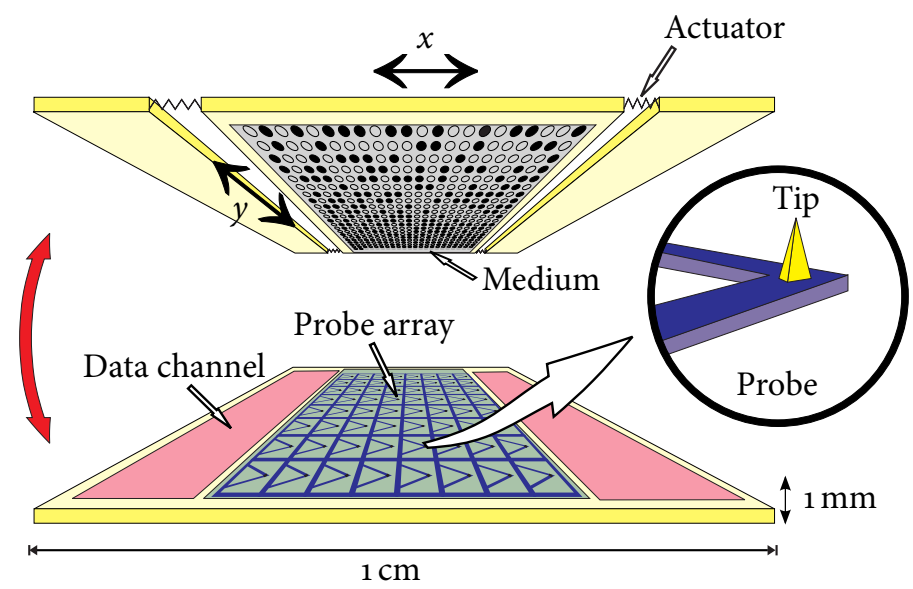

FIGURE 1.1 - Overview of a parallel probe-based data storage system. AFM-like probes are used to read and write data. The medium on the platform, that can move in $x$ and $y$ directions, stores data.

of silicon-based microelectromechanical systems (MEMS). It is now practicable, for example, to use SPM technology to modify the surfaces of materials on the nanoscale, rather than just for microscopic imaging. Such surface modification might comprise the writing and reading of data, providing a storage system with, ultimately, atomic resolution. Indeed, such atomic resolution was demonstrated in 1990 by Eigler and Schweizer (1990), who placed individual Xe atoms on a single-crystal nickel substrate to spell out the IBM logo. However this approach was exceedingly slow and, from a system perspective, offered an impracticably low data rate. A much higher data rate was achieved at IBM Almaden by using a heated atomic force microscope (AFM) probe mounted over a rotating disk with a polymer film. Data was written by creating small indents, at impressive data rates over $10 \mathrm{Mbit} \mathrm{s}^{-1}$ (Mamin et al., 1995, 1999), but still too slow for commercial applications. Since the active part of AFM probes have dimensions in the order of $100 \mu \mathrm{m}$, the logical step forward is to use arrays of probes to multiply the data rate. Dense stacking of probes was made possible by micromachining technology (Bustillo et al., 1998), which developed from integrated circuit technology following Feynman's famous foreseeing lecture "There's plenty of room at the bottom" (Feynman, 1959). The manufacturing technologies had matured by the end of the last century, entering the 'VLSI age of MEMS' (as Binnig calls it) with the development of arrays of AFM probes (Minne et al., 1998), and integrated nano-positioning systems (Cheung et al., 1996).

A schematic overview of a probe data storage system is shown in Figure 1.1. A moving platform carrying the storage medium is suspended above an array of probes. This platform is called the medium sled and is actuated in the lateral $x$ and $y$ directions to position the storage medium relative to the array of probes. 
The probes in the array 'feel' the storage medium, and are operated individually in parallel, reading and writing data. The storage medium can be a polymer, storing data topographically (Pantazi et al., 2008), or a phase change medium, storing data in the conductivity modified by local phase changes of the medium (Gidon et al., 2004), or magnetic, storing data in the magnetization (Ohkubo et al., 1993), or ferroelectric, storing data in the polarization (Hidaka et al., 1996). Atomic-scale storage without continuous thin film has been shown by Bennewitz et al. (2002), storing data in $\mathrm{Si}$ atoms on top of a reconstructed silicon surface. The probes are specifically designed for the type of storage medium. The positioning subsystem of actuators, position sensors, and control circuitry, is referred to as the nanopositioner or scanner. The medium and probes mainly determine the data density, and so the capacity. The positioning system determines the access and seek time, and therefore the performance of the system.

Several different actuator types have been proposed for the scanner, as is discussed in Chapter 2. Currently, the only published probe data storage prototype uses an electrodynamic actuator. We believe that electrostatic comb-drive actuation is better suited for a probe data storage device, offering less energy consumption and easier fabrication. This thesis investigates the application of electrostatic combdrives in scanners for probe data storage in comparison with other actuation types. Chapter 3 discusses the replacement of the electrodynamic actuators in IBM's nanopositioner by comb drives. The shape of a comb drive can be tailored to obtain a specific force characteristic; the optimal comb-drive shape for use in a probe storage scanner is presented in Chapter 4. The energy consumption of comb drive actuation is compared with electromagnetic actuators in Chapter 5. Finally, Chapter 6 shows how comb drives can be used to create a musical instrument in MEMS, to promote public understanding of MEMS. 


\section{Chapter 2}

\section{Scanners for probe data storage}

A nanopositioner controls the position of an object with an accuracy on the order of nanometres. A probe data storage system requires a 2D nanopositioner, referred to as 'the scanner', to move the storage medium relative to the probe array. Hubbard et al. (2006) have written a summary of actuators for nanopositioners published in the literature, comparing piezoelectric, magnetic, electrostatic, thermal, electrochemical and 'inchworm* actuators. Although an actuator for a nanopositioner need not be small, most of the discussed actuators are, and are fabricated using microelectromechanical systems (MEMS) technology. A comparison of specifically MEMS actuators was written by Bell et al. (2005), with an emphasis on selecting a certain actuator type for a specific purpose. Bell et al. (2005) show that there are several MEMS actuator types suitable for probe data storage, where the required range is on the order of $100 \mu \mathrm{m}$ with nanometre resolution, and a maximum force on the order of millinewtons. Electromagnetic, electrostatic (comb drive, dipole surface drive, inchworm), thermal, and piezoelectric actuators all seem promising candidates for use in a probe data storage system, and scanner designs have been published for all these actuator types except the thermal type. Perhaps, the reason for the absence of thermally actuated scanners for probe data storage is the large required power for fast thermal actuators.

Below, first the requirements for the scanner in a probe data storage system are discussed, followed by a discussion of published actuators and scanners specifically designed for probe data storage. Finally, a brief overview of sensors and control for probe data storage will be given.

This chapter is based on the book chapter: M. Gemelli, L. Abelmann, J. B. C. Engelen, M. G. Khatib, W. W. Koelmans, and O. Zaboronski, "Mass Storage using Milliped Technology", Memory Mass Storage, ISBN 978-3-642-14751-7, Springer Verlag, 2011.

* "An inchworm moves by gripping a surface with its hind legs while retracting its body," Hubbard et al. (2006) 


\subsection{Requirements}

In order to access data at different positions, a positioning system is required. The positioning system should be able to move in two perpendicular directions ( $x$ and $y$ ) with nanometre precision. Actuation in the third $z$-direction is usually not present in the positioning system, as it is generally part of the probe (array) design. Although arguments can be mounted in favour of having a separate positioning system for each probe, all systems are designed such that there is a single moving platform for the complete device. Most designs ${ }^{\dagger}$ feature a stationary probe array and a moving medium sled, or 'scan table', to prevent exciting unwanted vibrations in the probes during servoing and (perhaps more important) to circumvent the problem of how to route many wires to a moving suspended array of many probes. The separation of probe array and storage medium with positioning system greatly simplifies the design; the wafers with the probe array and positioning system can be fabricated separately and bonded together afterwards.

There should be no restriction in the movement trajectory. Most often, a raster pattern is employed for reading and writing, scanning back and forth in the horizontal direction, while making steps in the vertical direction. But at all times, it should be possible to quickly move, or 'seek', to an arbitrary new location to start reading or writing. Moreover, instead of using a raster pattern, an outward spiral trajectory similar to a Compact Disc (CD) may be used (Mahmood and Moheimani, 2009).

To fully utilize the storage medium, the displacement range should be at least the size of the distance between probes in the array: currently on the order of $100 \mu \mathrm{m}$ (Despont et al., 2004), although this may decrease in the future. If overlapping probe fields are desired, the displacement range should be larger. The required positioning accuracy is on the nanometre scale, depending on the bit size $(<25 \mathrm{~nm}$ at a density of 1 Tbit in $^{-2}$ ) and the allowable bit-error-rate (Pozidis et al., 2004; Sebastian et al., 2007). For example, Pozidis et al. (2004) from IBM showed that at $641 \mathrm{Gbit} \mathrm{in}^{-2}$, a $2 \mathrm{~nm}$ displacement from the track centreline results in a 5 times larger raw bit error rate. For the $64 \times 64$ probe array described by Despont et al. (2004), a medium sled size of at least $6.4 \mathrm{~mm} \times 6.4 \mathrm{~mm}$ is required. The areal efficiency is defined as the ratio between medium sled size and total device size.

The performance in terms of access speed is determined by the actuator's maximum acceleration, sensor bandwidth and control circuit. The maximum acceleration should be large, not only for faster seek access, but also so that it is possible to quickly reverse motion at the end of each scan line if a raster pattern is used. Because a higher read/write speed will lead to a longer turn-around time, there is an optimal read/write speed and a corresponding optimal data rate (Carley et al., 2001). A higher acceleration means a higher continuous data rate can be reached, either by increasing the movement speed or by decreasing the turn-around time. Increasing the acceleration capability by reducing the medium sled mass must be done with care, so that the sled is still mechanically rigid enough for reliable probe reading

\footnotetext{
${ }^{\dagger}$ A notable exception is the design described by Yang et al. (2007) from the Data Storage Institute in Singapore, where the probe array is moved by a $1 \mathrm{D}$ linear motor.
} 
and writing. Increasing the maximum acceleration by increasing the maximum force is limited to what the power budget allows and what is physically possible without damaging the device (e.g. breakdown voltage or electromigration limit to the current). An access time on the order of $1 \mathrm{~ms}$ is required to compete with Flash cards or solid-sate disks ( $\sim 0.2 \mathrm{~ms})$ and hard disk drives $(\sim 4 \mathrm{~ms})$.

To perform seek operations or compensate for residual shock forces, the actuation force at any given position must exceed the suspension spring restoring force. The available force, equal to the actuator force minus the suspension spring force, if a function of position, and is larger at small displacements than at large displacements for most actuators. The minimum value of the available force is an important measure of how shock-resistant the design is, and of how fast the actuator can accelerate. Especially for mobile applications, shock-resistance is important (Lantz et al., 2007), requiring a large enough minimum value of the available force over the operation range.

The scanner should be able to scan back and forth many times without breaking down. For example, at $1 \mathrm{Tbit}^{-2}$, one full track of $100 \mu \mathrm{m}$ equals 3937 bits and there are 3937 tracks (assuming that the distance between bits is equal to the distance between tracks for ease of calculation). It will take 1969 cycles (back and forth) just to read all data once. The required number of cycles during the total life time of the scanner will exceed $10^{8}$ cycles.

Finally, there may be a certain power limit for the complete positioning system. The actuator should be energy efficient, especially for mobile applications. Although certain types of actuators require very little power for themselves (and seem attractive), their driving or control circuitry may require substantial power, leading to a high overall energy consumption. For a fair comparison of required power, one should view the positioning system as a whole.

\subsection{Actuators}

The reviews by Bell et al. (2005) and Hubbard et al. (2006) show that several physical actuation principles may be used in a probe-storage device. Different types of scanners, that have been designed specifically for probe storage, are found in the literature. It is yet unclear which actuation type is best suited for probe storage. Research focuses on MEMS actuators because they have a large ratio of in-plane motion range to device volume compared to macro actuators. Agarwal et al. (2006) show a comparison in terms of 'specific work' (force times travel range divided by footprint); different types of MEMS actuators all score about $10 \mu \mathrm{N} \mathrm{mm}^{-1}$, an order of magnitude lower than conventional 'milli-actuators'. It shows the challenging problem of creating MEMS actuators with large force, large displacement and small footprint.

\subsubsection{Electromagnetic}

Electromagnetic scanners use a coil to generate a magnetic field that leads to a force. All electromagnetic actuators designed for probe storage reported in the literature 
use a permanent magnet and a coil. It is possible to make an electromagnetic combdrive actuator without permanent magnet (Schonhardt et al., 2008), which will be discussed in Chapter 5, but it has not been used in a scanner design yet. To distinguish electromagnetic actuators with a permanent magnet from scanners without, actuators with permanent magnet are referred to as electrodynamic actuators.

An advantage of electrodynamic scanners is the relatively straightforward linear actuator design (linear displacement $v$ s. current curve), simplifying controller design. Another advantage for mobile probe storage is that an electrodynamic scanner, because it is current driven, can operate at the generally low available voltage of about $3.6 \mathrm{~V}$. A disadvantage is that permanent magnets are needed. Assembling an electrodynamic scanner will therefore be more complicated than assembling, for instance, an electrostatic comb-drive scanner. It also means that it is difficult to make the scanner very thin. The energy consumption of electromagnetic scanners in general is relatively large because of the relatively large required currents compared with the series resistance of the coils.

In 2000, Rothuizen et al. from IBM reported their proof-of-concept electrodynamic scanner for probe data storage (see Figure 2.1(a)): a five degree of freedom $x / y / z$-scanner, including tilt about the $x$ and $y$ axes, fabricated from silicon and electroplated copper springs and coils. The scanner contains a $2 \mathrm{~cm} \times 2 \mathrm{~cm} \mathrm{mo-}$ ving platform, held within a $3 \mathrm{~cm} \times 3 \mathrm{~cm}$ outer frame. The displacement range is $\pm 100 \mu \mathrm{m}$, however the required power of about $200 \mathrm{~mW}$ is very high. An improved design was reported two years later (Rothuizen et al., 2002, see Figure 2.1(b)), fabricated from a $200 \mu \mathrm{m}$ thick SU-8 layer. The design uses a similar configuration for coil and magnet. It improves on power dissipation ( $3 \mathrm{~mW}$ at $100 \mu \mathrm{m}$ displacement), on fabrication cost, and on compactness by placing the spring system below the moving platform. Two years later, a radical change in design was reported (Lantz et al., 2007; Pantazi et al., 2004, see Figure 2.1(c)). The new design is fabricated from a $400 \mu \mathrm{m}$ thick silicon wafer by deep reactive-ion etching through the full thickness of the wafer, the design being an extrusion of a two-dimensional layout. It features a mass-balancing concept to render the system stiff for external shocks while being compliant for actuation, such that the power dissipation is low. The actuator and scan table masses are linked via a rotation point, enforcing their movement in mutually opposite directions: when the actuator moves up, the table moves down and visa versa. External shocks exert inertial forces on the actuator and scan table mass, but, because the directions of the inertial forces are equal, they cancel each other through the rotation point (the scan table and actuator cannot move in the same direction at the same time). Because the springs are $400 \mu \mathrm{m}$ high (wafer thickness), the stiffness in the $z$-direction is large for passive shock rejection. Coils and magnets are glued manually onto the device. The actuator generates a force of $62 \mu \mathrm{N} \mathrm{mA}^{-1}$. Its power usage at $50 \mu \mathrm{m}$ displacement is $60 \mathrm{~mW}$ (80 $\mathrm{mA}$ current); this has been improved to about $7 \mathrm{~mA}$ and $2 \mathrm{~mW}$ power ${ }^{\ddagger}$. The medium sled is $6.8 \mathrm{~mm} \times 6.8 \mathrm{~mm}$, while the complete device is $16 \mathrm{~mm} \times 16 \mathrm{~mm}$; the areal efficiency is about $25 \%$ and has decreased dramatically in comparison to

\footnotetext{
${ }^{\ddagger}$ private communication with Mark A. Lantz, IBM Zürich
} 


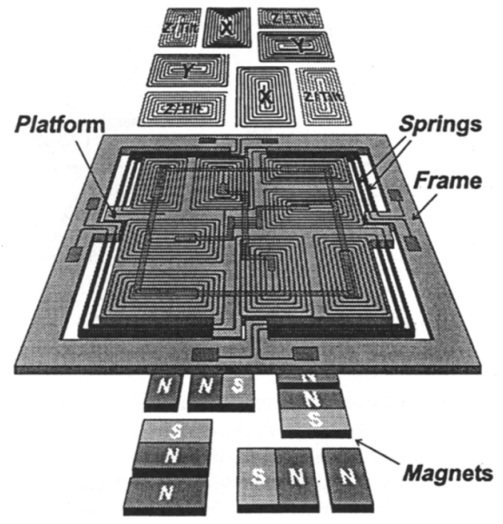

(a) Scan table is $2 \mathrm{~cm} \times 2 \mathrm{~cm}$ large and has 5 DOF (Rothuizen et al., 200o).

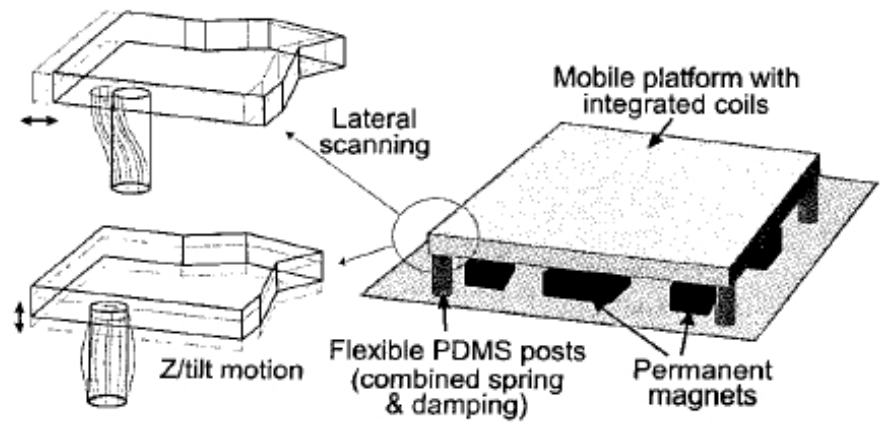

(b) Fabricated from $S U-8,1.5 \mathrm{~cm} \times 1.5 \mathrm{~cm}$ scan table (Rothuizen et al., 2002).

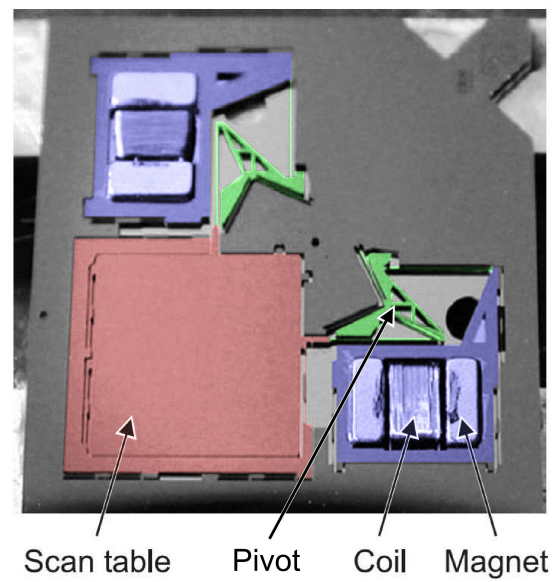

(c) Mass-balanced and $x / y$ only, $6.8 \mathrm{~mm} \times 6.8 \mathrm{~mm}$ scan table (Pantazi et al., 2004).

FIGURE 2.1 - The evolution of IBM's electrodynamic scanner. 


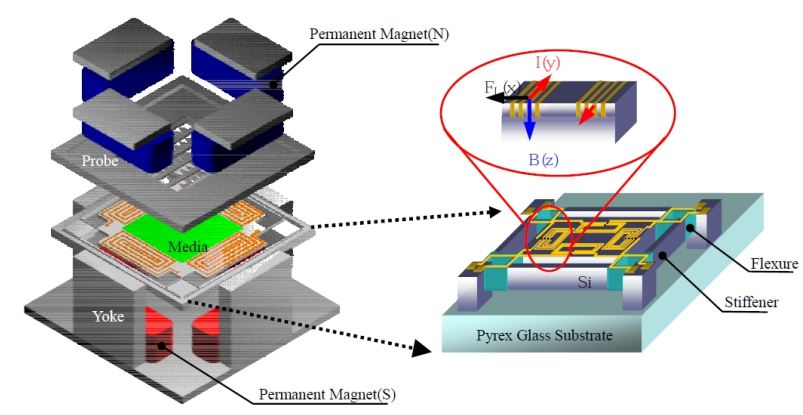

FIGURE 2.2 - The electrodynamic scanner by Samsung (Choi et al., 2001a). The size of the total device and scan table are $13 \mathrm{~mm} \times 13 \mathrm{~mm}$ and $5 \mathrm{~mm} \times 5 \mathrm{~mm}$, respectively.

the earlier designs. The in-plane resonance frequencies lie around $150 \mathrm{~Hz}$; the first out-of-plane resonance frequency lies an order of magnitude higher.

Another electrodynamic scanner was reported in 2001 by Choi et al. $(2001 a, b)$ from Samsung, see Figure 2.2. The scanner is fabricated from silicon; the coils are made by filling high aspect-ratio silicon trenches. The medium sled size equals $5 \mathrm{~mm} \times 5 \mathrm{~mm}$. The displacement of $13 \mu \mathrm{m}$ at $80 \mathrm{~mA}$ is smaller than the scanner by IBM, however this was measured without top magnets and yokes planned in the design to increase the magnetic field and force. The measured in-plane resonances are $325 \mathrm{~Hz}$ (translational) and $610 \mathrm{~Hz}$ (rotational).

A plastic electrodynamic scanner is described by Huang et al. (2010a) from Seagate, however not much design and fabrication details are available. The scanner has three degrees of freedom: $x / y$ translation and rotation about the $z$ axis. The $\pm 150 \mu \mathrm{m}$ displacement range is large, but the resonance frequency is only $70 \mathrm{~Hz}$.

\subsubsection{Electrostatic comb drives}

Electrostatic comb drives (Tang et al., 1989) are microelectromechanical actuators that are often used in MEMS technology, because their design and fabrication are relatively straightforward, and because the electrostatic force becomes relatively large at small dimensions. A comb drive consists of two interdigitated structures, shaped like combs, that attract each other when they are charged oppositely by applying an external voltage. The generated force, however, is small and many fingers are needed to generate sufficient force, reducing the areal efficiency. Other electrostatic actuators like the dipole surface drive and shuffle drive (see \$2.2.3), can generate more force but are more complicated to manufacture and to control. The generated force is proportional to and in the direction of increasing capacitance; the combs attract each other because that increases the overlap between the comb fingers which increases the capacitance (see (3.1) in the next chapter). Note that the capacitance change is large when the gap between fingers decreases. Therefore 
usually, springs restrict motion in the transversal direction to prevent this 'side snapin' An electrostatic comb drive is a non-linear device as the force is proportional to the applied voltage squared. Because of this quadratic dependence, the polarity of the applied voltage is unimportant and the force is always attractive. In order to move a stage in both positive and negative direction, two or more electrostatic actuators are required $\$$. Because the spring suspension force depends linearly on the position, the displacement also depends quadratically on the applied voltage. This relation can be linearised by driving the comb drives in differential mode, reducing the force of one comb drive by at the same time applying a voltage on the comb drive pulling in the opposite direction (Grade et al., 2004).

The maximum force of an electrostatic actuator depends on its breakdown voltage (depending on the minimum gap size) and the maximum available voltage. The breakdown voltage of comb drives is above $300 \mathrm{~V}$ for common micrometresized gaps (Chen et al., 2006), and is usually higher than the available voltage. To increase the maximum voltage, DC/DC step-up conversion is used. Examples of DC/DC converters specifically designed for MEMS applications are a high voltage CMOS design by Saheb et al. (2007) that converts $3 \mathrm{~V}$ up to $380 \mathrm{~V}$ using an external coil, and a standard CMOS design by Hong and El-Gamal (2003) that is capable of converting $1.2 \mathrm{~V}$ to $14.8 \mathrm{~V}$ without external components.

Compared to electromagnetic actuators, the advantage of comb drive actuators is their ease of fabrication. Moreover, the energy consumption of comb drives is lower, however, the energy consumption of the driving (DC/DC conversion) circuitry must be taken into account for a fair comparison with other scanners. Although a comb drive is a non-linear actuator, driving one is much simpler than driving electrostatic stepper motors. Disadvantages include the generally high required voltage and the low areal efficiency due to the large area needed for comb fingers to generate sufficient force. The force output is highly dependent on the minimum gap size that can be fabricated; reduced gap sizes due to improved fabrication methods will result in stronger comb drive actuators. In practice, the maximum force and voltage are limited by side pull-in because of insufficient spring suspension stiffness.

Already in 1992, a nanopositioner with integrated tip was published by Yao et al. (1992). It featured electrostatic parallel-plate (gap-closing) actuators, moving the probe instead of the 'medium'. However, its displacement range of $200 \mathrm{~nm}$ at $55 \mathrm{~V}$ is very limited. Another early design by Cheung et al. (1996) from the University of California at Berkeley includes position sensing and control, obtaining a displacement of $8 \mu \mathrm{m}$ at just $3 \mathrm{~V}$. However, the actuator is too thin to carry a load.

In 2000, Carley et al. (2000) from Carnegie Mellon University described a comb drive actuated scanner for probe storage, which was originally designed for a vibratory-rate gyroscope (Carley et al., 2001). The scanner reaches $50 \mu \mathrm{m}$ displacement at $120 \mathrm{~V}$; it contains 800 fingers in total with $500 \mu \mathrm{m}$ height and $16 \mu \mathrm{m}$ gap. Alfaro

\footnotetext{
${ }^{\S}$ A single dipole surface drive or shuffle drive can move in both positive and negative direction. In the sense meant here, they should be thought of as compound actuators consisting of 3 or more small electrostatic gap-closing actuators.
} 


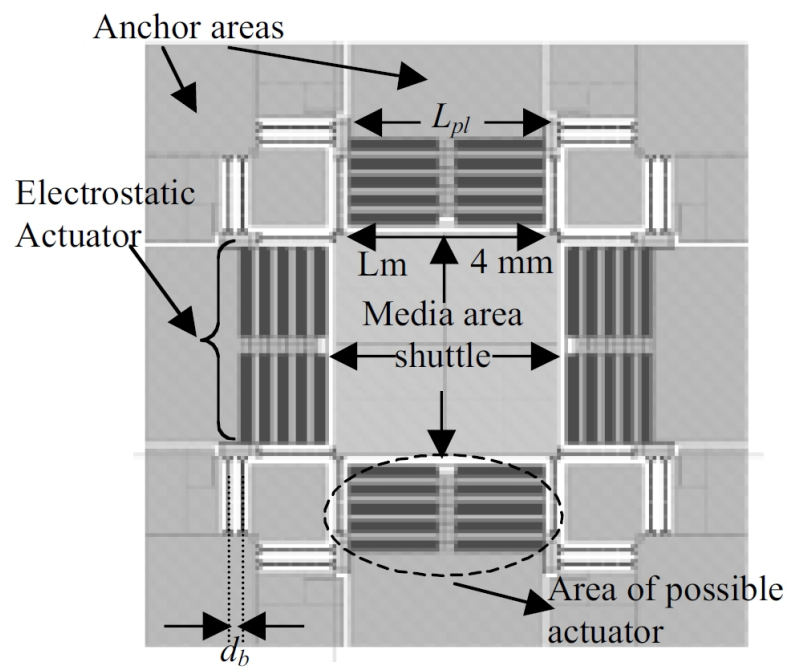

Figure 2.3 - The topology used by Alfaro and Fedder from Carnegie Mellon to find a scanner design with optimal footprint for a $\pm 50 \mu \mathrm{m}$ stroke (Alfaro and Fedder, 2002).

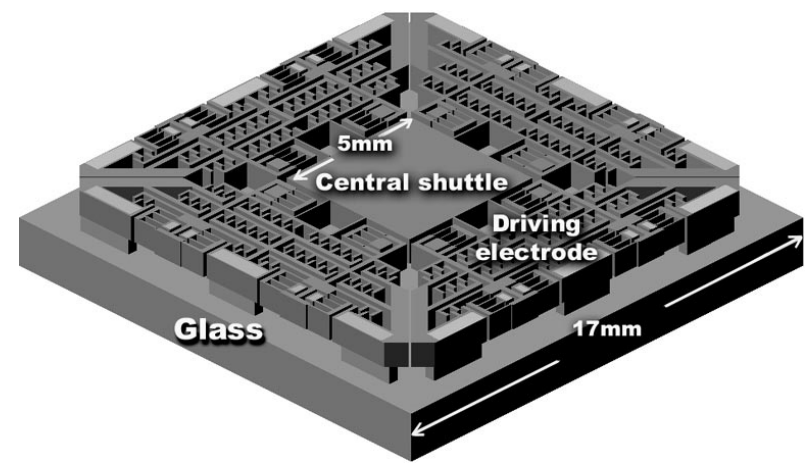

FIGURE 2.4 - Simplified layout of the micro XY-stage by Kim et al. (2003). The design features many fingers, generating a large force but reducing the areal efficiency to $11 \%$.

and Fedder (2002) from Carnegie Mellon University further improved this design using parametric optimization to optimize the footprint for $\pm 50 \mu \mathrm{m}$ stroke keeping the topology fixed (see Figure 2.3). Although Figure 2.3 is not drawn to scale, it is indicative of the generally low areal efficiency of comb drive scanners.

The comb-drive scanner reported by Kim et al. (2003) uses $275523 \mu \mathrm{m}$-thick fingers with $48 \mu \mathrm{m}$ height and $3 \mu \mathrm{m}$ gap (see Figure 2.4). These dimensions are common for comb drive designs, where the maximum aspect-ratio (height to gap ratio) 

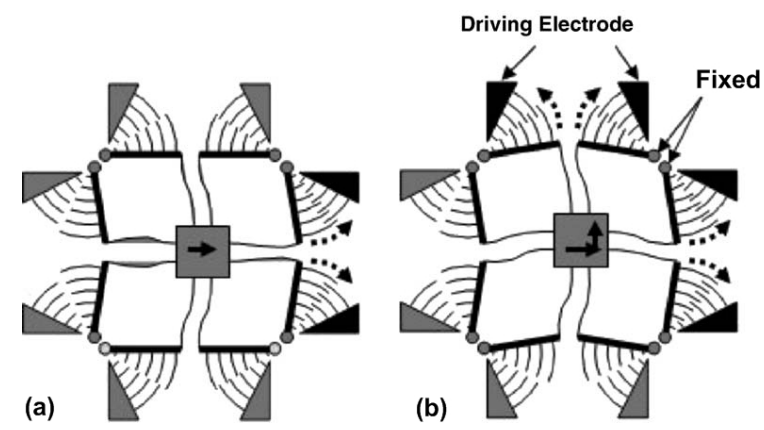

FIGURE 2.5 - Simplified model of the actuation principle used in the design by Kwon et al. (2006), featuring curved comb drives; (a) movement to the right by actuating the rotational comb drives on the right; (b) diagonal movement by actuating the comb drives on the right and top.

is limited to $\sim 20: 1$ by the deep reactive-ion etching fabrication process (Jansen et al., 2009). The scan table is $5 \mathrm{~mm} \times 5 \mathrm{~mm}$ large and $18 \mu \mathrm{m}$ static displacement is reached at $13.5 \mathrm{~V}$. Because of the large number of fingers, the force is large and the required voltage is low, however, it also leads to a low areal efficiency of approximately $11 \%$.

Kwon et al. (2006) published an $x / y$-scanner for optical applications, but the design could also be applied to probe storage. The design uses ' $L$ '-shaped suspension springs and rotational comb-drives (see Figure 2.5). Its displacement range is $55 \mu \mathrm{m}$ at $40 \mathrm{~V}$, however it is unclear whether the suspension is stiff enough for use in probe storage.

The somewhat unconventional positioning system design by Yang et al. (2007) from the Data Storage Institute in Singapore features an electrostatic comb-drive $x / y$-scanner (Lu et al., 2005) for precise positioning and a 1D miniature electromagnetic linear motor for course positioning of a 1D probe array (see Figure 2.6). By using an extra course positioner, a small number of probes in a 1D array can be used instead of a 2D probe array. The comb drive scanner features a scan table of $5 \mathrm{~mm} \times 5 \mathrm{~mm}$; the areal efficiency is $25 \%$. Simulations indicate a static displacement of $20 \mu \mathrm{m}$ at $55 \mathrm{~V}$, but no measurement results are reported.

All the comb drive scanners mentioned above directly link the actuators to the scan table. This means that in-plane shocks have to be actively compensated for by the actuators. Especially at large displacements, when the available force is low, this leads to a low shock rejection capability. The electrostatic scanner designs by Sasaki et al. (2008) use mass balancing for internal shock force rejection. Inertia forces due to fast acceleration in the $y$-direction may influence the comb finger gap in the $x$-direction, which may lead to instability in the comb drives for the $x$-direction. To cancel these inertia forces in the $y$-direction, the scan table is split into two plates of equal mass that are actuated in mutually opposite directions. External inertial forces are not cancelled as opposite movement is not mechanically enforced. The best of the three investigated designs reached static displacements of $110 \mu \mathrm{m}$ at $70 \mathrm{~V}$ 


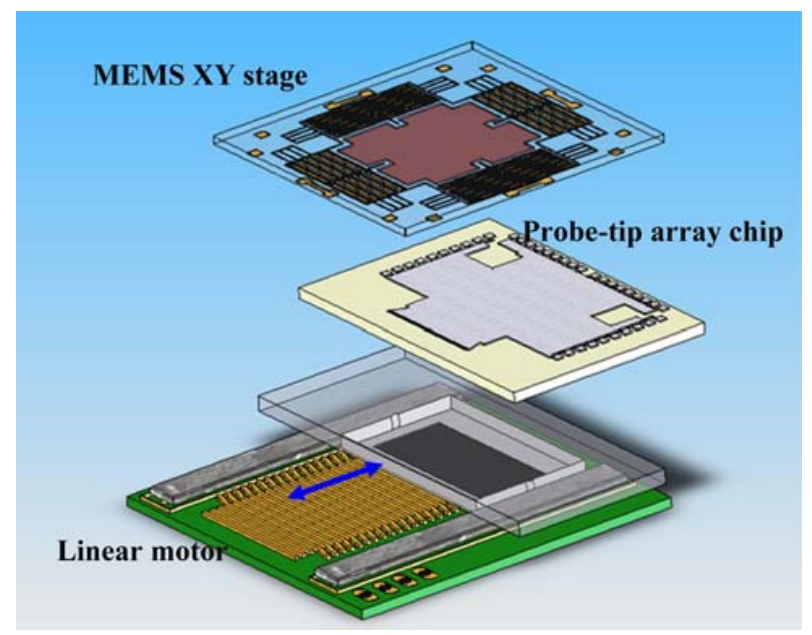

Figure 2.6 - A schematic of the design by Yang et al. (2007) from the Data Storage Institute in Singapore, featuring a comb-drive $x / y$-stage that carries the storage medium, and beneath it a miniature linear motor with a $1 \mathrm{D}$ probe array. The $M E M S$ stage's size is $1 \mathrm{~cm} \times 1 \mathrm{~cm}$.

and $90 \mu \mathrm{m}$ at $125 \mathrm{~V}$ for the $x$ and $y$ directions, respectively.

\subsubsection{Electrostatic stepper motors}

Figure 2.7 shows the electrostatic dipole surface drive by Agilent (Agarwal et al., 2006; Hoen et al., 2003). The translator features a periodic pattern of electrodes and is suspended above the stator, which also features a periodic pattern of electrodes but with a different pitch. Oppositely charged translator and stator electrodes will try to align with each other, increasing the capacitance between them, and creating a translational force. Sequentially applying a voltage on $V_{1}, V_{2}, \ldots, V_{7}$, in Figure 2.7 (b) results in a stepping motion of the translator to the left. The step size of $400 \mathrm{~nm}$ is determined by the difference in electrode pitch between stator and translator. Smaller displacements are made by adjusting the voltage on one of the electrodes. In the design reported by Agarwal et al. (2006), the gap between the stator and translator electrodes is $2.4 \mu \mathrm{m}$. A high out-of-plane to in-plane stiffness ratio is required, because the available force is limited by the vertical snap-in voltage ( $48 \mathrm{~V}$ in this case). A displacement of $17 \mu \mathrm{m}$ was reached with $30 \mathrm{~V}$ bias; larger displacements up to $70 \mu \mathrm{m}$ were reached, however in that case significant out-ofplane motion was observed. The out-of-plane force can be cancelled by another dipole surface drive 'upside-down' on top of the translator.

The dipole surface drive can potentially provide a large force at low voltages, because the capacitance (and thus force) between translator and stator electrodes can be made large due to the small gap size that is not limited by lithography or 


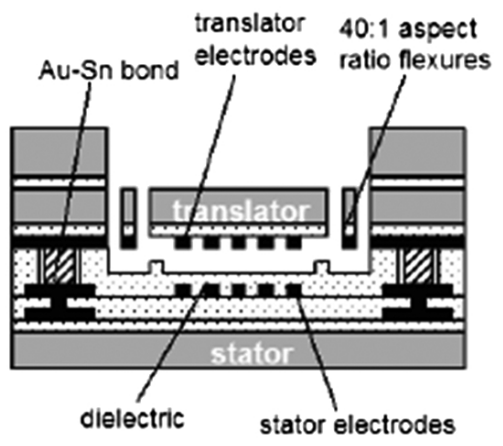

(a) Schematic cross-section of the dipole surface drive.

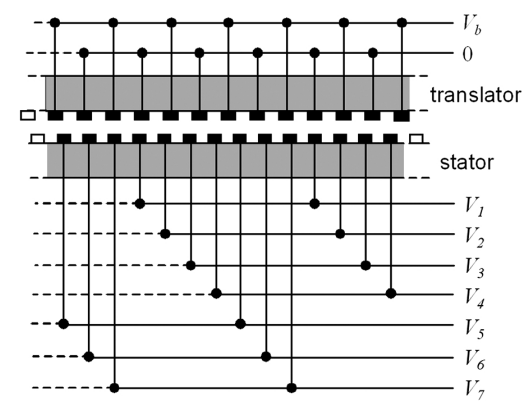

(b) Wiring diagram of the translator and stator electrodes.

Figure 2.7 - The dipole surface drive actuator by Agilent (Agarwal et al., 2006). The translator and stator electrodes have a different pitch, and by sequentially applying a voltage on $V_{1}, V_{2}, \ldots, V_{7}$, small steps to the left are made by the translator.

deep reactive-ion etching. Because the actuator is placed under the scan table, the areal efficiency is greatly increased over e.g. comb-drive designs. However, the fabrication process and drive circuitry are more complex than for electromagnetic actuators or electrostatic comb drives. To make the design more shock-resistant, perhaps a 2D mass-balancing scheme could be used similar to IBM's electrodynamic scanner (Lantz et al., 2007) without decreasing the areal efficiency.

Another electrostatic stepper motor is the 'inchworm' shuffle drive by Tas et al. (1998) from the University of Twente. The shuffle drive consists of two clamp 'feet' connected by a thin plate. The feet and plate can be pulled towards the bulk individually. Figure 2.8 shows how the motor makes a step. The actuator's output force and step size is determined by how much plate deformation is obtained when applying a voltage on the plate. The step size is on the order of tens of nanometres, but is highly dependent on the displacement due to the spring suspension's restoring force ( $\mathrm{Pa}$ trascu et al., 2007). The generated force is large, because the capacitance change of the parallel-plate gap-closing actuator is relatively large, and because the plate acts as a mechanical lever. Sarajlic et al. $(2005 b)$ describe a $200 \mu \mathrm{m} \times 1500 \mu \mathrm{m}$ shuffle motor with a $70 \mu \mathrm{m}$ displacement range and an output force of $0.45 \mathrm{mN}$ at driving voltages of $65 \mathrm{~V}$ and $150 \mathrm{~V}$ for the plate and clamps, respectively. A $482 \mu \mathrm{m} \times 482 \mu \mathrm{m}$ 2D shuffle motor reached displacements of $60 \mu \mathrm{m}$ (corresponding to $0.64 \mathrm{mN}$ force) at driving voltages of $45 \mathrm{~V}$ and $36 \mathrm{~V}$ for the plate and clamps, respectively, only being limited by the design layout (Sarajlic et al., 2005a). Unfortunately, stiction and friction of the feet are a large problem for reliable operation (Patrascu, 2006). Moreover, a complex control loop is required (Patrascu et al., 2007). Interestingly, because the device is electrostatically clamped to the base plate, it is inherently shock-resistant. But when the shock force plus the spring force exceeds the stiction force, there is 


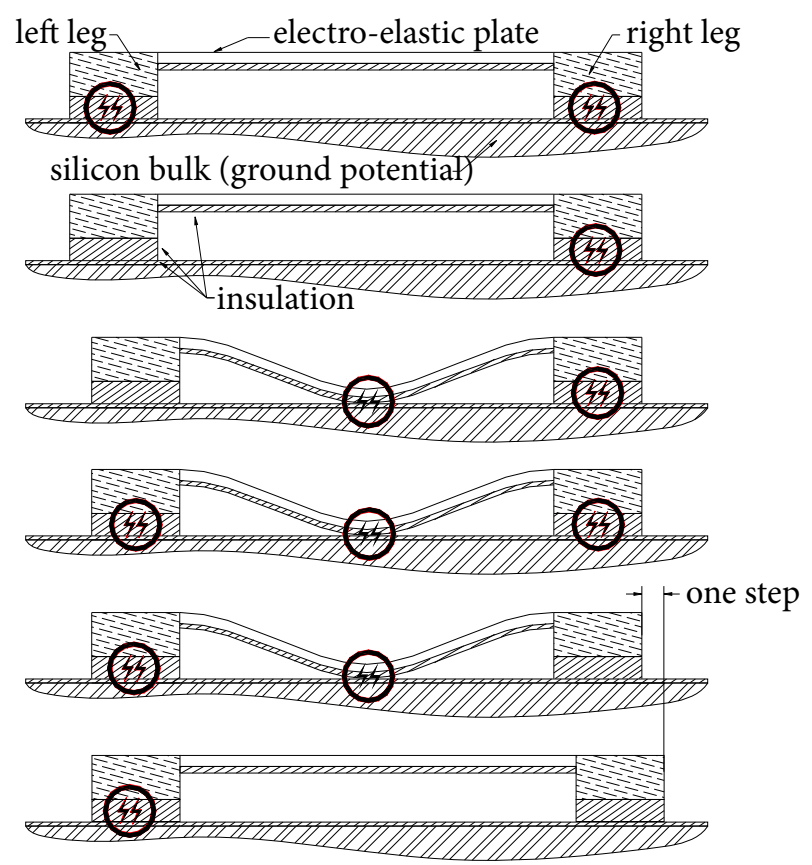

FIGURE 2.8 - Step sequence of the shuffle motor by Patrascu et al. (2007). The circles indicate that a voltage is applied on the corresponding leg or plate.

no way to compensate for it.

\subsubsection{Piezoelectric}

Piezoelectric actuation is commonly used for scanning probe microscopes, where there is ample space for the actuator. Piezoelectric material deforms when a voltage is applied, resulting in translational, rotational, or bending motion. An advantage of using piezo actuators is that their force is very large. However, piezo elements need to be quite large to provide the required displacement range for probe storage. But because of the large force, the areal efficiency can be high when mechanical stroke amplification is used to increase the displacement range of small piezo elements. The required voltage is reasonable in comparison to electrostatic actuators.

Several scanner designs using PZT actuators and stroke amplification are described by Zhang et al. (2003). The work continued and the improved design reaches $82 \mu \mathrm{m}$ at an applied voltage of $70 \mathrm{~V}$ (Faizul et al., 2009b). The scanner consists of a silicon MEMS displacement stage, to which PZT actuators have been attached manually (see Figure 2.9). The scan table is $9.5 \mathrm{~mm} \times 9.5 \mathrm{~mm}$ large. Although the fabrication of a micromachined stacked PZT actuator especially designed for the scanner is discussed, only measurements using commercially available larger PZT actuators are shown. The PZT actuators generate large forces $(\sim 3 \mathrm{~N})$ and their 


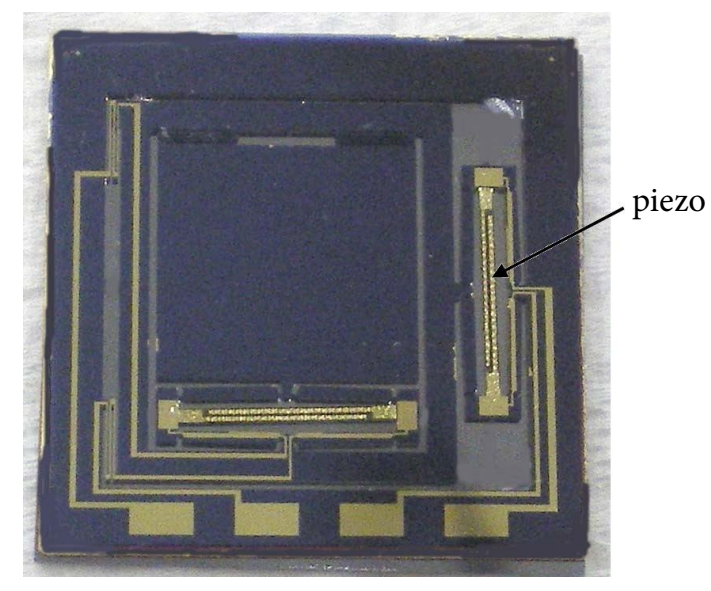

FIGURE 2.9 - Photograph of the silicon microstage by Faizul et al. (2009a) to which PZT stacked actuators have been attached. The total device is $2 \times 2 \mathrm{~cm}^{2}$ large.

small displacement is mechanically amplified 20 times to move the scan table. The maximum force on the scan table of $150 \mathrm{mN}$ is very large in comparison to other scanners discussed in this chapter. The displacement versus voltage measurements show hysteresis, but this may be reduced by using charge control instead of voltage control (Comstock, 1981). It will be interesting to see if the stiff springs provide a passive shock-resistance comparable to the shock resistance of IBM's mass-balanced electrodynamic scanner. The fabrication complexity of attaching the PZT actuators on the silicon stage is comparable to glueing of permanent magnets onto the electrodynamic scanner by IBM shown in Figure 2.1(c).

\subsection{Sensors and control}

Many MEMS displacement sensor types are suitable for probe storage systems (Bell et al., 2005). One of the challenges in a probe storage system is the need for nanometre resolution over a displacement range of $100 \mu \mathrm{m}$. To improve the positioning accuracy, a medium-derived position error signal can be used (Min and Hong, 2005; Sebastian et al., 2008). Several probe fields are designated as servo fields and are written by the device itself with special patterns; these servo fields are then used to obtain very precise information about the position of the probe array relative to the bit track.

The probe data storage prototype by IBM features a differential thermal displacement sensor, measuring the change in resistance due to the temperature change when the overlap between heater and scan table changes (Lantz et al., 2005). It provides $2.1 \mathrm{~nm}$ resolution (10 kHz bandwidth) at $10 \mathrm{~mW}$ power, and a maximum resolution of $0.49 \mathrm{~nm}$ at $32 \mathrm{~mW}$. Less than $6 \mathrm{~nm}$ of drift over 100 minutes was measured. The sensitivity strongly depends on the input power: $S \propto P^{1.6}$. The required 
power of a thermal sensor makes it less attractive for use in a mobile device. The sensor described by Lantz et al. (2005) is fabricated on a separate silicon wafer that is later bonded to the scan table. Recently, Krijnen et al. (2010) fabricated both thermal displacement sensors and an electrostatic comb-drive actuated scan table in the device layer of a silicon-on-insulator wafer using only one mask.

Capacitive sensors using comb drives are an attractive solution when comb drives are already used for actuation. An advantage is the lower energy consumption compared with thermal sensing. Comb drives are used as position sensors in commercial accelerometers, for example in air bag sensors and in the Nintendo Wii remote controller. An early example of a positioning system using comb drives for actuation and sensing is given by Cheung et al. (1996). Pang et al. (2009) from DSI in Singapore propose to use the same comb drive for actuation and sensing, however no measurement results are shown. Kuijpers (2004) from the University of Twente has studied an incremental long-range capacitive displacement sensor based on the capacitance change between two periodic structures, and obtained $10 \mathrm{~nm}$ resolution with, in principle, unlimited displacement range. The output of the sensor is periodic, and extra circuitry is used to count how many cycles have been measured. Unfortunately, the sensor has a low bandwidth of $5 \mathrm{~Hz}$, due to the delicate capacitive sensing circuitry (Kuijpers et al., 2006).

For the same reason a comb drive can be used as actuator and sensor, the electrode patterns in a dipole surface drive can also be used for sensing the position. Such a fine line capacitive position sensor integrated with a dipole surface drive is described by Hartwell et al. (2004). The resolution is $0.03 \mathrm{~nm} / \sqrt{\mathrm{Hz}}$. Lee et al. (2009) from Seagate use a similar capacitive sensor for their electrodynamic scanner. Like the incremental capacitive sensor by Kuijpers et al. (2006), the output signal of both sensors is an ambiguous measure of the position, requiring extra circuitry that keeps count of the number of cycles that have been measured. For the dipole surface drive that (Hartwell et al., 2004) used, Agarwal et al. (2006) describe a capacitive sensor with large plates with a resolution of $0.03 \mathrm{~nm} / \sqrt{\mathrm{Hz}}$. Because of the large plates, the output signal is no longer periodic and the output signal is a direct measure of the displacement.

IBM has performed extensive research on the control of their electrodynamic actuator (Pantazi et al., 2004, 2007; Sebastian et al., 2005, 2006, 2008). Different controller architectures have been investigated in order to optimize the seek time and the track-follow performance. Using $200 \mathrm{~mA}$ as maximum current for the electrodynamic scanner, the fastest seek time for $50 \mu \mathrm{m}$ movement is on the order of $1.6 \mathrm{~ms}$ (Pantazi et al., 2007).

Open-loop and closed-loop control for a shuffle motor was investigated by $\mathrm{Pa}$ trascu et al. (2007); unfortunately only a microscope with camera was available for position measurement, severely limiting the control bandwidth. The actuator position could be measured every $33 \mathrm{~ms}$ with $10 \mathrm{~nm}$ accuracy. A state-machine controller was designed, to generate the correct voltage sequences for making a small or large step in positive or negative direction or standing still. If the measured positioning error is non-zero, the state machine generates correction signals every $33 \mathrm{~ms}$ in the form of a number of steps into one direction. Open-loop control re- 
sulted in a maximum positioning error of $140 \mathrm{~nm}$. Closed-loop control was shown to work, however an integrated sensor with much larger bandwidth than the video setup is needed to obtain a fair assessment.

\subsection{Conclusion}

Important requirements for scanners for probe data storage a displacement range of $\pm 50 \mu \mathrm{m}$ with a nanometre resolution in two degrees-of-freedom, a large scan table such that many probes can be used in parallel, and an access time around $1 \mathrm{~ms}$. For mobile applications, shock-resistance, a low energy consumption and a small physical size are also required.

It is not yet clear which actuation type is most suited for probe data storage. The scanners for probe data storage that have been found in the literature, use several different actuator types; all designs use MEMS technology. MEMS technology makes it is possible to obtain a relatively large in-plane motion range within a small volume. The most prominent choices for which actuator type to use, are electrodynamic actuators or electrostatic comb drives. The only reported probedata storage prototype has been made by IBM, and uses electrodynamic actuators. The generated force is generally not large enough for the required shock-resistance, however, this can be remedied by applying a mass-balancing scheme employed in the scanner design by IBM. The use of comb drives results in a relatively straightforward fabrication process, however, the required footprint is large to obtain enough force at a reasonable driving voltage. Although electrostatic stepper motors, the dipole surface drive and the shuffle drive, offer potentially very high forces at low voltages, their use is complicated, among others, by a relatively complex fabrication process and control circuit. The scanner design using piezoelectric actuators with mechanical amplification looks promising, but the design is relatively young and not much information is available for a good judgement. 



\section{Chapter 3}

\section{A mass-balanced electrostatic $x / y$-scanner}

One of the promising candidates for future storage applications is parallel-probe data storage, in which many atomic-force-microscope-like probes are operated in parallel to read and write data at a high data rate and a high data density (> 1 Tbit in $^{-2}$; Cannara et al., 2008; Pantazi et al., 2008). Below the array of probes, the storage medium is moved in $x$ and $y$ directions by a positioning system (the scanner) with nanometre accuracy. To fully utilize the storage medium, the scanner's displacement range should be larger than the distance between the probes. As the probe density is on the order of 100 cantilevers per square millimetre (Despont et al., 2004), the scan range has to be relatively large, on the order of $\pm 50 \mu \mathrm{m}$.

Shock resistance and low power consumption are important requirements for mobile probe-storage applications (Lantz et al., 2007). Previously, a shock-resistant scanner design using electrodynamic actuation was reported by Lantz et al. (2007). Here we explore electrostatic actuation with shaped comb-drive fingers as an alternative to the electrodynamic actuation used by Lantz et al., because, in principle, comb drives do not require power to maintain a non-equilibrium position.

The total power consumption of the electrodynamic scanner by Lantz et al. is dominated by track follow rather than seek operations because seek operations are much shorter. When reading the outermost track ( $50 \mu \mathrm{m}$ displacement), the electrodynamic scanner's $x$-axis consumes $20 \mathrm{~mW}$ on average and the $y$-axis $60 \mathrm{~mW}$ continuously, which is almost a third of the SD flash memory card power budget of $300 \mathrm{~mW}$. The power consumption can be reduced by optimizing the scanner stiffness and the actuator design, however, power is always required for an electrodynamic actuator to maintain a non-equilibrium position. An advantage of electrostatic comb-drives compared to electrodynamic actuation is that, neglecting

This chapter is based on J. B. C. Engelen, H. E. Rothuizen, U. Drechsler, R. Stutz, M. Despont, L. Abelmann, and M. A. Lantz, "A mass-balanced through-wafer electrostatic $x / y$-scanner for probe data storage”, Microelectron. Eng., 86, pp. 1230-1233, 2009, and J. B. C. Engelen, M. A. Lantz, H. E. Rothuizen, L. Abelmann and M. C. Elwenspoek, "Improved performance of large stroke comb-drive actuators by using a stepped finger shape", presented at TRANSDUCERS 2009, pp. 1762-1765, 2009. 
leakage, no energy is consumed to maintain the actuator position. The comb drive's force is caused by charge instead of current flow; once a comb drive is put at a certain position by charging the combs, no more energy has to be supplied to maintain its position. Thus during track follow, when one axis is stationary and the other is moving slowly, the energy consumption of comb drives is potentially much lower compared to electrodynamic actuation. A disadvantage of using comb drives is the high voltage required for operation, typically many times the standard voltage available in a mobile storage device. DC/DC conversion is used commonly in mobile devices ( $12 \mathrm{~V}$ for Flash memory and $28 \mathrm{~V}$ for an LCD display); and conversion from $3 \mathrm{~V}$ to $380 \mathrm{~V}$ has been demonstrated for MEMS applications (Saheb et al., 2007). In our design, we have assumed that $150 \mathrm{~V}$ is a reasonable upper limit.

\subsection{Design}

An electrostatic comb-drive actuator (Tang et al., 1989) uses the attractive force between opposite electrical charges to generate a force. Two interdigitated electrodes, a fixed stator and a moving translator, are charged oppositely by applying a voltage across the gap between the electrodes. The magnitude of the comb drive force $F_{\text {comb }}$ can be found using the principle of virtual work (Johnson and Warne, 1995),

$$
F_{\text {comb }}=\frac{1}{2} \frac{\partial C}{\partial x} V^{2}
$$

where $x$ is the displacement, $C$ the capacitance between the electrodes, and $V$ the applied voltage. The generated force is always attractive, proportional to the square of the applied voltage. This means that two comb drives are required to actuate in both positive and negative $x$-directions.

In order to achieve shock resistance in the planar $x$ and $y$ directions while being compliant for actuation, we adopt the mass-balancing concept by Lantz et al. (2007). The electrodynamic actuators are replaced by electrostatic comb-drives, keeping the rest of the design intact (see Figure 3.1). To obtain a comparable force the total area is increased by $34 \%$. To move the scan table $50 \mu \mathrm{m}$ in both positive and negative $x$ and $y$ directions, four comb drives, consisting of a fixed stator and a moving translator, are used. The comb drives are coupled to the scan table through pivoting elements, such that the scan table and translator move in opposite directions. When the pivot ratio matches the inverse ratio of the scan table and translator masses, the equally directed inertial forces of a linear shock are cancelled, providing enhanced linear shock resistance.

The scan table is supported by a C-bracket suspended to the fixed outer frame, to decouple movement in the $x$ and $y$ directions. In the $y$ direction, the scan table and the C-bracket move as one; in the $x$ direction, only the scan table moves. This means that the moving mass in the $x$ direction is smaller than in the $y$ direction. Because the comb-drive translator masses are equal to the scan table plus C-bracket mass, a 1:1 pivot ratio is used in the $y$ direction. In the $x$ direction, a 1:1.36 pivot 


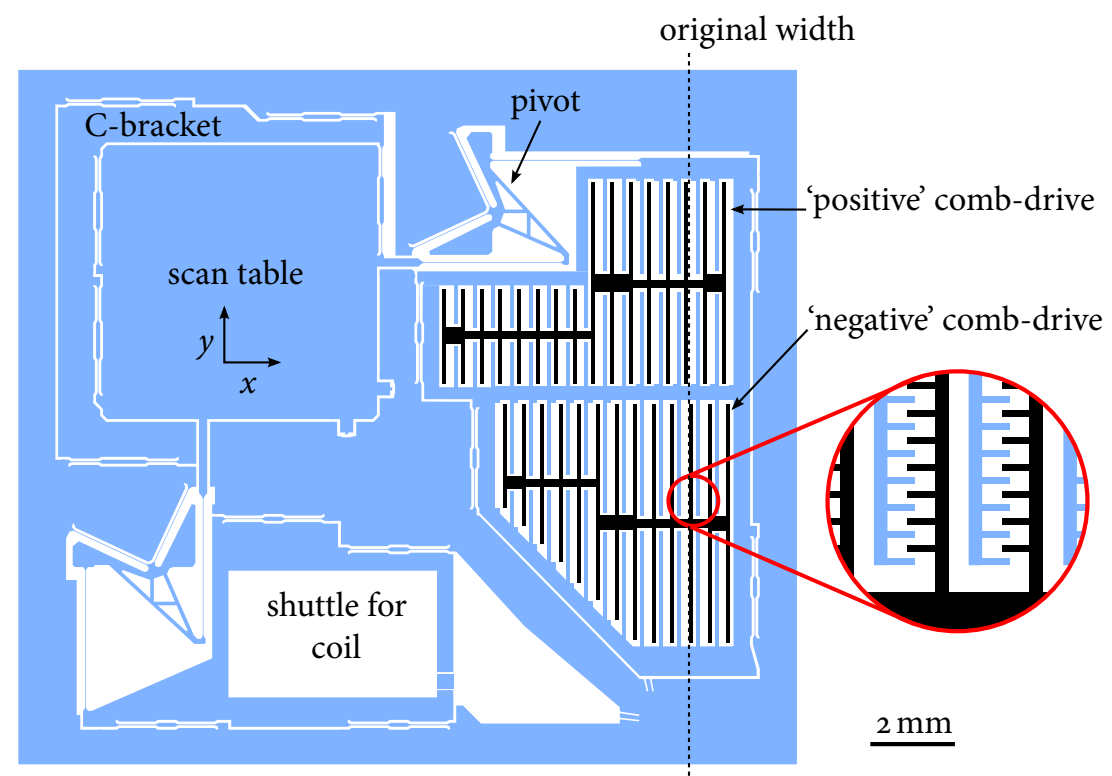

FIGURE 3.1 - The electrodynamic actuators (coils and permanent magnets) of the original scanner design are replaced by electrostatic comb-drives. Here, a design snapshot is shown, where the actuation for the $x$-axis is replaced. Two comb drives are needed to obtain negative and positive displacements; the fixed comb-drive stators are coloured black. The final design contains comb drives for both directions. The dashed line indicates the width of the original design with two electrodynamic actuators; the area of the final design is $34 \%$ larger than the original.

ratio is used (i.e. the scan table moves a factor 1.36 more than the corresponding translator).

A large out-of-plane stiffness for passive shock rejection in the $z$ direction is achieved by etching the device, including the spring suspension, through the full thickness of the wafer $(400 \mu \mathrm{m})$. This large etch depth necessitates the use of a relatively large gap between fingers $(25 \mu \mathrm{m}$, see $\$ 3.2)$, resulting in a reduced actuation force. To increase the generated force without decreasing the minimum etch trench width, two comb finger shapes are investigated: a tapered shape (Mohr et al., 1992; Rosa et al., 1998) and a new 'stepped' shape, both shown in Figure 3.2.

To perform seek operations or compensate for residual shock forces, the actuation force at any given position $x$ must exceed the suspension spring restoring force. The force available for these control operations is

$$
\begin{aligned}
& F_{\text {avail }}(x)=F_{\text {comb }}\left(x, V_{\max }\right)-k x, \\
& F_{\text {avail }}(x)=\frac{1}{2} N \frac{\partial C}{\partial x} V_{\text {max }}^{2}-k x,
\end{aligned}
$$



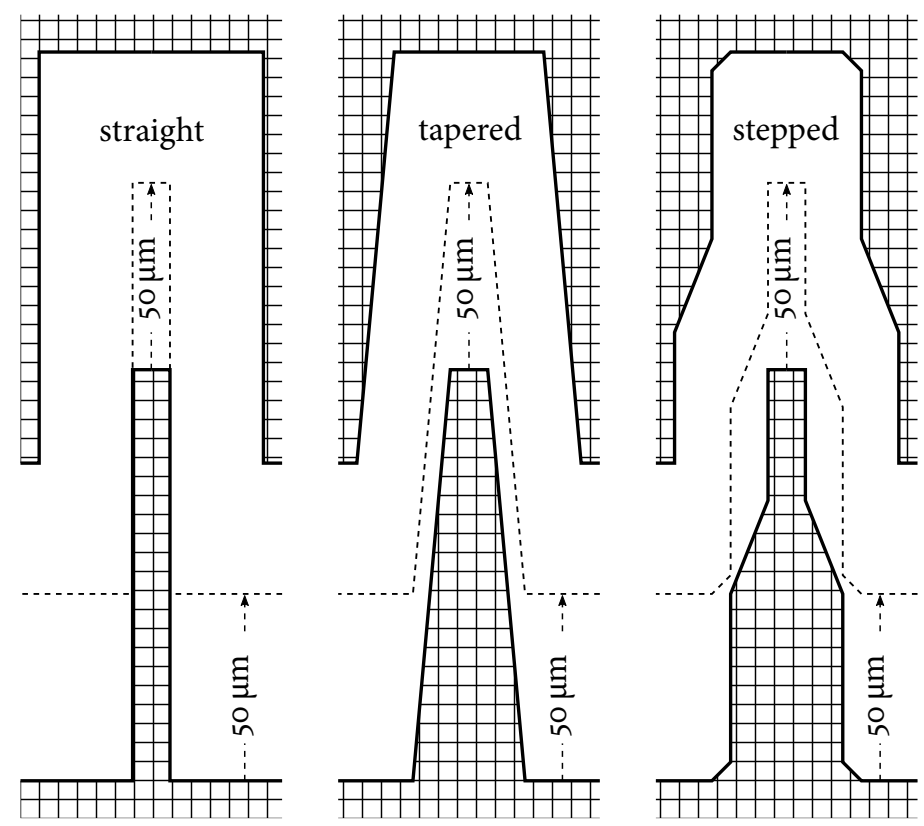

FIGURE 3.2 - The three investigated finger shapes, drawn to scale. The new stepped finger shape is shown on the right. The dashed outline shows the geometry at $50 \mu \mathrm{m}$ displacement.

where $F_{\text {comb }}$ is the actuation force generated by the comb drive, $V_{\max }$ the maximum available voltage, $k$ the spring constant of the suspension, $x$ the displacement, $N$ the number of finger pairs, and $C$ the capacitance of one finger pair. This available force $F_{\text {avail }}$ should be greater than zero within the operating range to enable control operations and ideally should be as large as possible. Figure 3.7 shows a simulation result for the available force curve for a normal straight finger shape, from which it can be seen that the force available at large positive displacements needs to be increased in order to extend the range of achievable displacement and to provide a positive force margin for control operations.

As will be discussed in the next chapter, the use of 'gap-narrowing' finger shapes (i.e. shapes that narrow the gap between fingers at larger displacements) increase the force for large displacements and is most suited for our application. For straight fingers, the necessary change in capacitance $\frac{\partial C}{\partial x}$ is caused by increasing finger overlap; for gap-narrowing finger shapes, an extra term due to the decreasing gap distance between fingers adds to the capacitance change, hence an increase in force. In this chapter, we will discuss the use of tapered and 'stepped' comb-drive fingers. The gap-narrowing effect of the tapered and stepped shapes can be seen in Figure 3.2, when comparing the geometries at $\mathrm{o} \mu \mathrm{m}$ and $50 \mu \mathrm{m}$ displacement. The next chapter will discuss the optimal finger shape for maximum shock-resistance. 


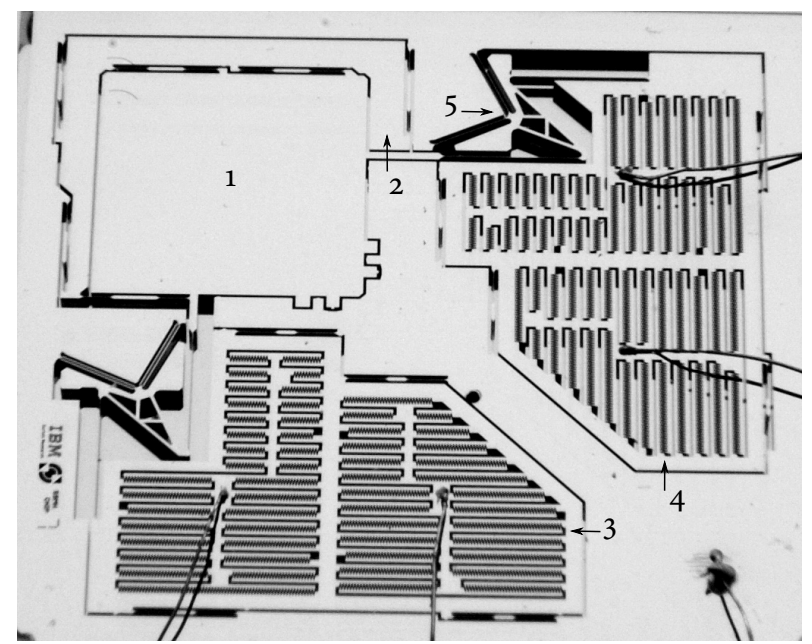

Figure 3.3 - Photograph of a fabricated device $(2 \mathrm{~cm} \times 2 \mathrm{~cm})$. 1: scan table, 2: C-bracket, 3: $x$ comb drives, 4: $y$ comb drives, 5: pivoting element. The wires are electrical connections to the isolated stators and to ground the moving parts of the device (bottom right).

\subsection{Fabrication}

The scanners are fabricated from a $400 \mu \mathrm{m}$ thick, highly-doped single-crystal silicon wafer, using deep reactive-ion etching and a layer of aluminium as an etch stop. A photograph of a fabricated scanner is shown in Figure 3.3. All the mechanical parts including the comb drives are etched through the full thickness of the wafer. For a uniform etch load, the whole mask design is defined with a fixed etch trench width of $20 \mu \mathrm{m}$ and a minimum structure width of $15 \mu \mathrm{m}$. The resulting trench width after etching is $25 \mu \mathrm{m}$ and the minimum structure width is $10 \mu \mathrm{m}$. Large openings are defined by a $20 \mu \mathrm{m}$ trench along their periphery, leaving a centre piece that drops out when the aluminium etch stop layer is removed. With these rules, the minimum finger width is $10 \mu \mathrm{m}$ and the gap between comb-drive fingers after etching is $25 \mu \mathrm{m}$. A larger minimum width constraint of $40 \mu \mathrm{m}$ is used at the base of the fingers (where the etch area is enclosed on three sides, instead of two sides for a continuous trench), to obtain a more homogeneous etch rate across the geometry. These openings within the comb drives have no drop-out pieces. The finger shape is preserved well through the thickness of the wafer, as can be seen in Figure 3.4(a).

The comb drive stators are completely surrounded by the translators; several break-out pieces connect the stators mechanically to the translators to prevent the stators from falling out at the end of the etch process (see Figure 3.4(b)). After etching, individual scanner chips are separated from the wafer and glued to base plates. A base plate features several arrays of supports on which glue is applied; the clearance between moving parts and base plate is about $100 \mu \mathrm{m}$. Subsequently, the 


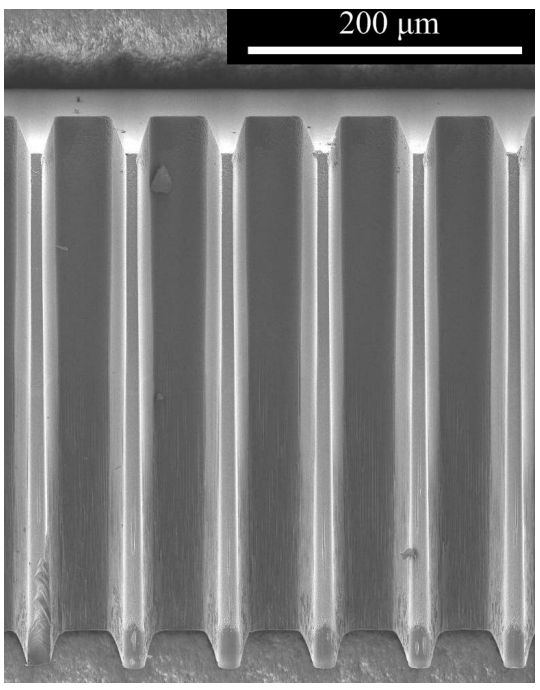

(a) Scanning electron micrograph of the tapered comb drive fingers (the wafer front side is seen at the top). The shape is preserved within several micrometres; there is an increase in finger width toward the back of the wafer.

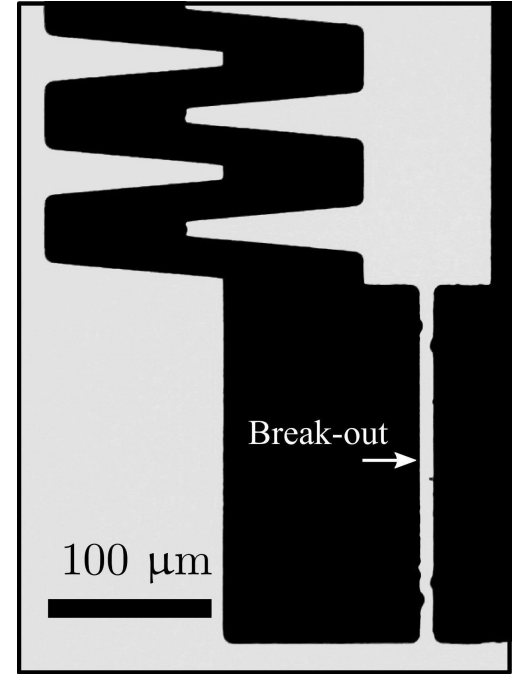

(b) Optical micrograph of the front side, showing the break-out piece between stator and translator. The break-out piece is designed with small indents on both ends for easier breaking.

FIGURE 3.4 - Images of the fabricated scanner with tapered fingers.

break-out pieces are removed to provide electrical insulation between the stator and the translator of the comb drives. Finally, small electrical leads are connected to the device using conducting epoxy.

\subsection{Results}

The fabricated devices are analysed using stroboscopic video microscopy measurements. For the scanner with tapered fingers, a UMECH motion analyzer was used (UMECH MEMS Motion Analyzer, Umech Technologies); for the scanner with stepped fingers, we used a Polytec MSA40o and its Planar Motion Analyzer software (Planar Motion Analyzer 2.5, Polytec MSA40o, Polytec GmbH).

As can be seen from the measured frequency response and phase shift of the scanner with tapered fingers, shown in Figure 3.5, each axis behaves like a secondorder mass-spring-damper system. The measured resonance frequencies for the $x$ and $y$ axes are $147 \mathrm{~Hz}$ and $133 \mathrm{~Hz}$, respectively, in good agreement with the values of $148 \mathrm{~Hz}$ and $130 \mathrm{~Hz}$ predicted by mechanical FE simulations. The scanner with stepped fingers shows similar results.

Figure 3.6 shows the displacement of the slow axis versus voltage curve measurements for the tapered and stepped finger shape devices. The measured reso- 


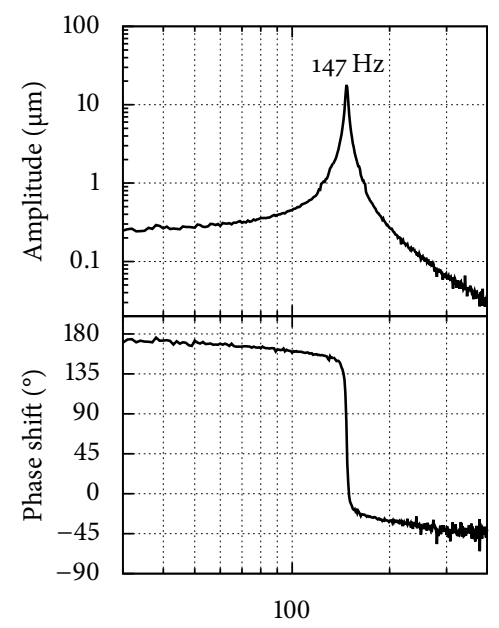

Frequency $(\mathrm{Hz})$

(a) Fast axis

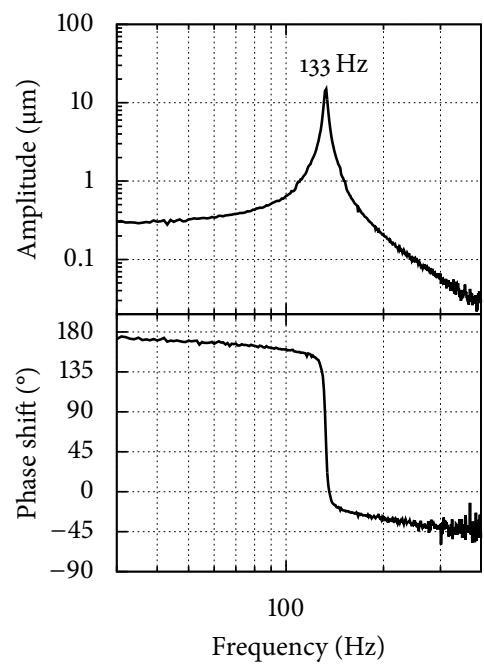

(b) Slow axis

FIGURE 3.5 - Bode plots for both axes of the scanner with tapered fingers. Both axes are second-order systems to a good approximation.

nance frequencies for the corresponding axes of the tapered and stepped designs were $133 \mathrm{~Hz}$ and $147 \mathrm{~Hz}$, respectively. Using these frequencies and measured actuator and scan table masses, mechanical finite-element simulations predict effective spring constants of $60 \mathrm{~N} \mathrm{~m}^{-1}$ and $72 \mathrm{~N} \mathrm{~m}^{-1}$, for the tapered and stepped designs respectively, although the suspensions should have identical stiffness by design. The difference stems from variations in the etch profiles actually obtained. At $120 \mathrm{~V}$, displacements of $38 \mu \mathrm{m}$ and $48 \mu \mathrm{m}$ were measured for the tapered and stepped fingers, respectively (Figure 3.6), corresponding to $2.3 \mathrm{mN}$ and $3.5 \mathrm{mN}$, respectively, using the calculated stiffness values.

Figure 3.7 shows 2D electrostatic finite-element results for the available force of the three finger shapes. The equilibrium voltage $V_{\text {eq }}$ measurements in Figure 3.6 are used to calculate the available force; combining equation (3.2) and the equilibrium condition

$$
\frac{1}{2} N \frac{\partial C}{\partial x} V_{\mathrm{eq}}^{2}=k x
$$




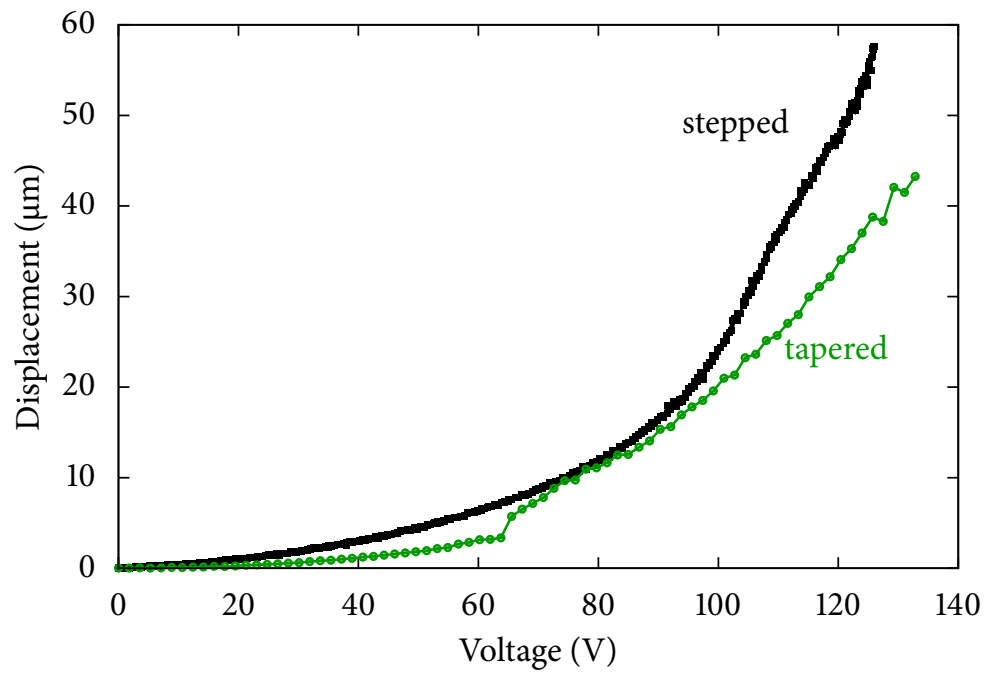

FIGURE 3.6 - Displacement measurements on a device with tapered and a device with stepped fingers. The feature in the tapered finger curve at $65 \mathrm{~V}$ is a measurement error caused by video processing.

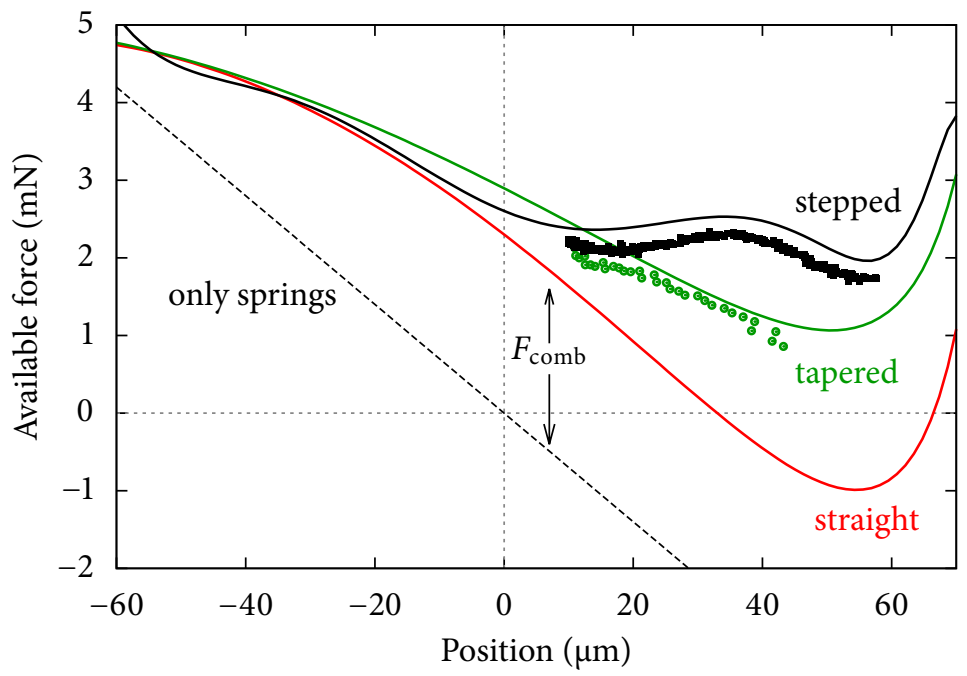

FIGURE 3.7 - The maximum available force $\left(=F_{\text {comb }}-k x\right)$ in the positive direction as calculated by $2 D$ electrostatic finite-element simulations (solid lines, polynomial fit, $V_{\text {max }}=150 \mathrm{~V} ; k=72 \mathrm{~N} \mathrm{~m}^{-1} ; 731$ finger pairs) and as calculated from the measurements in Figure 3.6 (points). The dashed curve shows the force from the suspension springs only. 
we obtain

$$
F_{\text {avail }}(x)=k x \frac{V_{\mathrm{max}}^{2}}{V_{\mathrm{eq}}^{2}}-k x .
$$

The calculation results are shown as points in Figure 3.7; the difference with the finite-element result is explained by a slightly larger obtained finger gap size.

The device using stepped fingers reaches a $25 \%$ larger displacement than the device using tapered fingers, in spite of the $20 \%$ stiffer spring suspension of the stepped shape device. Stepped fingers provide the highest force and their available force curve is almost flat for positive displacements, approximating an optimal solution where the minimum in the available force curve is as high as possible (see Chapter 4). During the design of the stepped shape, finite-element calculations were used to inspect the raised part of the available force curve (around $35 \mu \mathrm{m}$ ), because a too high local maximum will lead to unstable displacements at the start of the raised part (see \$4.1.2). Assuming a maximum voltage of $150 \mathrm{~V}$, the device using the stepped shape has at least $1.8 \mathrm{mN}$ force available for control operations for the full $\pm 50 \mu \mathrm{m}$ range.

Interestingly, the available force is largest in the region where the fingers are disengaged and have no overlap $(x<-25 \mu \mathrm{m})$; the comb drive force decreases, but the spring force increases and because the displacement is negative, it adds to the available force in the positive direction. Contrary to what one might expect, the minimum of the available force curve lies not in the region where the fingers are disengaged and $F_{\text {comb }}$ is small, but in the region where $F_{\text {comb }}$ is large and where the comb drive is close to instability (snap-in).

\subsection{Conclusion}

We have designed and successfully fabricated a mass-balanced vibration resistant $x / y$-scanner that uses electrostatic actuation. To provide passive shock rejection in the $z$ direction perpendicular to movement, the $x / y$-scanner is fabricated from the full thickness of the wafer $(400 \mu \mathrm{m})$. In order to etch comb drives through this thickness, the gap between comb drive fingers was relatively large $(25 \mu \mathrm{m})$.

An important conclusion from the available force calculations is that, although the fingers are disengaged for large negative displacements, the available force in the positive direction is still large in this region due to the restoring spring force; the minimum available force in the positive direction is found at large positive displacements. This means that, in terms of available force, it is not necessary to have comb fingers overlap throughout the whole actuation range.

The comb drive force was increased by using gap-narrowing finger shapes. Measurements showed that the displacement at $120 \mathrm{~V}$ using stepped fingers is $26 \%$ more than using tapered fingers, despite the spring suspension of the stepped shape device being $20 \%$ stiffer than the spring stiffness of the device with tapered fingers. Moreover, the available force for control operations is larger for the stepped shape compared to the straight and tapered finger shapes. The available force curve of the stepped shape approximates an optimal solution where the available force curve 
is flat and its minimum is as high as possible. To obtain a force and displacement range comparable with the original electrodynamic scanner, the footprint needed to be increased by $34 \%$. However, the thickness of the scanner is reduced, because the electrodynamic scanner requires permanent magnets above or below the scanner. Combined, the electrostatic scanner is more volume efficient.

\subsection{Acknowledgements}

The work presented in this chapter was primarily done at IBM Zürich Research Laboratory, Rüschlikon, Switzerland. I am very grateful I was granted this opportunity, and want to thank the people at IBM for a very nice stay. The design of the comb drives was done together with Mark Lantz and Hugo Rothuizen. Richard Stutz drew the masks, Ute Drechsler etched the structures, and Martin Witzig helped assembling the scanners. Back at the University of Twente, Remco Sanders helped operating the Polytec. 


\section{Chapter 4}

\section{Optimized comb-drive finger shape for shock-resistant actuation}

Electrostatic comb drives are frequently used for actuation in MEMS, mainly because fabrication is relatively simple compared to other actuator types, such as piezo or electromagnetic actuators. However, the obtainable maximum force of standard comb-drive actuators (with straight fingers) is relatively low. When using comb drives in a $x / y$-nanopositioner, or scanner, for a probe data-storage system (Pantazi et al., 2008), this low force is an issue, as described in the previous chapter. The scanner (Figure 4.1) has to move a scan table with the storage medium over a large range (typically $100 \mu \mathrm{m}$ ), and has to function in the presence of external shocks and vibrations. Therefore, shock and vibration resistance of the scanner is important, especially for operation in mobile devices. The spring suspension can be made stiff for rotations and movement in the $z$ direction for passive shock resistance, however since movement along the $x$ and $y$ axes is desired, the spring stiffness in the $x$ and $y$ directions is necessarily low to enable actuation. External shocks and vibrations result in inertial forces on the scan table, which can be counterbalanced by the inertial forces on the actuator, via a mass-balancing scheme (Lantz et al., 2007). By introducing a pivot point and oppositely linking the scan table and actuator mass, the movement of the scan table is always opposite to the actuator mass (e.g. when the actuator moves to the left, the scan table moves to the right). If the masses of the scan table and actuator are matched, the initial forces on them will be equal in size and direction, and will cancel each other through this opposite linking. Nevertheless, a large actuator force is required to compensate residual inertial forces due to imperfect mass matching and rotational accelerations about the pivot point.

To compensate shock forces, the actuation force at any given displacement $x$ must exceed the suspension springs restoring force. The available force $F_{\text {avail }}$ is equal to the maximum actuator force $F_{\text {act,max }}$ minus the suspension springs

This chapter is based on J. B. C. Engelen, L. Abelmann, and M. C. Elwenspoek, "Optimized combdrive finger shape for shock-resistant actuation”, J. Micromech. Microeng., 20, 105003, 2010. 
force $k x$,

$$
F_{\text {avail }}(x)=F_{\text {act,max }}(x)-k x,
$$

where $k$ indicates the suspension spring stiffness. The minimum value of the available force throughout the displacement range is paramount, as it determines the maximum shock force that can be compensated for at any displacement. 1996)

The force generated by a voltage controlled comb drive equals (Legtenberg et al.,

$$
F_{\text {comb }}(V, x)=\frac{1}{2} N \frac{\partial C}{\partial x} V^{2},
$$

where $N$ is the number of unit cells (i.e. the number of finger pairs), $\frac{\partial C}{\partial x}$ the change in capacitance between the finger pair in one unit cell, and $V$ the applied voltage. The standard comb-drive design features straight comb fingers, which have a constant $\frac{\partial C}{\partial x}$ and hence result in a constant maximum force independent of displacement. Therefore, the available force of a standard comb drive will be low at large displacements, where the spring force is maximum, severely limiting the shock resistance (see Figure 4.2(a)). From (4.1), we see that a maximum actuator force $F_{\text {act, max }}$ that increases with displacement is desired for shock-resistant actuation.

The force characteristic (force versus displacement) of a comb drive can be tailored by reducing the spring stiffness $k$, by modifying $\frac{\partial C}{\partial x}$. Because a minimum out-of-plane stiffness is required, reduction of $k$ is undesired, and provides only limited control of the force characteristic. An obvious way to increase the combdrive force is increasing the number of finger pairs, however this increases the footprint and mass of the scanner and is therefore unattractive. Obeying the minimum gap size limit of the fabrication process, tailoring $\frac{\partial C}{\partial x}$ can be done by changing the individual lengths of straight fingers, such that finger pairs engage at different displacements (Grade et al., 2003). However, when all finger pairs are engaged (maximum force), the number of finger pairs and gaps between fingers is the same as for a standard comb drive, and hence the maximum force does not increase. Modifying individual finger lengths decreases the force at small displacements and does not increase the force for large displacements. Effectively, changing the lengths of fingers only allows tailoring of the available force within the available force limits of the standard comb drive (indicated by the shaded region of Figure 4.2(a)).

More suited for our purpose is modifying $\frac{\partial C}{\partial x}$ by changing the shape of the comb fingers, e.g. tapering the fingers as in the previous chapter. Vertical shaping (Morgan and Ghodssi, 2008) is not desired because it does not increase the force and is not possible in most fabrication processes anyway. The previous chapter showed the application of comb drives with a stepped finger shape in a probe-storage scanner, leading to a strong increase in both maximum stroke and force. However, this stepped shape can result in an unstable region, requiring careful adjustment of the shape using finite-element simulations to ensure stability over the full operation range. We'll use the term 'snap-in' to denote instability in the direction of desired motion $x$, in contrast to 'side snap-in' that concerns unstable behaviour in the direction perpendicular to $x$. Side snap-in can occur for individual fingers (fingers 


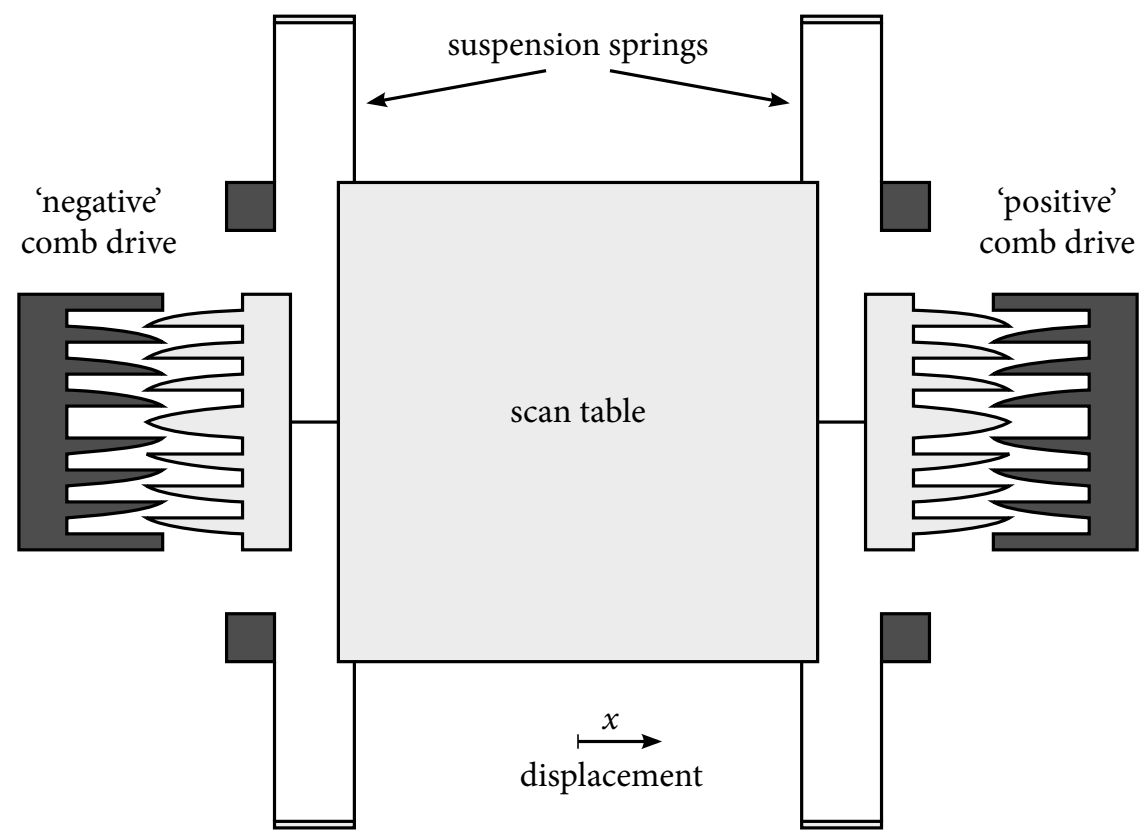

FIGURE 4.1 - Schematic illustration of a $1 D$ scanner with comb-drive actuators for positive and negative displacements. Light shaded areas are free to move, suspended by the springs; dark shaded areas are fixed.

bending towards each other) and for a whole comb drive (sideways bending of spring suspension).

Ye et al. (1998) reported a numerical optimization of finger shapes for several polynomial force characteristics. An analytical model for calculating the force characteristic of arbitrary finger shapes is described by Jensen et al. (2003). We will use this analytical model as starting point of our analysis. These works describe finger shapes that result in a linear dependence of the force on displacement, similar to the shape described in this work. However, the described finger shapes result in thick fingers, reducing the force per unit length. Moreover, the issue of the optimal force characteristic for shock-resistance and the analytical solution for the finger shape that provides this force characteristic remained unaddressed.

In the following, we describe a new finger shape with an increased maximum force at large displacements, leading to a larger shock resistance. We show that a constant available force throughout the comb drive's operating range is the optimal solution for shock resistance and large stroke, combining the highest available force with the largest side-stability (see Figure 4.2(b)). The analytical solution for the finger shape with constant available force is presented together with finite-element calculations and measurements on fabricated comb drives. 


\subsection{Theory}

This section discusses the optimal finger shape for use in scanners like the one shown in Figure 4.1. Two identical comb drives are used to displace the scan table in both positive and negative directions. This effectively doubles the range of obtainable displacements without increasing the maximum voltage. The scanner is symmetric for positive and negative displacements. Except when explicitly mentioned otherwise, it is assumed that the displacement $x$ is positive and the 'positive' comb drive is actuated.

\subsubsection{Analytical model}

The maximum force of a comb drive is obtained when applying the maximum voltage $V_{\max }$ of the available voltage source. The available force of a comb drive with suspension springs therefore equals

$$
F_{\text {avail }}(x)=F_{\text {comb }}\left(x, V_{\max }\right)-k x,
$$

Because the maximum force of a standard comb drive with straight fingers is constant when the fingers are engaged, the available force equals a straight downward curve, shown in Figure 4.2(a). As can be seen from this figure, the available force and therefore the range of forces that can be applied (the shaded area) depend strongly on the displacement. The shock resistance of the whole system is low because the available force at large displacements is low.

By modifying the shape of the fingers, it is possible to increase the available force beyond the shaded region of Figure 4.2(a). When the base of the fingers is thicker than the tip, the gap between fingers becomes smaller for increasing displacement, and the force increases. However, it is not possible to increase the available force at zero displacement $F_{\text {avail, } 0}$, because the minimum gap between the tips of the fingers is determined by the fabrication process' design rules; all finger shapes will result in the same $F_{\text {avail,0. }}$.

Jensen et al. (2003) describe an analytical model for calculating the force of a comb drive with arbitrary finger shapes. The model uses a parallel-plate approximation, giving accurate predictions for comb drive fingers with continuous shapes that are approximately parallel to each other. Limiting the design freedom by assuming that one of the fingers is straight (see Figure 4.3), results in an important simplification for the expression of the capacitance $C$ of one comb-drive unit cell,

$$
C(x)=2 \varepsilon_{0} h \int_{0}^{x_{0}+x} \frac{1}{g(s)} d s,
$$

\footnotetext{
${ }^{*}$ When tapered fingers are used, in some cases an increase in $F_{\text {avail, } 0}$ is seen, see Figure 3.7. However, in those cases, the distance between fingers in the design has decreased below the fabrication process' design rules.
} 


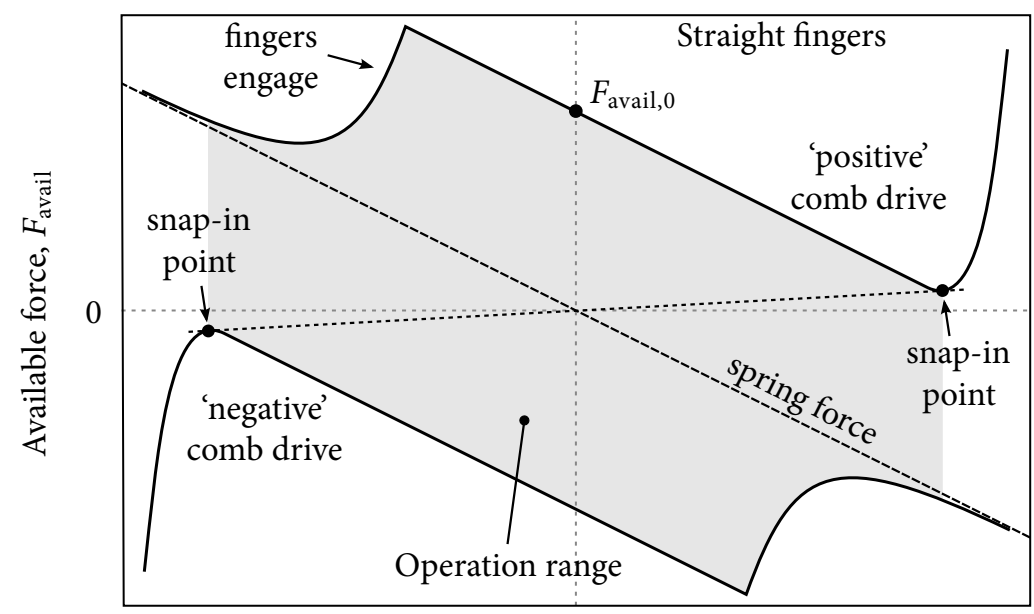

0

Displacement, $x$

(a) Sketch of the available-force curve for standard straight comb fingers. The snap-in point is determined from where a line from the origin is tangent to the available-force curve (this graphical method is described in more detail in \$4.1.2).

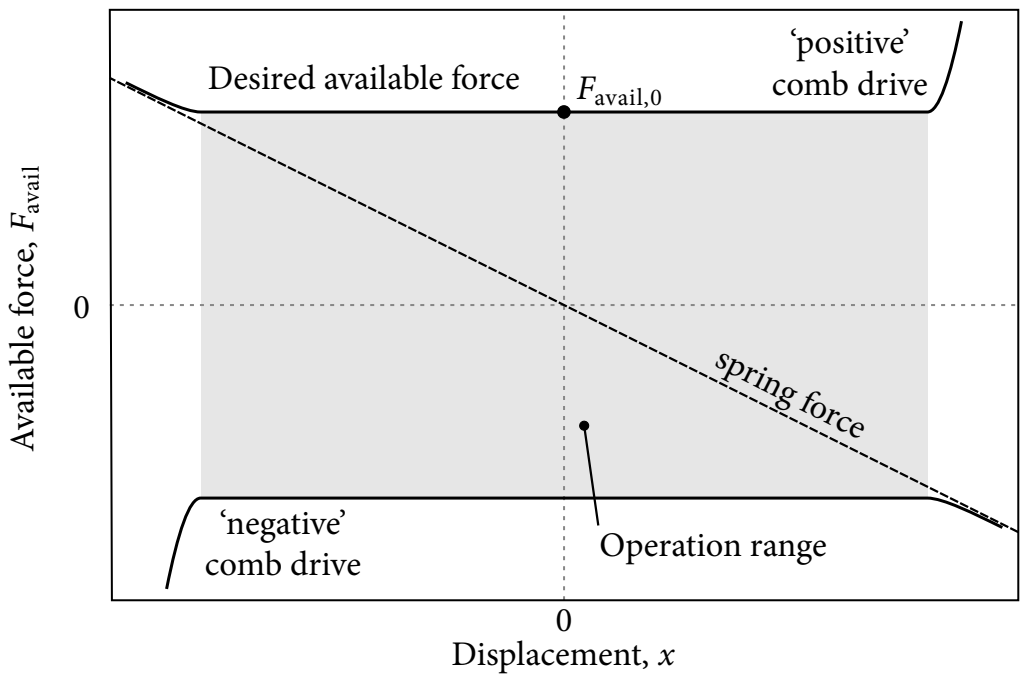

(b) Sketch of the optimal available-force curve for shock resistance. The range of forces that can be exerted on the scan table is independent of the displacement.

FIGURE 4.2 - Sketches of the available-force curve for straight fingers and the desired available-force curve. The range of forces that can be exerted on the scan table is indicated by the shaded areas. 


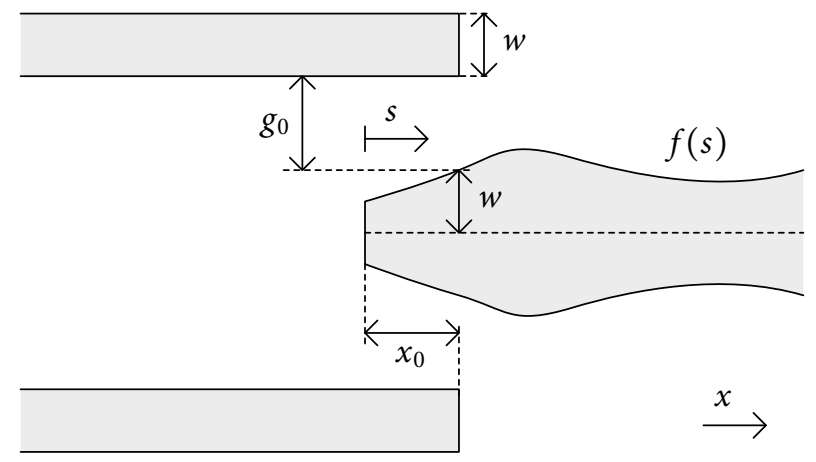

FIGURE 4.3 - The comb drive geometry with straight fingers on one side, and symmetrically shaped fingers on the other side. The initial overlap $(x=0)$ is $x_{0}$; at displacement $x$, the overlap equals $x_{0}+x$. Shape function $f(s)$ is defined from the centre of the shaped finger, where $s=0$ at the tip of the shaped finger. To have as many fingers per meter as possible, $w$ should be small. Because of the minimum gap size $g_{0}$ that can be fabricated, finger shape $f$ must be less or equal to $w$ for the part where the fingers initially overlap $\left(f(s) \leq w\right.$ for $\left.s \in\left[0, x_{0}\right]\right)$.

where $h$ is the height of the fingers, $x_{0}$ the initial overlap, $x$ the displacement, and $g(s)=g_{0}+w-f(s)$ the gap profile between fingers. Shape function $f(s)$ represents half the thickness of the finger at position $s$, and $w$ is equal to half the finger thickness at $s=x_{0}$. Normal straight fingers have finger shape $f(s)=w$. The force of the total comb drive then equals

$$
F_{\text {comb }}(x, V)=\frac{\varepsilon_{0} h N V^{2}}{g\left(x+x_{0}\right)},
$$

for a comb drive with $N$ finger pairs and an applied voltage $V$. Note that because one finger is straight, the force at displacement $x$ depends only on the gap width at the tip of the straight finger, $g\left(x+x_{0}\right)$, rather than the complete shape profile. Intuitively, this can be understood as follows: the force depends on the change in capacitance between the fingers, and the only change in capacitance occurs at the tip of the straight finger whose distance to the other finger varies with the displacement.

\subsubsection{Graphical determination of equilibrium stability}

Modifying the finger shape can lead to unstable behaviour. This section describes a method to quickly identify stability issues using an available force versus displacement graph.

When the scanner is in equilibrium at a positive displacement, the force of the 


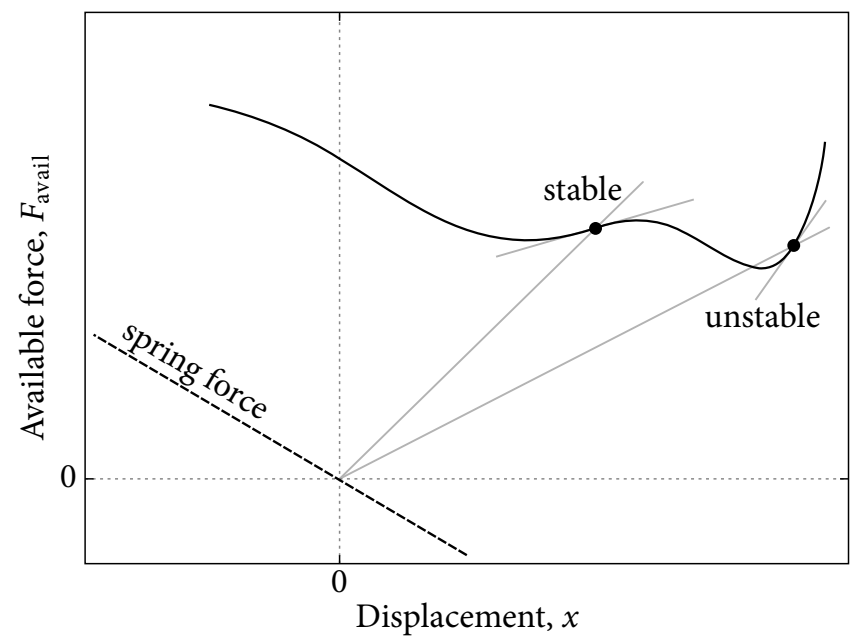

FIGURE 4.4 - Graphical determination of stability for two displacements of an arbitrary available-force curve, by drawing a line from the origin to a point on the available-force curve. When the derivative of this line is larger than the derivative of the curve, the point is stable, otherwise the point is unstable. Snap-in occurs at points where the line from the origin is tangent to the curve.

'positive' comb-drive and the spring force exactly counteract each other:

$$
\begin{aligned}
& -F_{\text {spring }}=F_{\mathrm{comb}}, \\
& k x=\frac{1}{2} N \frac{\partial C}{\partial x} V_{\mathrm{eq}}^{2},
\end{aligned}
$$

from which follows

$$
V_{\mathrm{eq}}= \pm \sqrt{\frac{2 k x}{N \frac{\partial C}{\partial x}}} .
$$

Because the comb-drive force is proportional to the square of the applied voltage, the sign of the applied voltage is irrelevant. To simplify the notation in the following discussion, we will assume a positive equilibrium voltage $V_{\mathrm{eq}}$.

To be able to control the displacement by a constant equilibrium voltage, the derivative of (4.6) should be larger than zero, from which we obtain constraints on $\frac{\partial C}{\partial x}$ for stable voltage control. Because $\sqrt{x}$ is a strictly increasing function, it is not relevant for the sign of the derivative of $(4.6) ; \frac{2 k}{N}$ is constant and positive, and is 
therefore irrelevant for the sign too. For stable voltage control,

$$
\begin{aligned}
\frac{\partial V_{\mathrm{eq}}}{\partial x}>0 \Rightarrow & \frac{\frac{\partial C}{\partial x}-x \frac{\partial^{2} C}{\partial x^{2}}}{\left(\frac{\partial C}{\partial x}\right)^{2}}>0, \\
& 1-\frac{\frac{\partial^{2} C}{\partial x^{2}}}{\frac{\partial C}{\partial x}} x>0 .
\end{aligned}
$$

Note that the spring stiffness $k$ does not influence the stability range of any comb finger shape.

An elegant graphical way of locating stability issues is found after rearranging (4.7), multiplying by $\frac{1}{2} V_{\max }^{2}$, and subtracting $k$ :

$$
\frac{1}{2} V_{\max }^{2} \frac{\partial^{2} C}{\partial x^{2}}-k<\frac{\frac{1}{2} V_{\max }^{2} \frac{\partial C}{\partial x}-k x}{x} .
$$

The left hand side of (4.8) is equal to the slope of the available force versus displacement curve, and the right hand side equals the slope of the line from the origin to a point on the available-force curve. When the line from the origin to a point on the curve is steeper than the derivative at that point, the comb drive can be held stable at that point at the equilibrium voltage given by (4.6). Figure 4.4 shows a particular available-force curve and the determination of equilibrium stability of two displacements. Snap-in (in $x$-direction) happens at the transition from stable to unstable points, and can be determined from where the line from the origin is tangent to the curve (shown in Figure 4.2(a)).

Because $F_{\text {avail, } 0}$ cannot be increased, it determines the maximum shock resistance that can be obtained. A constant available force $F_{\text {avail }}(x)=F_{\text {avail, } 0}$ throughout the comb drive's operating range, shown in Figure 4.2(b), is the optimal solution for shock resistance and large stroke, because it combines the highest shock resistance with the largest side-stability. If the available force would be increased more, equilibrium voltage control would be more difficult as the displacement would be more sensitive to changes in applied voltage. Moreover, sideways forces would increase due to the decreasing gap at the base of the fingers, requiring a stiffer spring suspension to prevent side-instability.

\subsubsection{Design of optimal shape}

From the graphical method to determine stability, we obtained that a constant available force is optimal for shock resistance. For a constant available force $F_{\text {avail }}(x)=$ $F_{\text {avail,0, }}$, the comb drive force should equal

$$
F_{\text {comb }}\left(x, V_{\max }\right)=k x+F_{\text {avail }, 0}
$$

where $F_{\text {avail, } 0}$ is determined by the initial gap size $g_{0}$ as dictated by the minimum etch trench width of the fabrication process,

$$
F_{\text {avail, } 0}=\frac{\varepsilon_{0} h N V_{\max }^{2}}{g_{0}} .
$$


Combining (4.2) and (4.9), we find the optimal capacitance as a function of displacement for the unit cell,

$$
\begin{aligned}
\frac{1}{2} N \frac{\partial C}{\partial x} V_{\max }^{2} & =k x+F_{\text {avail }, 0} \\
C(x) & =\frac{k x^{2}}{N V_{\max }^{2}}+\frac{2 F_{\text {avail }, 0} x}{N V_{\max }^{2}}+C(0) .
\end{aligned}
$$

When $k=0,(4.12)$ equals the capacitance of the standard straight fingers unit cell (Legtenberg et al., 1996), with a different value for $C(0)$. Combining (4.6) and (4.11), the equilibrium voltage of a comb drive with this optimal capacitance is obtained,

$$
V_{\text {eq,optim }}= \pm \sqrt{\frac{k x V_{\max }^{2}}{k x+F_{\text {avail }, 0}}} .
$$

The new shape does not remove the non-linearity of the comb drive.

Combining (4.4) and (4.9), we can calculate the gap profile that has the optimal force characteristic and we obtain the solution for the optimal shock-resistant finger shape,

$$
f(s)=w+g_{0}-\frac{\varepsilon_{0} h N V_{\max }^{2}}{\left(s-x_{0}\right) k+F_{\text {avail, } 0}},
$$

where $s$ indicates the position on the finger along the $x$-axis and ranges from o (finger tip) to finger length $L$ (base). The initial overlap $x_{0}$ is limited by the requirement that $f(s)$ must be positive,

$$
\min _{s \geq 0} f(s)=f(0) \geq 0
$$

so

$$
x_{0} \leq \frac{F_{\mathrm{avail}, 0}}{k}-\frac{\varepsilon_{0} h N V_{\max }^{2}}{\left(w+g_{0}\right) k} .
$$

We have named the shape described by (4.14) the 'optim' finger shape.

Figure 4.5 shows the 'optim' shape $f(s)$. A higher value for $\frac{k}{N V_{\max }^{2}}$ results in a more pronounced finger shape. When there are no springs $(k=0)$, the finger shape becomes straight, as is expected because the force of a comb drive with straight fingers is constant. Note that, because both $k$ and $F_{\text {avail, } 0}$ in (4.14) are proportional to $h$, the 'optim' shape is independent of the structure height $h$.

To obtain equally large forces at large displacements with only straight fingers, the gap between straight fingers would have to be equal to the gap between the tip of the straight finger and shaped finger in the 'optim' design. Because this gap is small, the capacitance between the straight fingers would be large too, resulting in a reduced side stability (Grade et al., 2003). For the 'optim' shape, only the gap at the tip of the straight finger is small, while the gap for the rest of the overlap region is larger. This results in an increased side stability when compared with only straight fingers with equal force at large displacements. 


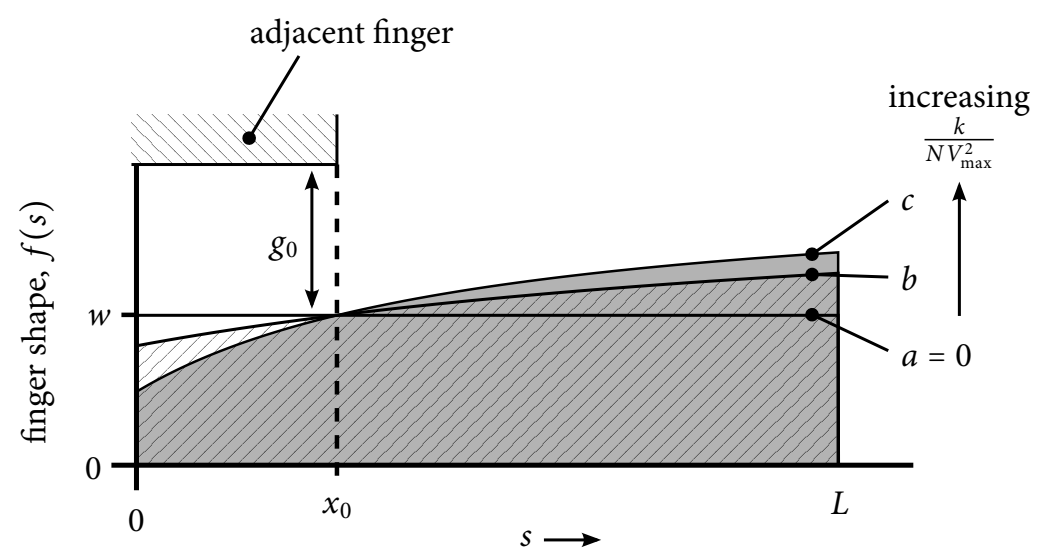

FIGURE 4.5 - Different 'optim' finger shapes $f$, given by (4.14), for different values for spring stiffness $k$, number of finger pairs $N$, and maximum voltage $V_{\text {max }}$. For increasing $k N^{-1} V_{\text {max }}^{-2}$, the finger shape becomes more pronounced $(a=0<b<c)$. The finger width $w$ and gap $g_{0}$ at the initial overlap $x_{0}$ are determined by the fabrication process limits.

\subsubsection{Asymmetric shape}

The obtained result is valid for symmetrically shaped fingers and also for asymmetric fingers with one straight edge and one shaped edge. The unit-cell width of these asymmetric fingers is smaller than for symmetric fingers, and results in more force per unit comb-drive length (see Figure 4.6). In order to obtain the same unit-cell width for symmetrically shaped fingers, the initial overlap $x_{0}$ would have to be reduced.

The sideways forces on the asymmetric finger are unbalanced. The largest sideways force is exerted at the smallest gap size; as can be seen in Figure 4.6(b), the left finger experiences a large downward force at the finger tip, and an upward force somewhere along the finger length depending on the displacement. Especially for large displacements, the difference between the locations of largest force may result in bending of individual fingers. The asymmetric shape also leads to an in-plane torque on the combs; in Figure 4.6(b), the left comb will experience a clockwise torque, the right vice versa. This torque is cancelled by mirroring the finger shape for one half of the comb drive. The middle finger is shaped like the thick finger left in Figure 4.6(a), because it results in the largest force per unit length. Figure 4.1 shows the use of such balanced comb drives with asymmetric fingers.

The found finger shape is similar to the spring softening finger shapes found by Ye et al. (1998) and Jensen et al. (2003), however, these finger shapes result in fingers that are thicker than standard straight fingers, increasing the footprint of the comb drive for the same number of fingers. In our case, the asymmetric 'optim' finger shape leads to $20 \%$ reduction in unit-cell width, compared to the symmetric finger 


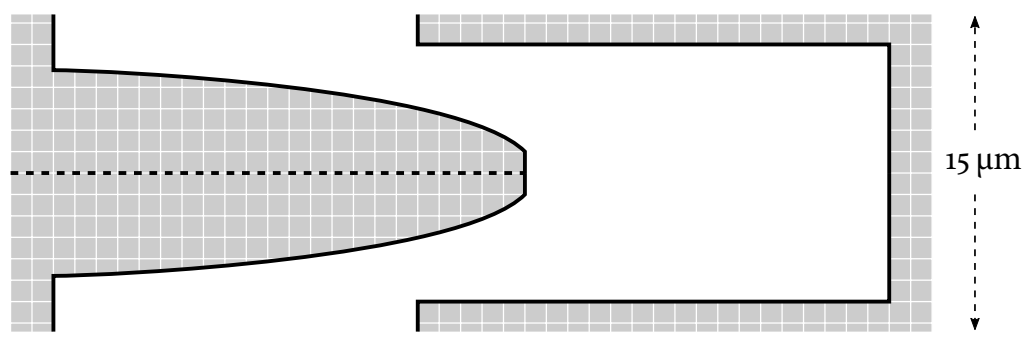

(a) The initial design with symmetric fingers.

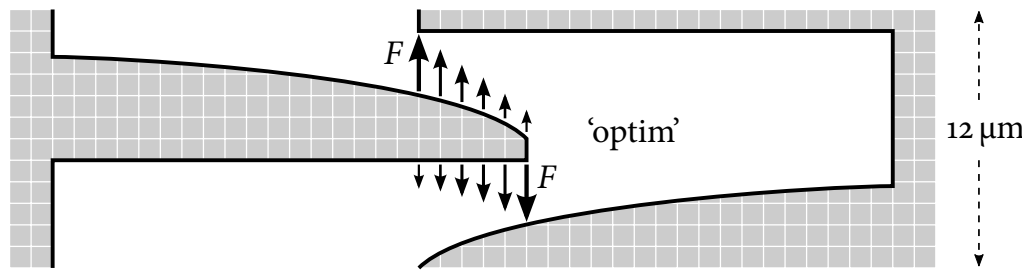

(b) The assymetric space-optimized 'optim' geometry (20\% smaller).

FIGURE 4.6 - Schematic drawings of comb-drive unit cells with optimized fingers. Both geometries have the same force characteristic. The 'optim' shape (b) experiences an asymmetric distributed force; the component perpendicular to $x$ is shown (indicated by $F$ ), where the size of the arrow represents the relative size of the force. Although the total force perpendicular to $x$ cancels on both sides, the asymmetry gives rise to a torque and causes slight bending of the finger (see discussion in text).

shape. The unit-cell width is equal to a comb drive with straight fingers. Therefore, using the finger shape presented in this work will not increase the footprint of the comb drive, and a fair force comparison is made between the unit cells of standard straight and 'optim' shaped fingers.

\subsection{Finite-element analysis}

To validate our analytical model and to obtain information about $F_{\text {comb }}$ when the fingers disengage, the capacitance and $F_{\text {comb }}$ of comb-drive unit cells with straight and 'optim' fingers are calculated using finite-element method (FEM) analysis. A $2 \mathrm{D}$ electrostatic simulation of the unit cells is performed using FreeFEM++ 3.8. Each unit cell contains one finger pair with air in between, see Figure 4.6; the direction of displacement is along the $x$ axis. For each displacement $x$, the electric field $\mathbf{E}$ in the unit cell is calculated, from which the comb drive force is obtained in the following way: The surface charge density $\sigma$ is calculated from the component of the electric field $\mathbf{E}$ normal to the boundary, because along the boundary of a 
conductor

$$
\sigma=\varepsilon \mathbf{E} \cdot \mathbf{n},
$$

where $\varepsilon$ is the permittivity, $\mathbf{n}$ is the unit vector perpendicular to the boundary (direct consequence of Gauss' law, see Griffiths, 1999, p. 102). The comb-drive capacitance is calculated by integrating this surface charge and dividing it by the voltage set in the FEM simulation. The result is shown in Figure 4.7 for both straight and 'optim' fingers. From the simulation result of 'optim' fingers, we obtain that $C(0)=$ 3.o fF for $C(0)$ in (4.12), where we assume the parameter values of Table 4.1. When the fingers are engaged $\left(x \geq-x_{0}=-20 \mu \mathrm{m}\right)$, the result corresponds within $2 \%$ with (4.12). In the disengaged region $\left(x \leq-x_{0}\right)$, the analytical model is not valid and the theoretical value for the capacitance becomes negative. The capacitance goes to zero asymptotically for large negative displacements, as the FEM simulation predicts, and therefore $\frac{\partial C}{\partial x}$ and $F_{\text {comb }}$ will be lower than optimal when the fingers are disengaged. In order to extend the region where the capacitance and force are optimal, the initial finger overlap $x_{0}$ must be increased, because an increase in $C(0)$ moves the intersection of $C(x)$ with the $x$-axis to a lower $x$ value.

The comb-drive force is calculated from the derivative of the FEM calculated capacitance. Figure 4.8 shows the result for the available force. The available force at $x=0, F_{\text {avail, } 0}$, corresponds well with theory, and is the same for both finger shapes, as expected. When the fingers are engaged, the available force for 'optim' fingers is constant, which is as desired. When the fingers disengage, the force drops quickly for both finger shapes; the available (positive) force in the disengaged region is mostly due to the restoring spring suspension force.

The finite-element result confirms that the 'optim' finger shape increases the available force and increases the operation range (grey region in Figure 4.2(a)) compared with straight fingers. The minimum in the available-force curve is higher, and the shock-resistance is therefore larger for 'optim' fingers than straight fingers.

\subsection{Experiment}

\subsubsection{Fabrication}

The comb drives are fabricated from a ( 100$)$ single-crystal highly boron-doped silicon-on-insulator wafer, with a $25 \mu \mathrm{m}$ thick device layer (determining height $h$ of the fingers) and an oxide thickness of $1 \mu \mathrm{m}$. The structures are made by deep reactive-ion etching (DRIE) (Jansen et al., 2009; Laermer and Schilp, 1994), after which the (movable) structures are released by HF vapour phase etching (Holmes and Snell, 1966) of the oxide layer. See Appendix C for a detailed description of the fabrication process.

To fabricate 'optim' fingers, the lithography resolution and the DRIE depth profile are important. While these characteristics are important for all finger shapes, they are especially so for the 'optim' shape, as this shape has a sharp tip and a small finger gap at large displacements. Although we aim to obtain structure widths that 


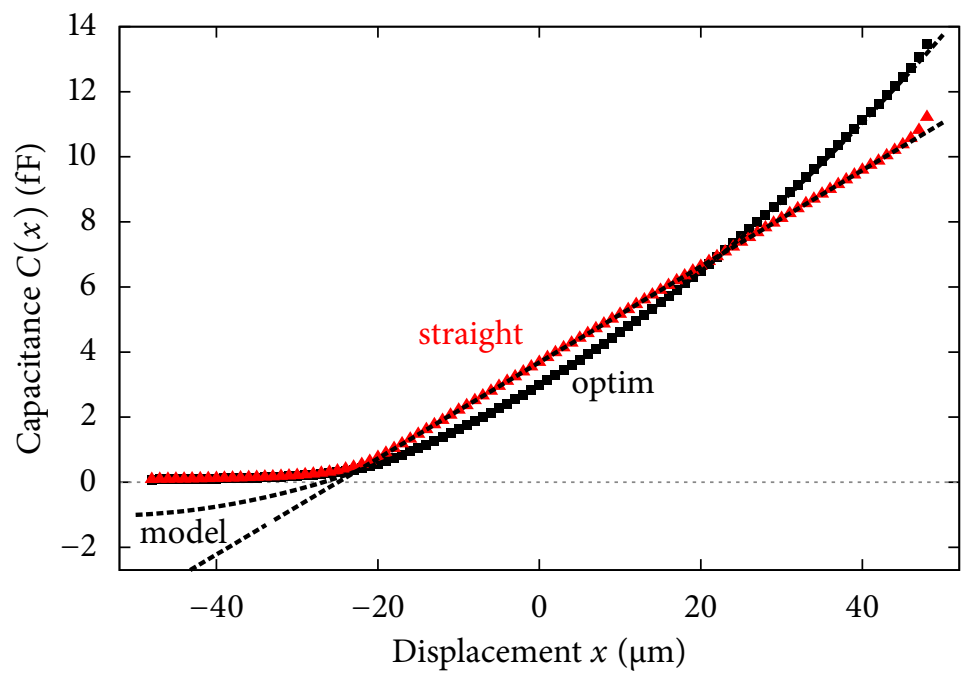

FIGURE 4.7 - Finite-element calculation result: unit-cell capacitance as a function of displacement, for both straight and 'optim' finger shapes. The dashed lines are the designed capacitance of (4.12), where $C(0)=3.7 \mathrm{fF}$ and $C(0)=3.0 \mathrm{fF}$ are used to fit the curves to the FEM results for the straight $(k=0)$ and 'optim' shapes, respectively. The initial overlap $x_{0}$ equals $20 \mu \mathrm{m}$. The fingers are disengaged for $x<-20 \mu \mathrm{m}$, and the analytical model (4.12) is not valid in that region.

are equal to the designed width, small deviations are typical and unavoidable for the used fabrication process. It is better to obtain a thinner than designed finger after fabrication, rather than a thicker than designed finger, because a thicker finger may lead to device failure due to a too small gap at large displacements. We use a positive photoresist, to prevent widening of the fingers due to overexposure.

Equations (4.10) and (4.14) have been applied together with the values in Table 4.1 to define the shape of the comb-drive fingers. Identical folded flexure spring suspensions, with $3 \mu \mathrm{m}$ spring width, are used for each comb drive, therefore $k$ is expected to be the same for each comb drive,

$$
k=2 E h\left(\frac{t}{L_{\text {spring }}}\right)^{3},
$$

where $E$ is the Young's modulus of silicon, and $t$ and $L_{\text {spring }}$ the spring width and length, respectively (Legtenberg et al., 1996). However, because the spring width after fabrication is inherently slightly different from the designed width $t$, the spring stiffness is not precisely known during design. Therefore, several shapes for a range of spring widths and stiffnesses have been fabricated.

Possible issues with the 'optim' shape are voltage breakdown at high displacements, and side snap-in of the comb drive or individual comb fingers. The smallest 


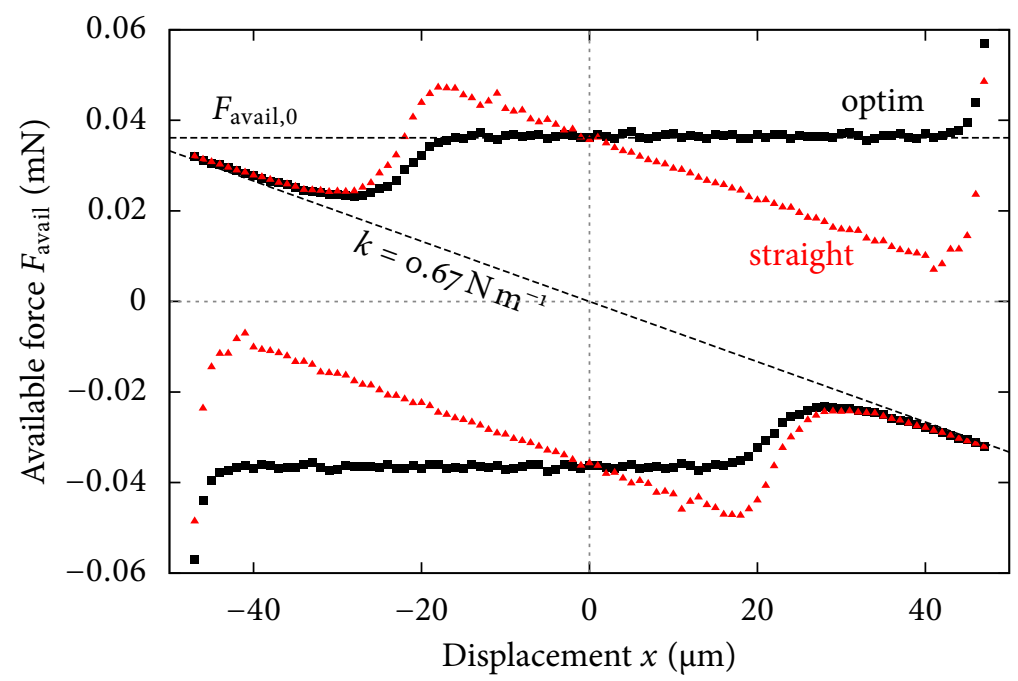

FIGURE 4.8 - Finite-element calculation result: available force as a function of displacement. Table 4.1 lists the values used in the simulation. The theoretical $F_{\text {avail }, 0}$ and the spring force are indicated by dashed lines. The available-force curves are mirrored to obtain the negative available-force curves. The space between the two curves for a finger shape is the range of forces that can be exerted. At $x=-20 \mu \mathrm{m}$, the fingers disengage, causing a steep decrease in force.

TABLE 4.1 - The fabrication process's design constraints, the design parameters, and the resulting expected values for the available force at $x=0$ and spring stiffness.

\begin{tabular}{llll}
\hline Design constraints & Maximum voltage & $V_{\max }$ & $70 \mathrm{~V}$ \\
& Device layer height & $h$ & $25 \mu \mathrm{m}$ \\
& Minimum gap size & $g_{0}$ & $3 \mu \mathrm{m}$ \\
& Finger width & $w$ & $3 \mu \mathrm{m}$ \\
& Spring width & $t$ & $3 \mu \mathrm{m}$ \\
& Young's modulus & $E$ & $169 \mathrm{GN} \mathrm{m}^{-2}$ \\
& Number of finger pairs & $N$ & 100 \\
& Finger length & $L$ & $70 \mu \mathrm{m}$ \\
& Initial overlap & $x_{0}$ & $20 \mu \mathrm{m}$ \\
Expected values & Spring length & $L_{\text {spring }}$ & $700 \mu \mathrm{m}$ \\
& Available force at $x=0$ & $F_{\text {avail, } 0}$ & $36.2 \mu \mathrm{N}$ \\
& Spring stiffness & $k$ & $0.67 \mathrm{~N} \mathrm{~m}^{-1}$ \\
\hline
\end{tabular}




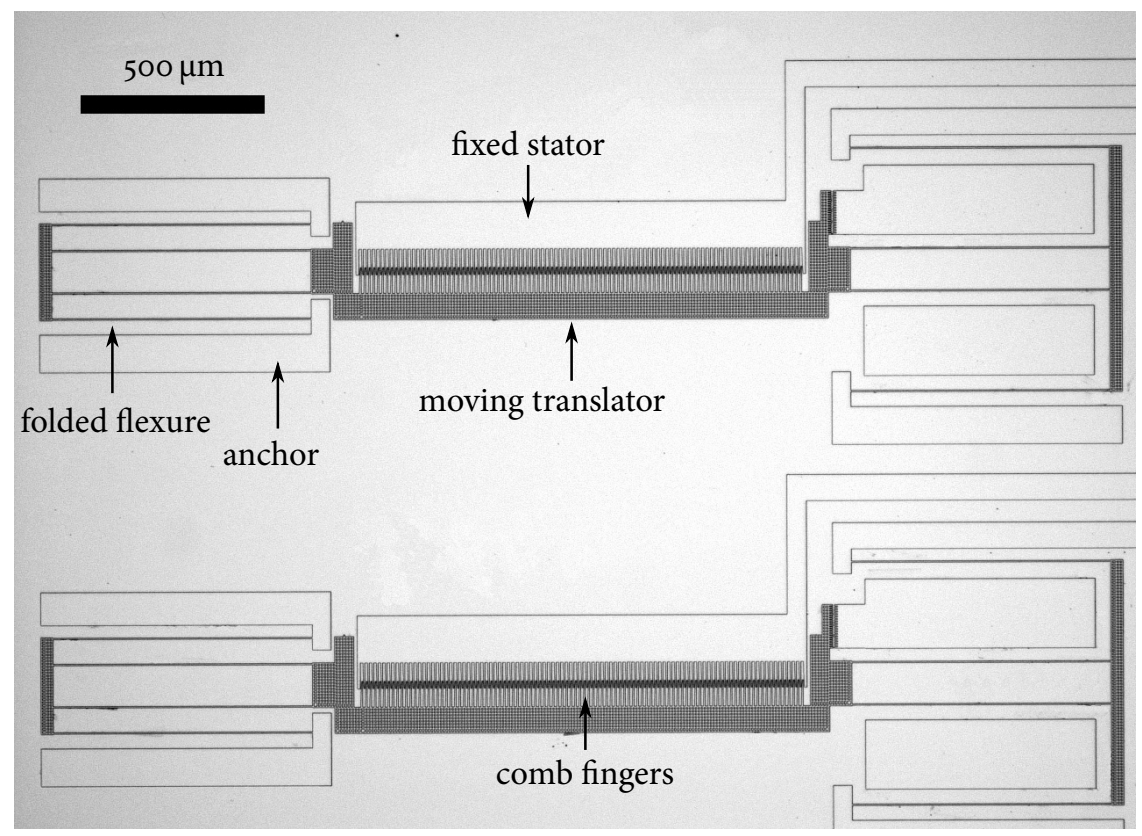

FIGURE 4.9 - Optical micrograph of two comb drives with folded flexure spring suspensions. The moving structures are perforated for silicon oxide underetch, which is why they appear darker in the image.

gap at $35 \mu \mathrm{m}$ displacement is $1.8 \mu \mathrm{m}$, for which a breakdown voltage above $300 \mathrm{~V}$ is expected (Chen et al., 2006), well above the maximum applied voltage of $70 \mathrm{~V}$. The bending of the finger can be estimated by calculating the bending of a cantilever beam due to a force at its end, because the bending force at the tip of the fingers is largest, see Figure 4.6(b). The beam's equivalent thickness is approximated by $3 \mu \mathrm{m}$; and the force is approximated by the force on two parallel plates separated by a gap equal to the $1.8 \mu \mathrm{m}$ tip gap at $35 \mu \mathrm{m}$ displacement, with an area equal to $h l$, where $l$ is the length of the part near the tip with a small gap and large force, which we estimate $l=10 \mu \mathrm{m}$. In this worst case scenario, the bending is $40 \mathrm{~nm}$. Therefore, no observable bending of individual fingers is expected during the measurements.

Figure 4.9 and Figure 4.10 show images of fabricated structures. The 'optim' shape for $t=2.4 \mu \mathrm{m}$ is very subtle, but for $t=3.0 \mu \mathrm{m}$ the asymmetric shape is clearly visible. The tip width for $t=3.0 \mu \mathrm{m}$ is only $1.25 \mu \mathrm{m}$, however, the fabricated tip is only $0.5 \mu \mathrm{m}$ shorter than designed due to rounding. The fabricated shape closely fits the designed shape within $0.2 \mu \mathrm{m}$. 


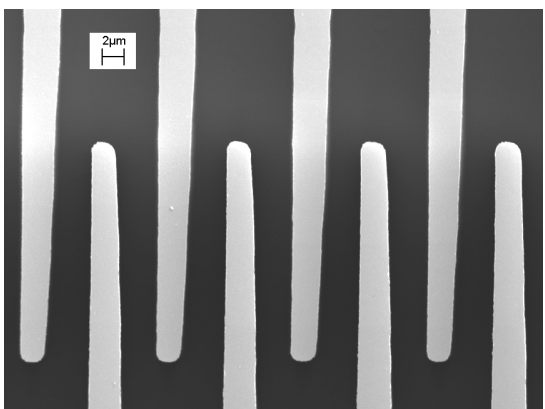

(a) $t=2.4 \mu \mathrm{m}$

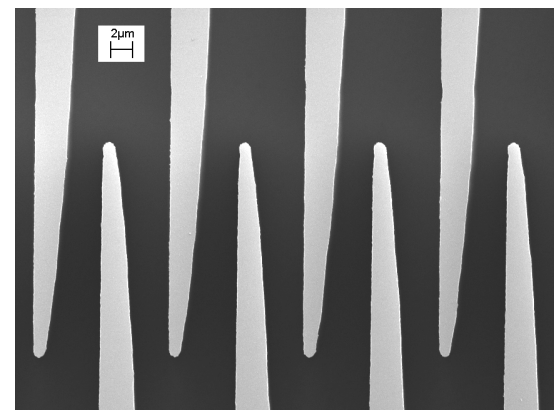

(b) $t=3.0 \mu \mathrm{m}$

FIGURE 4.10 - Scanning electron micrographs of the tips of the fabricated fingers for two different suspension spring stiffnesses (indicated by the spring width $t$ ). The scale bars are $2 \mu \mathrm{m}$ long.

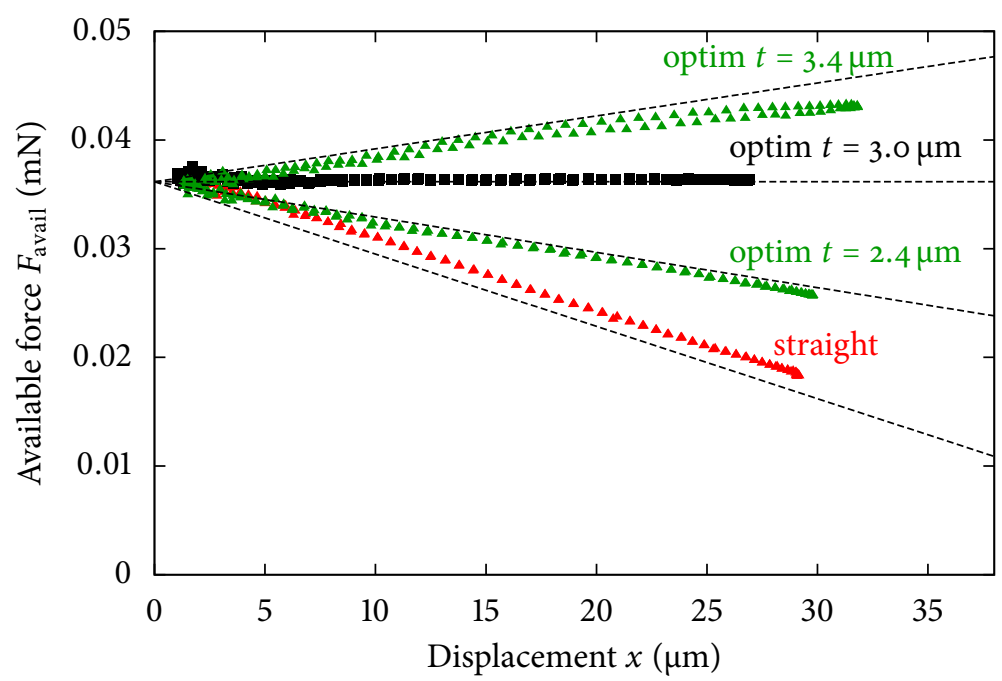

FIGURE 4.11 - The available force as a function of displacement, determined from equilibrium displacement measurements $\left(k=0.67 \mathrm{~N} \mathrm{~m}^{-1}\right)$, of shaped 'optim' fingers designed for spring stiffnesses weaker than $(t=2.4 \mu \mathrm{m})$, equal to $(t=3.0 \mu \mathrm{m})$ and stronger than $(t=3.4 \mu \mathrm{m})$ the actual designed spring stiffness. The measured available force of straight fingers is shown for comparison. The dashed lines are theoretical curves for the straight and the 'optim' finger shapes. 


\subsubsection{Measurement results}

The available-force curve is determined indirectly from equilibrium displacement measurements at equilibrium voltages $V_{\text {eq }}$. These stroboscopic video microscopy measurements are performed with a Polytec MSA40o and its Planar Motion Analyzer software (Planar Motion Analyzer 2.5, Polytec MSA40o, Polytec GmbH). Using the equilibrium condition (4.5),

$$
\frac{\partial C}{\partial x}=\frac{2 k x}{N V_{\mathrm{eq}}^{2}},
$$

which, when combined with (4.3), gives the available force as a function of measurable quantities,

$$
F_{\text {avail }}(x)=k x\left(\frac{V_{\mathrm{max}}^{2}}{V_{\mathrm{eq}}^{2}}-1\right) .
$$

The obtained spring stiffness after fabrication is estimated from resonance frequency $f_{\mathrm{o}}$ measurements, using $k=m\left(2 \pi f_{\mathrm{o}}\right)^{2}$, and corresponds well with the expected value of $0.67 \mathrm{~N} \mathrm{~m}^{-1}$. The mass $m$ was estimated from the area of the design and the silicon thickness, with a combined uncertainty of about $10 \%$.

Figure 4.11 shows the obtained available-force curves of straight fingers and several 'optim' finger shapes designed for different spring stiffnesses. The result shows a constant available force for $t=3.0 \mu \mathrm{m}$ for which the finger shape was matched to the expected suspension spring stiffness. The available force is linearly dependent on the displacement when the finger shape is matched to a weaker $(t=2.4 \mu \mathrm{m})$ or stiffer $(t=3.4 \mu \mathrm{m})$ suspension. Straight fingers show a slightly larger force than expected, however, the slope of the available-force curve (i.e., the dependence on the displacement) is as expected. The available force at a displacement of $25 \mu \mathrm{m}$ is 1.8 times larger for the correctly matched 'optim' fingers than for straight fingers. The obtained displacements were limited around $30 \mu \mathrm{m}$ by side instabilities.

\subsection{Discussion}

The measurements show that the 'optim' shape results in linear available-force curves, see Figure 4.11. Because a change in spring stiffness $\Delta k$ adds a linear curve $\Delta k x$ to the available force, a linear available-force curve indicates that the comb drive is matched to a certain spring stiffness. The $t=3.0 \mu \mathrm{m}$ and $t=3.4 \mu \mathrm{m}$ 'optim' fingers result in a downward and upward available-force curve, respectively, but because the curves are linear, the finger shapes are indeed matched to different spring stiffnesses. We conclude that the 'optim' finger shape given in (4.14) indeed provides a constant available force for a shock-resistant positioning system.

Knowledge of the spring stiffness is required during the design of the finger shape. Most likely in practice this means that one iteration is required to determine the exact suspension spring stiffness. As will be discussed in Chapter 6, there is a variation in spring stiffness across the wafer after fabrication. The effective 
variation in spring width is approximately $\pm 0.2 \mu \mathrm{m}$, resulting in a variation in the obtained available-force curve comparable to the difference between the curves for $t=3.0 \mu \mathrm{m}$ and $t=3.4 \mu \mathrm{m}$ in Figure 4.11. Note that there will also be a variation in obtained finger profiles; a larger spring width is likely to be accompanied by a larger finger width. The increased widths have opposite effects (wider fingers lead to smaller gaps and a larger force), partially cancelling each other.

The FEM results indicate that there is a sharp decrease in $F_{\text {avail }}$ when the fingers disengage. This can be ameliorated by increasing the initial overlap $x_{0}$; however, $x_{0}$ cannot exceed the maximum value given by (4.15). Increasing finger width $w$ increases the maximum value of $x_{0}$; unfortunately, the increased $w$ decreases the force per unit length and the comb-drive width will increase. In practice, the maximum value of $x_{0}$ given by (4.9) cannot be obtained because of the tip rounding that occurs during fabrication. The end of a finger with a very narrow tip cannot be fabricated and the resulting finger is shorter than designed. Consequently, the comb-drive force is reduced, because the finger length $L$ is reduced, which results

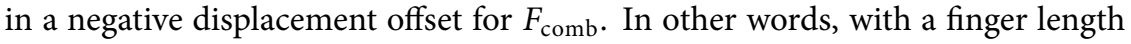
reduced by $\Delta L$ during fabrication, the comb-drive force at $x=0$ is equal to the designed force at $x=-\Delta L$, which is lower than the desired force at $x=0$, see (4.9). In that case, the finger shape (4.14) will not be matched to the designed spring stiffness. This is seen in the result for the $t=3.4 \mu \mathrm{m}$ 'optim' fingers in Figure 4.11, where the actual slope of the available force is lower than the expected slope. With improved fabrication methods allowing very sharp tips, the finger width $w$ could be reduced for more force per unit length, or $x_{0}$ could be increased.

The obtained displacements were limited by side instabilities, indicating that the spring suspension stiffness perpendicular to $x$ is not sufficient at large deflections. To increase the sideways stiffness, improved spring suspension designs can be used, for example suspensions with initially bent beams (Grade et al., 2003) or exact constraint folded flexures (Brouwer et al., 2010).

\subsection{Conclusion}

For shock-resistant actuation, a large available force is desired which is constant over the actuator range. Stability of the available force can be analysed by a graphical method on the force curve. The new 'optim' finger shape presented in this work provides a constant available force, that is as large as possible within the fabrication process's design rules. The analytical solution for this optimal finger shape is given in (4.14). The 'optim' finger shape is asymmetric to reduce the size of the unit cell as is shown in Figure 4.6(b). The unit-cell width of the 'optim' finger shape is equal to a comb drive with straight fingers, such that a fair comparison of the force per unit length is made. Measurements on the fabricated structures show that the presented 'optim' finger shape delivers up to 1.8 times more available force in the measured operation range compared to the straight finger shape. The available force varies linearly with displacement and is constant when the spring constant of the suspension matches the spring stiffness used to calculate the finger shape. 
This constant available force is the optimal solution for shock-resistance, where the minimum available force is as large as possible. When the maximum voltage is applied, the comb-drive acceleration will be large and independent of displacement. The 'optim' finger shape results in comb drives with a large force output and is especially useful in applications where shock-resistant actuation is important, such as portable devices.

\subsection{Acknowledgements}

I would like to thank Kechun 'Kees' Ma, Meint de Boer and Johnny Sanderink for their help in fabricating the structures, and Léon Woldering for helpful discussions. 



\section{Chapter 5}

\section{Energy consumption comparison}

The previous chapters discussed the design of electrostatic comb-drive actuators for use in a parallel probe data storage system. A brief comparison with electrodynamic actuation was already made in Chapter 3. This chapter will expand on this comparison, comparing the seek performance and energy cost of electrostatic comb-drive actuation with other actuation types.

Chapter 2 discusses several actuator types that have been proposed for probe data storage systems, however, in this chapter we will limit the discussion to electrostatic comb-drive (Alfaro and Fedder, 2002; Carley et al., 2001; Kim et al., 2003), electromagnetic comb-drive (Schonhardt et al., 2009), and electrodynamic actuation (Choi et al., 2001a; Huang et al., 2010b; Lantz et al., 2007). To the best of our knowledge, only electrodynamic actuation has been used in a complete probe data storage prototype (by IBM, Pantazi et al., 2008). We believe electrostatic combdrive actuation offers advantages over electrodynamic actuation, especially in terms of energy cost. The electromagnetic comb-drive is interesting, because it mixes aspects of the quite distinct electrostatic comb-drive and electrodynamic actuator. We will assume that the electrostatic and electromagnetic comb-drives use the optim' comb-drive finger shape, optimized for use in a probe data storage system device, described in the previous chapter.

The seek performance of electrostatic comb-drive and electrodynamic actuators has been studied by Griffin et al. (2000), Schlosser (2004), and Khatib et al. (2008). Here, we will expand their results for 'optim' shaped comb-drives. The energy cost of using an electrodynamic actuator in a probe data storage system has been studied by Khatib (2009). However, the difference in energy cost of the different actuator types has not yet been studied in detail. Using the characteristics of the scanner in IBM's prototype (Lantz et al., 2007), hypothetical designs are created for each actuator type, which can then be compared against each other. The performance and energy cost for basic operations (idle, reading/writing, and seeking) is presented. The results can be used in a simulated workload comparison using file system traces (Khatib, 2009) for a more complete comparison.

This chapter has been submitted for publication. 


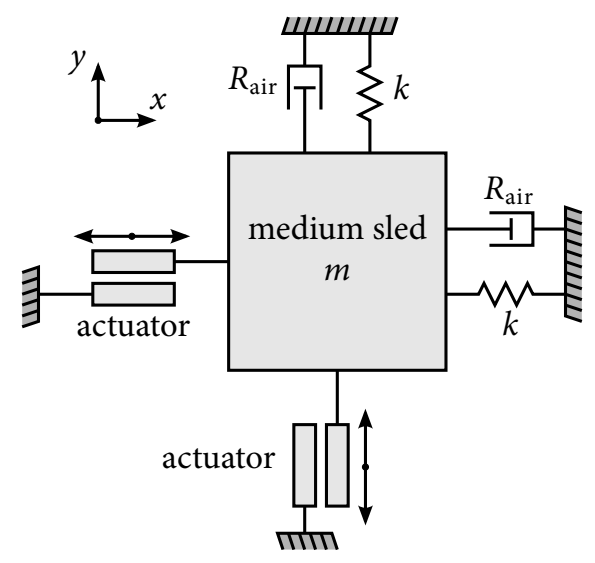

FIGURE 5.1 - General probe storage actuation system.

The damping by air is relatively small and will be neglected in order to simplify the analysis and to obtain more compact equations without losing much accuracy of the estimates. Moreover, the lost energy because of air damping will be similar for all three actuator types. The displacement sensors, control loops and driving circuitry are omitted in the discussion. Here we want to compare only the actuation mechanisms themselves. However, because each actuator type will require a specific type of driving circuitry, future research should try to consider actuator and driving circuitry together for an overall estimate of energy consumption.

\subsection{General aspects}

A general actuation subsystem of a probe data storage device is shown in Figure 5.1. The medium sled, or scan table which carries the storage medium, is suspended by springs such that it is constrained to move along the $x$ and $y$ axes. Two actuators enable independent movement in positive and negative $x$ and $y$ directions. The spring suspension is made such that the $x$ and $y$ axes are almost completely decoupled and can be treated as being independent of each other. Each axis can be modelled accurately by a second-order mass-spring-damper system (Lantz et al., 2007, also see Figure 3.5). The total spring stiffness $k$ is the sum of the stiffnesses of the medium sled suspension and the actuator suspension,

$$
k=k_{\text {table }}+k_{\text {actuator }}
$$

Also, the total actuated mass $m$ is the sum of the masses of the medium sled and the actuator. In case of a mass-balanced system (Chapter 3 ), mass $m$ equals the effective total mass on the actuator side of the pivot. Characteristic values for the actuation subsystem are listed in Table 5.1. Note that the read and write velocities differ. 
TABLE 5.1 - 'Realistic' characteristics of a probe data storage scanner, derived from Lantz et al. (2007) and Cannara et al. (2008).

\begin{tabular}{|c|c|c|}
\hline Quantity & Symbol & Value \\
\hline Actuated mass & $m$ & $100 \mathrm{mg}$ \\
\hline Suspension stiffness & $k$ & $90 \mathrm{~N} \mathrm{~m}^{-1}$ \\
\hline Avail. force at $x=0$ & $F_{\text {avail }, 0}$ & $5 \mathrm{mN}$ \\
\hline Scan range & & $\pm 50 \mu \mathrm{m}$ \\
\hline Read velocity & $v_{\text {read }}$ & $1 \mathrm{~mm} \mathrm{~s}^{-1}$ \\
\hline Write velocity & $v_{\text {write }}$ & $15 \mathrm{~mm} \mathrm{~s}^{-1}$ \\
\hline
\end{tabular}

The displacement $x$ ranges from $-50 \mu \mathrm{m}$ to $50 \mu \mathrm{m}$. The scanner is assumed to be a symmetric device, behaving the same for negative and positive displacements. The actuation mechanisms are symmetric under transformations $x \mapsto-x$ and $y \mapsto-y$. Using this symmetry, the text assumes that all motion is in the positive direction, $x_{0}<x_{1}$. If this is not the case, mirror transformation $\mathcal{M}$ should be applied to obtain correct results,

$$
\mathcal{M}=\{x \mapsto-x, v \mapsto-v\} .
$$

Generally, the $x$ and $y$ axes have different characteristic values, and the scanner is therefore not symmetric under transformation $\{x \mapsto y, y \mapsto x\}$. There is a 'fast' axis with a higher resonance frequency than the other, 'slow', axis. We will assign the $x$ axis to the 'fast' axis along which data is read, and $y$ to the 'slow' axis. Although using a spiral read-out path has advantages for the control system (Kotsopoulos and Antonakopoulos, 2010), we will assume a more standard $x / y$-rasterized read-out path, where the $y$ axis is used to select a row of bits that is read by scanning along the $x$ axis. This is the convention used for example by Eleftheriou et al. (2003), but is opposite to the convention used by Hong and Brandt (2002).

At any moment, the scanner is in one of four modes: idle, stay, read/write, seek. The behaviour of the scanner can be described as a consecutive string of modes. For example: idle, seek(o to 25$), \operatorname{read}(25$ to 26$), \operatorname{seek}(26$ to 12$)$, write(12 to $11)$, stay(11), write(11 to 10$)$, seek $(10,0)^{*}$, idle. In idle mode, no energy is consumed. The energy cost of the other modes will be denoted as follows: (1) staying at a certain displacement $x$ for some time $t, E_{\text {stay }}(x, t)$, (2) moving from $x_{0}$ to $x_{1}$ at speed $v, E_{\text {read }}\left(x_{0}, x_{1}, v\right)$, and (3) moving as quickly as possible from $x_{0}$ with initial velocity $v_{0}$ to a new displacement $x_{1}$ with final velocity $v_{1}, E_{\text {seek }}\left(x_{0}, x_{1}, v_{0}, v_{1}\right)$. The seek time will be denoted as $t_{\text {seek }}\left(x_{0}, x_{1}, v_{0}, v_{1}\right)$. 'Bang-bang' control, where the driving signal is always at its maximum, is assumed for seek operations. 'Bangbang' control is time-optimal for linear systems (LaSalle, 1959). This is also valid for the non-linear scanners discussed here, because the force is, although non-linear, a strictly increasing function of the input voltage or current. The actual control

\footnotetext{
${ }^{*}$ To save energy, one can decide to let the scanner passively settle to zero, instead of actively seeking to the rest position.
} 


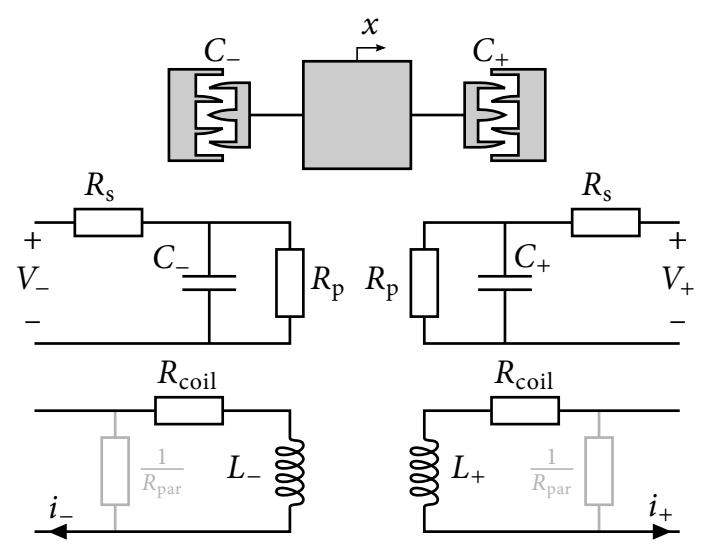

FIGURE 5.2 - Circuits for the $x$ axis of an electrostatic and electromagnetic comb-drive. The circuits are each other's duals, and can be transformed into each other using Table 5.2.

output in IBM's prototype is quite similar to 'bang-bang' control (Sebastian et al., 2006).

\subsection{Comb-drive actuation}

The force generated by an electrostatic comb-drive equals

$$
F_{\mathrm{comb}}=\frac{1}{2} \frac{\partial C}{\partial x} V^{2}
$$

where $x$ is the displacement, $C$ the capacitance between the electrodes, and $V$ the applied voltage (see $\$ 3.1$ ). The generated force is always attractive, meaning that two comb drives are required to actuate in both positive and negative $x$-directions.

Analogous to using electrodes and an electric field to generate a force, it is possible to generate a force using interdigitated magnetic pole shoes and a magnetic field (Schonhardt et al., 2009). The magnetic field is 'applied' by flowing a current through a coil that is magnetically connected to the pole shoes. The generated force $F_{\text {comb,m }}$ equals

$$
F_{\mathrm{comb}, \mathrm{m}}=\frac{1}{2} \frac{\partial L}{\partial x} i^{2}
$$

where $L$ is the total inductance of the magnetic circuit, and $i$ the electrical current through the coil (Schonhardt et al., 2009).

Both the electrostatic and electromagnetic comb-drives suffer from energy losses due to electrical resistances. The electrical circuits of both comb-drive types are shown in Figure 5.2. Because the electrical circuits of the electrostatic and electromagnetic comb-drives are each other's duals (Tilmans, 1996), the same equations 
TABLE 5.2 - Relation between the electrostatic and electromagnetic comb-drive variables.

\begin{tabular}{ccc}
\hline Electrostatic & & Electromagnetic \\
$R_{\mathrm{p}}$ & & $1 / R_{\text {coil }}$ \\
$R_{\mathrm{s}}$ & & $1 / R_{\mathrm{par}}$ \\
$V$ & & $i$ \\
$C$ & $L$ \\
\hline
\end{tabular}

apply for the electrostatic as for the electromagnetic comb-drive, substituting variables as listed in Table 5.2. In the following discussion, we will derive equations for an electrostatic comb-drive, without losing the applicability of the equations to an electromagnetic comb-drive. Note that subscripted symbols should also be substituted, for example $i_{\max }$ for $V_{\max }$.

We assume that the 'optim' finger shape, described in the previous chapter, is used. Note that the 'optim' finger shape, with slight modification, is also applicable to electromagnetic comb-drive actuators. Using 'optim' shaped fingers, the 'positive' comb drive's total capacitance depends non-linearly on the position, see (4.12),

$$
C_{+}(x)=\frac{k x^{2}}{V_{\max }^{2}}+\frac{2 F_{\mathrm{avail}, 0} x}{V_{\max }^{2}}+C(0),
$$

where $C(0)$ represents the capacitance at zero displacement (mostly due to the initial overlap between comb fingers). The 'negative' comb drive's capacitance $C_{-}$ is equal to $C_{+}$with mirrored $x$,

$$
C_{-}(x)=C_{+}(-x)=\frac{k x^{2}}{V_{\max }^{2}}-\frac{2 F_{\mathrm{avail}, 0} x}{V_{\max }^{2}}+C(0) .
$$

The available force of the comb-drive system including the suspension springs is constant (see \$4.1.3),

$$
F_{\text {avail,comb }}(x)=F_{\text {avail, } 0} \text {. }
$$

The voltage $V_{\text {eq }}$ required to position the comb drive in equilibrium at displacement $x$ equals

$$
V_{\mathrm{eq}}(x)= \pm \sqrt{\frac{k|x| V_{\max }^{2}}{k|x|+F_{\mathrm{avai}, 0}}},
$$

adapting (4.13), taking the absolute value of $x$ to allow the equation to be used with negative $x$ values when the 'negative' comb drive is actuated. The absolute value of $x$ is used, to allow for negative displacements when $C_{-}$is charged. Due to the quadratic nature of the force, the sign of the applied voltage is irrelevant. Hereafter, we will assume that the voltages applied to comb drives are positive. 


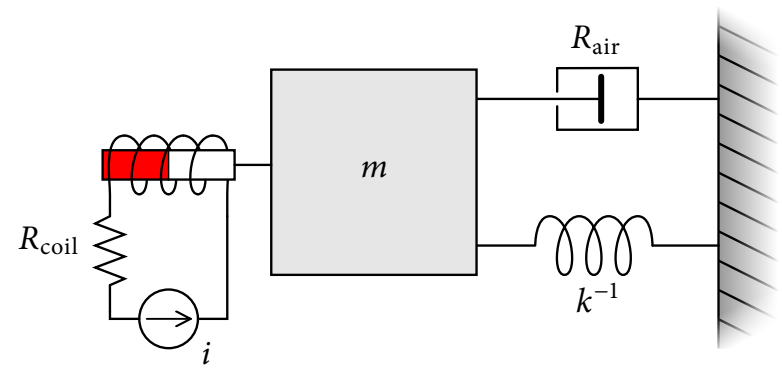

FIGURE 5.3 - Mechanical model of an electrodynamic Lorentz force actuator.

Operating an electrostatic comb-drive involves charging and discharging a capacitance; analogously, operating the electromagnetic actuators involves charging and discharging an inductance. Charging costs energy because energy is stored in the electric field and the spring suspension and because simultaneously energy is dissipated in the series and parallel resistors. The stored energy $E_{\mathrm{c}, \text { stored }}$ in equilibrium equals

$$
E_{c, \text { stored }}(x)=\frac{1}{2} C(x) V_{\mathrm{eq}}^{2}(x)+\frac{1}{2} k x^{2} .
$$

The dissipated energy depends on the charge process. When charging fast, a high current $i$ is required, and much energy is dissipated in $R_{\mathrm{s}}$ because the dissipated energy scales with $i^{2}$. Charging slowly, very little energy is dissipated in $R_{\mathrm{s}}$, however much energy will be dissipated though $R_{\mathrm{p}}$, because there will be a large voltage across $R_{\mathrm{p}}$ for a relatively long time. The optimal charge process for a capacitor, minimizing the energy cost with series and parallel resistors, is calculated in Appendix A. Instead of charging with a constant voltage, it is more energy efficient to charge with an increasing voltage as a function of time. The result shows for our parameter values and charging in $10 \mathrm{~ns}$, that the dissipated energy is two orders of magnitude smaller than the stored energy. We can therefore approximate the required energy to charge the comb-drive capacitance, with resistors, simply by $\frac{1}{2} k x^{2}+\frac{1}{2} C(x) V^{2}$.

\subsection{Electrodynamic actuation}

The probe data storage prototype by IBM uses an electrodynamic actuator with permanent magnets and coils (Vettiger et al., 2002). The actuator uses the Lorentz force generated by flowing a current through a wire in the magnetic field of permanent magnets. Figure 5.3 shows the mechanical model of such an actuator. The permanent magnets and coils can be put in a 'Lorentz-force' configuration (Choi et al., 2001a; Huang et al., 2010b; Rothuizen et al., 2002) or in a voice-coil configuration (Lantz et al., 2007). In the Lorentz-force configuration, the coil slides in or out of the magnetic field, perpendicular to that magnetic field. In the voice-coil configuration, the coil moves between two permanent magnets, parallel to the magnetic 
field. The Lorentz-force and voice-coil configurations have the same characteristics and are treated as one.

The electrodynamic scanners in the literature are (to a good approximation) linear devices (Choi et al., 2001a; Lantz et al., 2007; Rothuizen et al., 2002), where the current required for equilibrium at a certain displacement $x$ is proportional to that displacement,

$$
i_{\mathrm{eq}}(x)=\frac{k x}{n},
$$

where $n$ is the actuator force constant. This linearity implies that the actuator force is proportional to the applied current $i$ and is independent of the displacement,

$$
F_{\mathrm{Ltz}}=n i \text {. }
$$

This is consistent with a flux linkage $\lambda$ that increases linearly with $x$,

$$
\lambda(x, i)=L i+n\left(x+x_{0}\right)
$$

where the inductance $L$ of the coil is constant, and $x_{0}$ is the initial overlap of the coil with the magnetic field. The stored co-energy $E^{\star}(x, i)$ in the actuator (without springs) then equals (Woodson and Melcher, 1968, chap. 3 ),

$$
E^{*}(x, i)=\int_{0}^{i} \lambda\left(x, i^{\prime}\right) \mathrm{d} i^{\prime}=\frac{1}{2} L i^{2}+n\left(x+x_{0}\right) i,
$$

leading to the force when the actuator is current controlled,

$$
F_{\mathrm{Ltz}}=\frac{\partial E^{\star}(x, i)}{\partial x}=n i .
$$

For the Lorentz-force configuration, (5.3) is intuitively correct; (5.3) is also valid for the voice-coil actuator, because there are permanent magnets on both ends of the coil in a push-pull configuration (Lantz et al., 2007). The energy $E$ stored in the actuator equals (Woodson and Melcher, 1968, chap. 3),

$$
E=\lambda i-E^{*}=\frac{1}{2} L i^{2} .
$$

Note that the stored energy is independent of $x$, in contrast to the stored energy for a comb-drive actuator. Whereas for a comb-drive actuator the force stems from the change in energy of both the source and comb drive, the force of the electrodynamic actuator stems from the change in energy of only the source. The total stored energy (including springs) for the electrodynamic actuator equals

$$
E_{\text {stored,Ltz }}=\frac{1}{2} L i^{2}+\frac{1}{2} k x^{2} .
$$

Given a maximum current $i_{\max }$, the maximum actuator force is constant and equals

$$
F_{\max , \mathrm{Ltz}}=i_{\max } n
$$


Therefore, the available force has its maximum at $x=0$ and decreases for increasing displacements, similar to the available force of an electrostatic comb-drive with straight fingers (see Figure 4.2(a)),

$$
F_{\text {avail,Ltz }}(x)=F_{\text {avail,0 }}-k x \text {. }
$$

The maximum obtainable equilibrium displacement $x^{*}$ is the displacement where $F_{\text {avail }}=0$,

$$
x^{*}=\frac{F_{\text {avail }, 0}}{k} .
$$

Like the electromagnetic comb-drive, the dissipative element of the electrodynamic actuator is the resistance $R_{\text {coil }}$ of the coil.

\subsection{Seek time calculations}

To move a linear system from $x_{0}$ to $x_{1}$ as fast as possible, i.e. a time-optimal seek operation, one must use the maximum available force to accelerate and then use the maximum available force to decelerate (LaSalle, 1959). This is called 'bang-bang' control. This applies to the Lorentz actuator where the generated force is independent of position. A comb drive with shaped fingers is a non-linear system, because the generated force depends on the position. However, the comb drive system can be rewritten such that the spring force and comb-drive force are combined as one effective actuator force (effectively becoming a system with $k=0$ ). Using the 'optim' shape, the maximum of this effective actuator force (available force) is constant, and therefore 'bang-bang' control is also time-optimal for 'optim' comb drives.

We define the acceleration $a_{0}$ to be the total maximum acceleration (including springs) at $x=0$ in positive direction,

$$
a_{0}=\frac{F_{\mathrm{avail}, 0}}{m},
$$

defined by the specifications in Table 5.1. The maximum acceleration as a function of position is equal to $a_{0}$ for 'optim' comb drives, in contrast to the electrodynamic actuator for which the maximum acceleration equals $a_{0}-k x$. Because of this difference, the time $t_{\mathrm{hw}}$ and location $x_{\mathrm{hw}}$ of the transition from acceleration to deceleration will be different for each scanner type (the subscript 'hw' stands for 'half-way').

There is an important difference between the fast and slow axes with respect to seeking. This is because for the fast axis, along which data is read/written, there is an initial velocity $v_{0}$ and also a desired final velocity $v_{1}$ after the seek. For the slow axis, both the initial and final velocity are zero.

The calculations below assume that the seek operation can be performed by first applying positive acceleration and subsequent negative acceleration. The correct result for seek operations which first require negative acceleration and subsequent positive acceleration, for example a seek operation for which the start and end 
point are identical, $x_{0}=x_{1}$, with initial velocity zero, $v_{0}=0$, but with a positive final velocity, $v_{1}>0$, is obtained after applying mirror transformation $\mathcal{M}$.

\subsubsection{Comb drives with 'optim' fingers}

For the comb drives, the acceleration during a seek operation is independent of displacement $x$ and is equal to $a_{0}$. The difference between final and initial velocity is therefore equal to $a_{0}$ times the difference between the acceleration and deceleration times,

$$
v_{1}-v_{0}=a_{0}\left(t_{\text {accel }}-t_{\text {decel }}\right) .
$$

The distances travelled during the acceleration and deceleration phases, $x_{\text {accel }}$ and $x_{\text {decel }}$ respectively, are

$$
\begin{aligned}
& x_{\text {accel }}=\int_{0}^{t_{\text {accel }}} v_{0}+a_{0} t \mathrm{~d} t \\
& x_{\text {decel }}=\int_{0}^{t_{\text {decel }}}\left(v_{0}+a_{0} t_{\text {accel }}\right)-a_{0} t \mathrm{~d} t .
\end{aligned}
$$

Combining (5.6), (5.7), and (5.8) with the knowledge that $x_{\text {accel }}+x_{\text {decel }}=x_{1}-x_{0}$, we obtain a solution for the seek time,

$$
t_{\text {seek,comb }}=2 \sqrt{\frac{v_{0}^{2}+v_{1}^{2}}{2 a_{0}^{2}}+\frac{x_{1}-x_{0}}{a_{0}}}-\frac{v_{0}+v_{1}}{a_{0}} .
$$

In order to calculate the seek energy cost, we need to know the half-way point $x_{\mathrm{hw}}$ (equal to $x_{\text {accel }}$ ),

$$
x_{\mathrm{hw}, \mathrm{comb}}=\frac{x_{0}+x_{1}}{2}+\frac{v_{1}^{2}-v_{0}^{2}}{4 a_{0}},
$$

and the half-way time $t_{\mathrm{hw}}$ at which the deceleration phase begins (equal to $t_{\mathrm{accel}}$ ),

$$
t_{\mathrm{hw}, \mathrm{comb}}=\frac{-v_{0}+\sqrt{v_{0}^{2}+2 a_{0}\left(x_{\mathrm{hw}}-x_{0}\right)}}{a_{0}} .
$$

\subsubsection{Electrodynamic actuator}

For the electrodynamic actuator, the available force (5.4) decreases for increasing displacements. This means that seek operations in positive direction will be faster for more negative displacements. Because data is read along the $x$ axis, the seek time along $x$ is more complex to calculate because the start and end velocities are not zero as is the case for the $y$ axis. When the start and/or end velocities are negative, a turn around happens, but the seek operation is still split in one acceleration phase and a subsequent deceleration phase. 
The seek time for an electrodynamic scanner has been calculated by Hong and Brandt (2002). Their calculation of the half-way point assumes that the magnitudes of the start and end velocities are equal, $\left|v_{0}\right|=\left|v_{1}\right|$. The more general solution for the half-way point, valid for all velocities and $x_{0}<x_{1}$ is

$$
x_{\mathrm{hw}, \mathrm{Ltz}}=\frac{k}{4 F_{\mathrm{avail}, 0}}\left(x_{1}^{2}-x_{0}^{2}\right)+\frac{m}{4 F_{\mathrm{avail}, 0}}\left(v_{1}^{2}-v_{0}^{2}\right)+\frac{x_{0}+x_{1}}{2} .
$$

For negative velocities, Hong and Brandt (2002) add an approximate turnaround time to the seek time. However, this turnaround time is only roughly accurate when $\left|v_{0}\right|=\left|v_{1}\right|$. In Appendix B, a more general solution for the seek time is derived, that is valid for all velocities and $x_{0}<x_{1}$,

$$
\begin{aligned}
t_{\text {seek }, \mathrm{Ltz}}= & \sqrt{\frac{m}{k}}\left[\arcsin \left(\frac{x_{\mathrm{hw}}-x^{*}}{\sqrt{\left(x_{0}-x^{*}\right)^{2}+\left(v_{0} \sqrt{\frac{m}{k}}\right)^{2}}}\right)-\arctan \left(\frac{x_{0}-x^{*}}{v_{0} \sqrt{\frac{m}{k}}}\right)+\pi_{0}\right. \\
& \left.+\arcsin \left(\frac{-x_{\mathrm{hw}}-x^{*}}{\sqrt{\left(x_{1}+x^{*}\right)^{2}+\left(v_{1} \sqrt{\frac{m}{k}}\right)^{2}}}\right)+\arctan \left(\frac{x_{1}+x^{*}}{v_{1} \sqrt{\frac{m}{k}}}\right)+\pi_{1}\right],
\end{aligned}
$$

where

$$
\pi_{i}= \begin{cases}\pi & \text { if } v_{i}<0 \\ 0 & \text { if } v_{i} \geq 0\end{cases}
$$

The maximum equilibrium displacement $x^{*}$ is given by (5.5). The half-way time $t_{\mathrm{hw}}$ is equal to $t_{\mathrm{accel}}$ given in Appendix B.

\subsection{Energy cost calculations}

In idle mode, no voltage or current is applied on the actuator and no energy is consumed. The energy cost of the other three modes is discussed below.

\subsubsection{Stay energy}

When the scanner is held in equilibrium away from the origin, the voltage (or current) is held constant and no charging is required. However, still energy must be supplied because of the dissipative elements. For the electrostatic comb-drive, there is leakage through $R_{\mathrm{p}}$; for the electromagnetic comb-drive and the electrodynamic actuator, $R_{\text {coil }}$ is dissipating energy. The dissipated power equals

$$
p_{\text {stay }}(x)=\frac{R_{\mathrm{p}}+R_{\mathrm{s}}}{R_{\mathrm{p}}^{2}} V_{\mathrm{eq}}^{2}(x)=R_{\text {coil }} i_{\mathrm{eq}}^{2}(x) .
$$

The energy cost of standing still at position $x$ for time $t$ equals

$$
E_{\text {stay }}(x, t)=\frac{R_{\mathrm{p}}+R_{\mathrm{s}}}{R_{\mathrm{p}}^{2}} V_{\mathrm{eq}}^{2}(x) \cdot t=R_{\text {coil }} i_{\mathrm{eq}}^{2}(x) \cdot t .
$$




\subsubsection{Read/write energy}

A read/write operation uses a constant velocity $v$ to move from $x_{0}$ to $x_{1}$,

$$
x(t)=x_{0}+v t .
$$

As mentioned in $\$ 5.1$, we assume $x_{0}<x_{1}$; note that this implies that $v>0$.

During read and write operations, the applied voltage (or current) is increased or decreased relatively slowly. Therefore, the charge current during read/write for an electrostatic comb-drive is much lower than the current used when rapidly charging the comb drive. The dissipation of the charge current can therefore be neglected. For the electromagnetic actuators, because $R_{\text {par }}$ is so large, the leakage current can always be neglected. The total energy cost is the sum of the energy cost of an ideal scanner without resistors and the dissipated energy by $R_{\mathrm{p}}$ or $R_{\text {coil }}$,

$$
E_{\text {read }}\left(x_{0}, x_{1}, v\right)=E_{\text {read,ideal }}\left(x_{0}, x_{1}\right)+E_{\text {read,diss }}\left(x_{0}, x_{1}, v\right) \text {. }
$$

The energy cost of a read/write operation for an ideal scanner is equal to the change in stored energy. Because the velocity is constant, there is no change in kinetic energy.

When both $x_{0}$ and $x_{1}$ are negative, $x_{0}<x_{1}<0$, the (negative) actuator and spring should be slowly discharged, and no extra energy has to be supplied.

When $x_{0}$ and $x_{1}$ have opposite signs, $x_{0}<0<x_{1}$, the stored energy at $x_{0}$ cannot easily be used to load the spring in the positive direction or to charge the actuator up to $x_{1}$, because the directions are opposite. The stored energy at $x_{0}$ is completely discarded, before charging the system to $x_{1}$.

When $x_{0}$ and $x_{1}$ are both positive, the extra required energy is the difference between the stored energy at $x_{0}$ and $x_{1}$.

These three cases can be summarized as follows,

$$
E_{\text {read,ideal }}\left(x_{0}, x_{1}\right)= \begin{cases}0 & \text { if } x_{0}<x_{1} \leq 0, \\ E_{\text {stored }}\left(x_{1}\right) & \text { if } x_{0} \leq 0<x_{1}, \\ E_{\text {stored }}\left(x_{1}\right)-E_{\text {stored }}\left(x_{0}\right) & \text { if } 0<x_{0}<x_{1} .\end{cases}
$$

The dissipated energy is the integral of the dissipated power $p_{\text {diss }}$,

$$
E_{\text {read,diss }}\left(x_{0}, x_{1}, v\right)=\int_{0}^{\frac{x_{1}-x_{0}}{v}} p_{\text {diss }} \mathrm{d} t .
$$

The dissipated power is equal to $p_{\text {stay }}$, see (5.10). Using (5.11),

$$
E_{\text {read,diss }}\left(x_{0}, x_{1}, v\right)=\frac{1}{v} \int_{x_{0}}^{x_{1}} p_{\text {stay }}(x) \mathrm{d} x,
$$

Because the equilibrium voltage and current are the same for the electrostatic and electromagnetic comb-drives, the integral can be worked out for both comb drives simultaneously. However, the details are different for the Lorentz actuator. 


\section{Comb drives with 'optim' fingers}

Working out the integral in (5.12) for 'optim' comb drives, using (5.3) and (5.10), we find

$$
E_{\text {read,diss,comb }}\left(x_{0}, x_{1}, v\right)=\frac{R_{\mathrm{p}}+R_{\mathrm{s}}}{R_{\mathrm{p}}^{2}} \frac{V_{\max }^{2}}{v} \int_{x_{0}}^{x_{1}} \frac{|x|}{|x|+\frac{F_{\text {avail }, 0}}{k}} \mathrm{~d} x \text {. }
$$

Because $p_{\text {diss }}$ only depends on the instantaneous position and not on the direction of motion, the dissipated energy moving from $x_{0}$ to $x_{1}$ is equal to the dissipated energy when moving from $x_{1}$ to $x_{0}$. Moreover, because the scanner is symmetric, the dissipated energy is equal for moving from 0 to $x$ and moving from $-x$ to 0 . Defining an intermediate solution $f$ equal to the total dissipated energy moving from 0 to $x$, or from $x$ to 0 when $x<0$,

$$
f(x)=\frac{R_{\mathrm{p}}+R_{\mathrm{s}}}{R_{\mathrm{p}}^{2}} \frac{V_{\max }^{2}}{v} \int_{0}^{|x|} \frac{x^{\prime}}{x^{\prime}+\frac{F_{\text {avail }, 0}}{k}} \mathrm{~d} x^{\prime},
$$

we obtain

$$
\begin{aligned}
E_{\text {read,diss,comb }}\left(x_{0}, x_{1}, v\right) & =\operatorname{sgn}\left(x_{1}\right) f\left(x_{1}\right)-\operatorname{sgn}\left(x_{0}\right) f\left(x_{0}\right), \\
f(x) & =\frac{R_{\mathrm{p}}+R_{\mathrm{s}}}{R_{\mathrm{p}}^{2}} \frac{V_{\text {max }}^{2}}{v}\left[|x|-\frac{F_{\text {avail }, 0}}{k} \ln \left(\frac{k|x|+F_{\text {avail }, 0}}{F_{\text {avail }, 0}}\right)\right],
\end{aligned}
$$

where $\operatorname{sgn}(x)$ is the sign function.

Note that decreasing $V_{\max }$ decreases the dissipated energy. However, the combdrive size is inversely proportional to $V_{\max }^{2}$ (to obtain an equal $F_{\text {avail,o }}$ ), and $R_{\mathrm{p}}$ may decrease proportionally with the comb-drive size because the area where leakage occurs increases, depending on the design. Therefore, the effect of decreasing $V_{\max }$ may be cancelled by a decrease in $R_{\mathrm{p}}$.

\section{Electrodynamic actuator}

Working out the integral in (5.12) for the electrodynamic actuator, we find

$$
E_{\text {read,diss,Ltz }}\left(x_{0}, x_{1}, v\right)=R_{\text {coil }} \frac{k^{2}}{3 v n^{2}} \cdot\left(x_{1}^{3}-x_{0}^{3}\right) \text {. }
$$

\subsubsection{Seek energy}

During a seek operation, the actuator moves from $x_{0}$ to $x_{1}$ as fast as possible, with initial velocity $v_{0}$ and final velocity $v_{1}$. The calculations below assume that the seek operation can be performed by first accelerating the sled and subsequently decelerating it, see $\$ 5.4$. 


\section{Comb drives with 'optim' fingers}

Seeking from $x_{0}$ to $x_{1}$ using comb drives can be subdivided in five steps: (1) $C_{-}$is discharged and $C_{+}$is charged to $V_{\max }$, (2) the sled is accelerated while the voltages are kept constant, (3) at $x_{\mathrm{hw}}, C_{+}$is discharged and $C_{-}$is charged to $V_{\max }$, (4) the sled is decelerated while the voltages are kept constant, and finally (5) at $x_{1}, C_{+}$or $C_{-}$is charged up to $V_{\mathrm{eq}}\left(x_{1}\right)$, for positive or negative $x_{1}$, respectively. Because the mechanical resonance is much slower than the electrical resonance $\left(\sqrt{m k^{-1}} \gg R C\right)$, the charging and discharging of $C_{-}$and $C_{+}$is much faster than the movement of the sled. Therefore, the capacitance is considered constant during steps 1 and 3 .

The energy cost of a seek operation is the sum of five terms corresponding to the five steps:

$E_{1}$ Charge $C_{+}$.

$$
E_{1}=\frac{1}{2} V_{\max }^{2} C_{+}\left(x_{0}\right) .
$$

$E_{2}$ Keep voltage constant on $C_{+}$while it is moving. The required supply current $i$ is equal to the current required to maintain $V_{\max }$ across the comb drive plus the leakage current through $R_{\mathrm{p}}$,

$$
i=V_{\max } \frac{\mathrm{d} C_{+}}{\mathrm{d} t}+\frac{V_{\max }}{R_{\mathrm{p}}} .
$$

Using the values from Table 5.1 and Table 5.3, the maximum current is on the order of tens of microamperes; the maximum voltage drop over $R_{\mathrm{s}}$ is on the order of millivolts and therefore negligible (note that this is also valid for the electromagnetic comb drive, because, in that case, $R_{\mathrm{s}} \approx 0$ ). Because charging is relatively slow, the current is so small that dissipation in $R_{\mathrm{s}}$ can be neglected. The required energy equals

$$
E_{2}=\int_{0}^{t_{\mathrm{hw}}} V_{\max } i \mathrm{~d} t=V_{\max }^{2}\left[C_{+}\left(x_{\mathrm{hw}}\right)-C_{+}\left(x_{0}\right)+\frac{t_{\mathrm{hw}}}{R_{\mathrm{p}}}\right] .
$$

This energy $E_{2}$ minus the dissipative term is equal to the sum of the extra stored energies of the electric field in the comb drive, the springs and the kinetic energy of the moving mass, as can easily be confirmed using (5.2).

$E_{3}$ Discard energy in $C_{+}$, charge $C_{-}$.

$$
E_{3}=\frac{1}{2} V_{\max }^{2} C_{-}\left(x_{\mathrm{hw}}\right) \text {. }
$$

$E_{4}$ Keep voltage constant on $C_{-}$while it is moving.

$$
E_{4}=\max \left(V_{\max }^{2}\left[C_{-}\left(x_{1}\right)-C_{-}\left(x_{\mathrm{hw}}\right)+\frac{t_{\mathrm{seek}}-t_{\mathrm{hw}}}{R_{\mathrm{p}}}\right], 0\right) .
$$

Note that because the difference $C_{-}\left(x_{1}\right)-C_{-}\left(x_{\mathrm{hw}}\right)$ is negative, the total required energy including dissipation through $R_{\mathrm{p}}$ can be negative. Therefore, $E_{4}$ has an explicit lower limit of zero, because it is assumed that the excess energy is not regained. 
$E_{5}$ Charge $C_{+}$or $C_{-}$to the equilibrium voltage $V_{\text {eq }}\left(x_{1}\right)$. If $x_{1}<0, C_{-}$should be charged to $V_{\mathrm{eq}}\left(x_{1}\right)$, but because $C_{-}$is already charged at $V_{\max }, C_{-}$has to be discharged which does not consume energy. For positive $x_{1}, C_{+}$must be charged from zero.

$$
E_{5}= \begin{cases}0 & \text { if } x_{1} \leq 0, \\ \frac{1}{2} V_{\mathrm{eq}}^{2}\left(x_{1}\right) C_{+}\left(x_{1}\right) & \text { if } x_{1}>0 .\end{cases}
$$

The total seek energy equals

$$
E_{\text {seek }}\left(x_{0}, x_{1}, v_{0}, v_{1}\right)=E_{1}+E_{2}+E_{3}+E_{4}+E_{5} .
$$

\section{Electrodynamic actuator}

Seeking from $x_{0}$ to $x_{1}$ using an electrodynamic scanner can be subdivided in five steps similar to the steps for a comb-drive scanner. The charging steps are slightly different, because an electrodynamic scanner has one transducer (the coil) and a comb-drive scanner has two transducers (one comb drive for positive, and another for negative forces). First, (1) the coil is charged to $+i_{\max }$, then (2) the sled is accelerated while the current is kept constant, (3) at $x_{\mathrm{hw}}$, the coil is discharged and subsequently charged to $-i_{\max }$, (4) the sled is decelerated while the current is kept constant, and finally (5) at $x_{1}$, the coil is charged to $i_{\text {eq }}\left(x_{1}\right)$. Because the mechanical resonance is much slower than the electrical resonance $\left(\sqrt{m k^{-1}} \gg \frac{L}{R}\right)$, the charging and discharging of the coil is much faster than the movement of the sled. Therefore, the displacement $x$ is considered constant during steps 1 and 3 .

The energy cost of a seek operation is the sum of five terms corresponding to the five steps:

$E_{1}$ Charge $L$.

$$
E_{1}=\frac{1}{2} L i_{\max }^{2} .
$$

$E_{2}$ Keep current constant through the coil while it is moving. The required supply voltage $V$ is equal to the electromotive voltage generated by the coil plus the voltage across the series resistance $R_{\text {coil }}$,

$$
V=\frac{\mathrm{d} \lambda}{\mathrm{d} t}+i_{\max } R_{\text {coil }} \text {. }
$$

The required energy equals

$$
E_{2}=\int_{0}^{t_{\mathrm{hw}}} i_{\max } V \mathrm{~d} t=i_{\max } n\left(x_{\mathrm{hw}}-x_{0}\right)+i_{\max }^{2} R_{\mathrm{coil}} t_{\mathrm{hw}} .
$$

This energy $E_{2}$ minus the dissipative term is equal to the sum of the extra stored energies of the springs and the kinetic energy of the moving mass, as can easily be confirmed from integration of the net force on the mass,

$$
\Delta E_{\mathrm{kin}}=\int_{x_{0}}^{x_{\mathrm{hw}}} i_{\max } n-k x \mathrm{~d} x=i_{\max } n\left(x_{\mathrm{hw}}-x_{0}\right)-\frac{1}{2} k\left(x_{\mathrm{hw}}^{2}-x_{0}^{2}\right) .
$$


$E_{3}$ Charge coil with opposite current $-i_{\max }$.

$$
E_{3}=\frac{1}{2} L i_{\max }^{2}
$$

$E_{4}$ Keep current constant through the coil while it is moving. The energy generated by the coil may be more than the dissipated energy in $R_{\text {coil }}$. Therefore, $E_{4}$ has an explicit lower limit of zero, because it is assumed that the excess energy cannot be regained.

$$
E_{4}=\max \left(-i_{\max } n\left(x_{1}-x_{\mathrm{hw}}\right)+i_{\max }^{2} R_{\mathrm{coil}}\left(t_{\mathrm{seek}}-t_{\mathrm{hw}}\right), 0\right) .
$$

$E_{5}$ Charge the coil to the equilibrium current $i_{\mathrm{eq}}\left(x_{1}\right)$. If $x_{1}<0$, the coil is already charged at $-i_{\max }$ and only needs discharging to reach $i_{\mathrm{eq}}\left(x_{1}\right)$, which does not consume energy. For positive $x_{1}$, the coil must be charged from zero current.

$$
E_{5}= \begin{cases}0 & \text { if } x_{1} \leq 0 \\ \frac{1}{2} L i_{\mathrm{eq}}^{2}\left(x_{1}\right) & \text { if } x_{1}>0\end{cases}
$$

The total seek energy equals

$$
E_{\text {seek }}\left(x_{0}, x_{1}, v_{0}, v_{1}\right)=E_{1}+E_{2}+E_{3}+E_{4}+E_{5} .
$$

\subsection{Results for hypothetical designs}

In order to compare the actuator types quantitatively, designs, which are not actually implemented, are made that meet the requirements specified in Table 5.1. The specification of the available force at $x=0$ is used together with the maximum current or maximum voltage to determine the inductance or capacitance of the actuator. The characteristics of the hypothetical devices are listed in Table 5.3.

The initial capacitance $C(0)$ of the electrostatic comb-drive includes a typical bond pad capacitance on the order of $1 \mathrm{pF}$ (Ker et al., 2001). The initial inductance $L(0)$ of the electromagnetic comb-drive is a best-case estimate for which $L_{+}(x)$ in (5.2) equals zero at $-50 \mu \mathrm{m}$ such that $L_{+}(x)$ and $L_{-}$are positive over the whole scan range. This estimate is too optimistic, because the inductance of the actuator at maximum negative displacement is a significant proportion of the total inductance (Schonhardt et al., 2009). The inductance $L$ of the electrodynamic actuator is estimated from the coil dimensions in the design by Lantz et al. (2007).

The resistance values are estimates based on measurements on fabricated devices, private communication with Mark Lantz, IBM Zürich, and the article by Schonhardt et al. (2009). As a first estimate, equal values for the maximum current and coil resistance are taken for the electromagnetic comb-drive and the electrodynamic actuator. 
TABLE 5.3 - Actuator design specifications that meet the requirements of Table 5.1 (based on private communication with Mark A. Lantz, IBM Zürich, and Schonhardt et al., 2009).

\begin{tabular}{llll}
\hline Electrostatic & Initial capacitance & $C(0)$ & $15 \mathrm{pF}$ \\
comb-drive & Maximum voltage & $V_{\max }$ & $150 \mathrm{~V}$ \\
& Series resistance & $R_{\mathrm{s}}$ & $100 \Omega$ \\
& Parallel resistance & $R_{\mathrm{p}}$ & $100 \mathrm{M} \Omega$ \\
Electromagnetic & Initial inductance & $L(0)$ & $2.75 \mathrm{mH}$ \\
comb-drive & Maximum current & $i_{\max }$ & $10 \mathrm{~mA}$ \\
& Series resistance & $R_{\text {coil }}$ & $30 \Omega$ \\
& Parallel resistance & $R_{\mathrm{par}}$ & $\infty$ \\
Electrodynamic & Inductance & $L$ & $25 \mu \mathrm{H}$ \\
& Maximum current & $i_{\max }$ & $10 \mathrm{~mA}$ \\
& Force constant & $n$ & $0.5 \mathrm{NA}^{-1}$ \\
& Series resistance & $R_{\text {coil }}$ & $30 \Omega$ \\
& Parallel resistance & $R_{\mathrm{par}}$ & $\infty$ \\
\hline
\end{tabular}

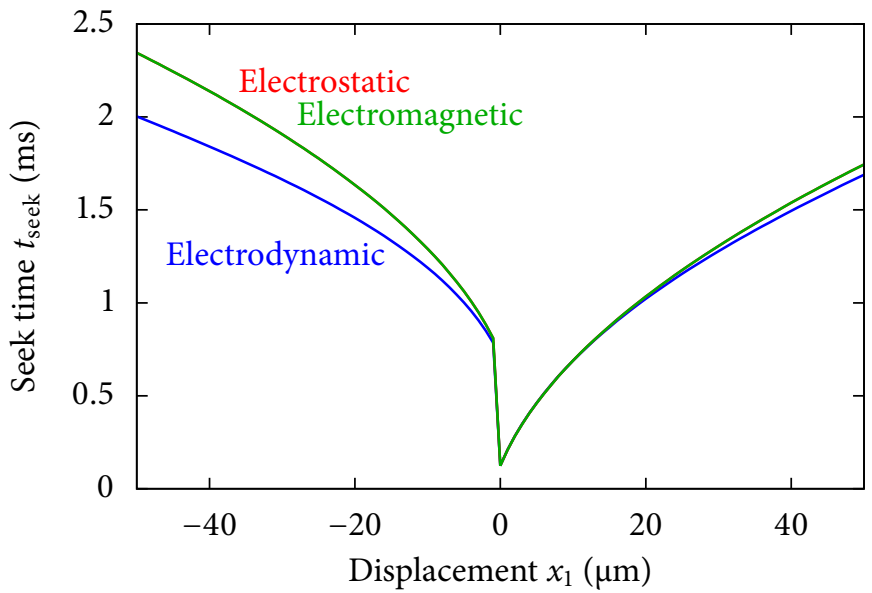

FIGURE 5.4 - The seek time for seeking from $x_{0}=0$ to $x_{1}$, with an initial velocity $v_{0}=0$ and a final velocity $v_{1}=v_{\text {write }}=15 \mathrm{~mm} \mathrm{~s}^{-1}$. The curves for the electrostatic and electromagnetic comb drives overlap. 


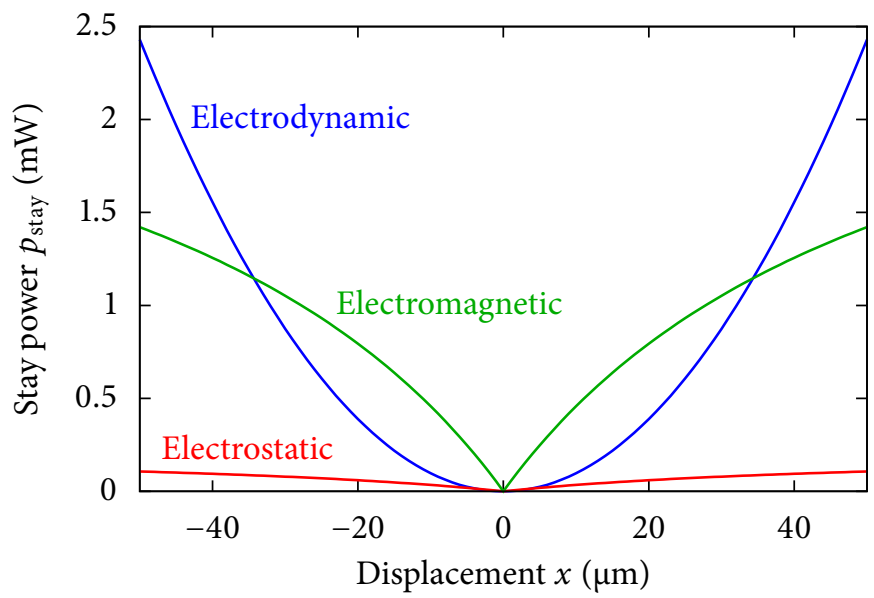

FIGURE 5.5 - The dissipated power versus displacement in equilibrium, $p_{\text {stay }}$.

\subsubsection{Seek time}

The time for a seek from $x_{0}=0$ to $x_{1}$, with an initial velocity $v_{0}=0$ and a final velocity $v_{1}=v_{\text {write }}$ is plotted in Figure 5.4, for the three actuator types. Because the available force characteristics of the comb drives are equal, the seek performance is equal too. Although the electrodynamic available force is quite different from the comb-drive available force, the electrodynamic seek performance is roughly the same but slightly faster. The higher deceleration force compensates for the lower acceleration force compared to the comb drives.

A seek time well within $2 \mathrm{~ms}$ is obtained for most seeks. Note that, in this case starting at rest from zero, negative seeks take $0.8 \mathrm{~ms}$ longer, because of the desired positive writing velocity at the end of the seek, requiring a turn around.

\subsubsection{Stay energy}

Figure 5.5 shows the dissipated power for equilibrium displacements. Although the precise equilibrium currents are different for the electrodynamic actuator and the electromagnetic comb-drive, the average dissipated power figures are on the same order of magnitude. The electrodynamic actuator is more energy efficient around zero, but the electromagnetic comb-drive is more energy efficient at large displacements. The electrostatic comb-drive dissipation is 13 times smaller than the electromagnetic comb-drive for our set of parameters, because

$$
i_{\text {eq }}^{2} R_{\text {coil }} \approx 13 \frac{V_{\mathrm{eq}}^{2}}{R_{\mathrm{p}}} .
$$




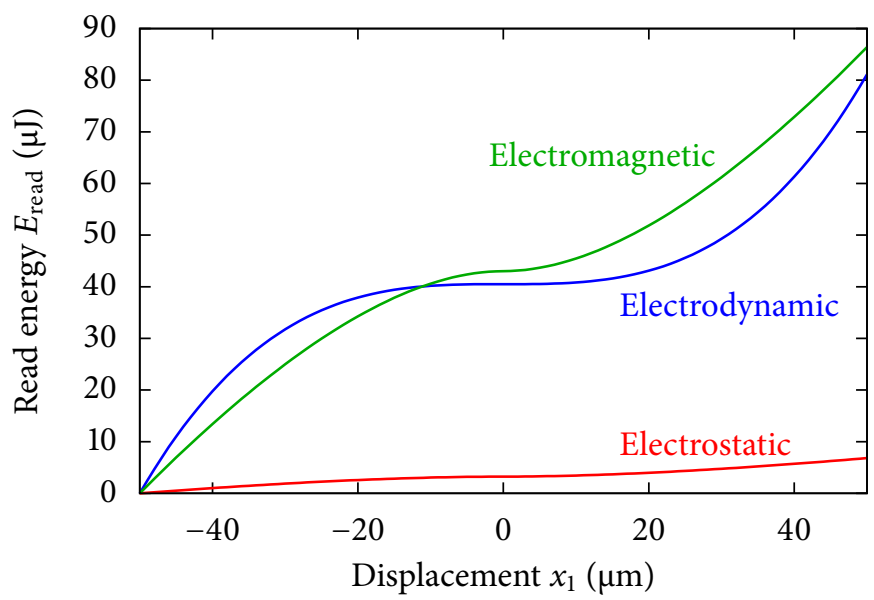

FIGURE 5.6 - The energy cost of moving the scan table at read velocity, $v_{\text {read }}$, starting from $x_{0}=-50 \mu \mathrm{m}$, versus the end displacement $x_{1}$. The energy cost of reads from different $x_{0}$ can be obtained by subtracting the value at $x_{0}$ from the value at $x_{1}$.

The electrodynamic actuator requires about the same power as the electrostatic comb-drive when $|x|<5 \mu \mathrm{m}$, but it consumes an order of magnitude more energy when $|x|>30 \mu \mathrm{m}$.

\subsubsection{Read/write energy}

Figure 5.6 shows the energy cost of read operations starting at $x_{0}=-50 \mu \mathrm{m}$ with velocity $v_{\text {read }}$, as a function of end displacement $x_{1}$. Because

$$
E_{\text {read }}\left(x_{b}, x_{c}, v\right)=E_{\text {read }}\left(x_{a}, x_{c}, v\right)-E_{\text {read }}\left(x_{a}, x_{b}, v\right),
$$

the energy cost for a general read operation from $x_{0}$ to $x_{1}$ at velocity $v$ can be obtained from Figure 5.6 by subtracting the value at $x_{0}$ from the value at $x_{1}$. $E_{\text {read }}$ scales approximately inversely proportional with the velocity,

$$
E_{\text {read }}\left(x_{0}, x_{1}, v_{a}\right) \approx \frac{v_{b}}{v_{a}} E_{\text {read }}\left(x_{0}, x_{1}, v_{b}\right)
$$

therefore, write operations ( $\left.v=v_{\text {write }}\right)$ consume approximately 15 times less energy.

The electromagnetic comb-drive and the electrodynamic actuator consume roughly equal amounts of energy for read/write operations (maximally $20 \%$ difference). The electrostatic comb-drive consumes one order of magnitude less energy than the other actuator types during read/write operations. 


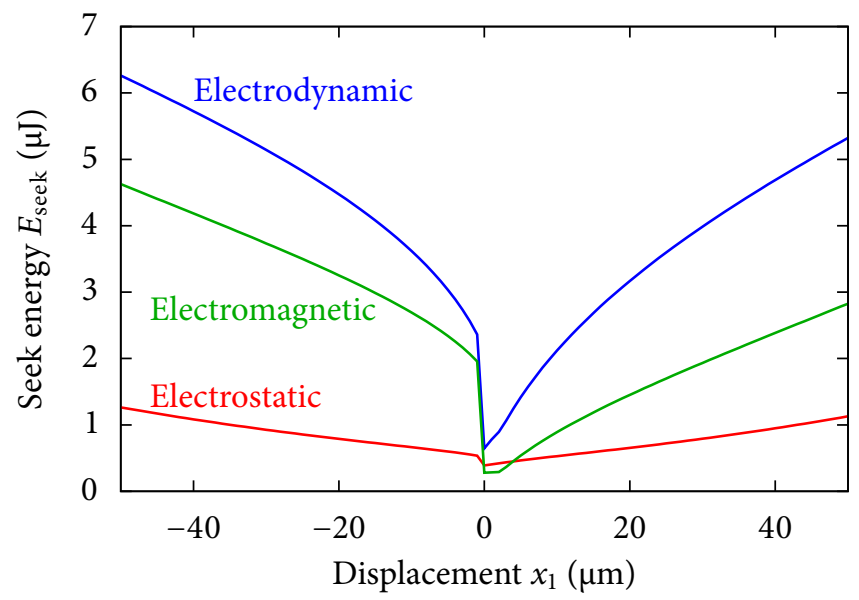

FIGURE 5.7 - The energy cost of seeking from $x_{0}=0$ to $x_{1}$, with an initial velocity $v_{0}=0$ and a final velocity $v_{1}=v_{\text {write }}$. Seeking in the negative direction costs more energy, because of the desired positive final velocity requiring a turn around.

\subsubsection{Seek energy}

The energy cost of a seek operation starting at rest and ending at write speed at displacement $x_{1}$ is shown in Figure 5.7 for the three investigated actuator types. Note that seeking in the negative direction costs more energy, because of the desired positive final velocity, requiring a turn around. The electrostatic comb-drive consumes the least amount of energy.

Because many different kinds of seeks are possible, with different end and start locations and different start and end velocities, a 'Monte Carlo' analysis is done, randomly choosing values for $x_{0}, x_{1}, v_{0}$, and $v_{1}$. The result is shown in Figure 5.8, by plotting for each seek $i$ the energy cost for the electrodynamic actuator and the electromagnetic comb-drive relative to the energy cost for the electrostatic comb-drive. On average, the electrodynamic actuator and the electromagnetic comb-drive use 5.8 and 3.4 times more energy, respectively, than the electrostatic comb-drive. For very short seeks, the energy cost is almost equal for all three actuators.

\subsection{Discussion}

The seek performance of the electrostatic and electromagnetic comb-drives, and the electrodynamic actuator are approximately equal, because their design is such that their force at $x=0$ is equal. Because the performance is similar, the energy cost comparison provides a measure about the relative energy efficacy of each actuator type. 


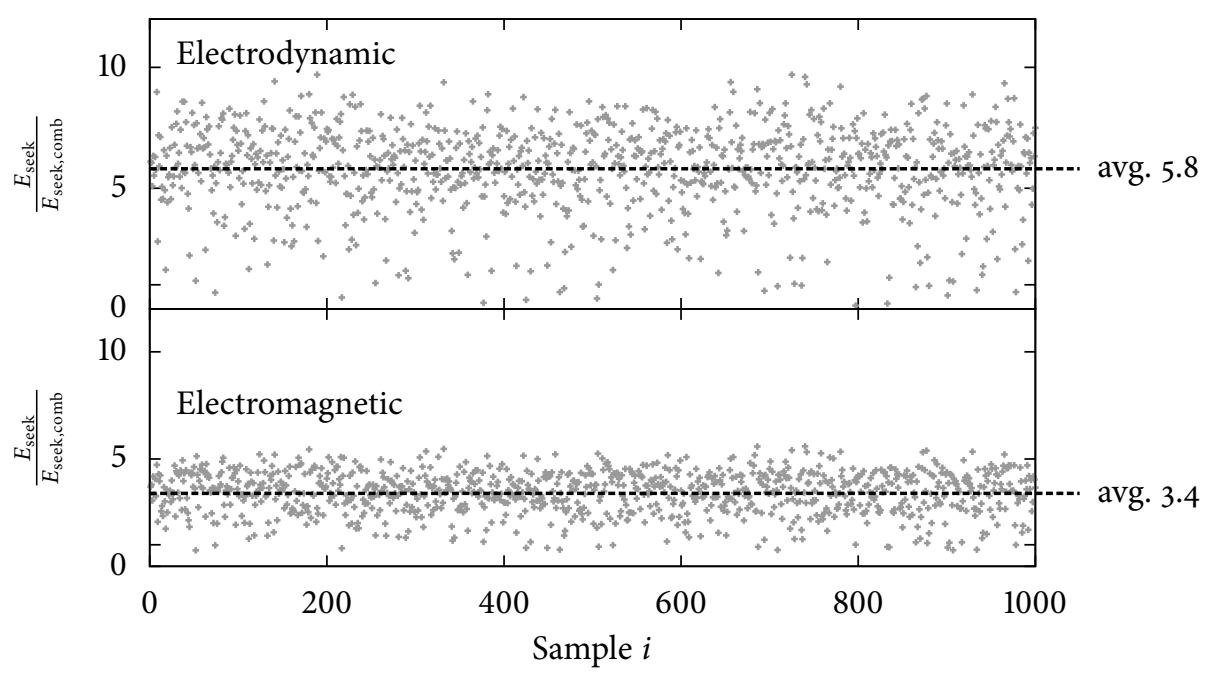

Figure 5.8 - A 'Monte Carlo' analysis of the seek energy of the electromagnetic comb-drive and electrodynamic actuator relative to the seek energy of the electrostatic comb-drive. Start and end displacements, $x_{0}$ and $x_{1}$ respectively, were randomly picked in the range $[-50 \mu \mathrm{m}, 50 \mu \mathrm{m}]$; the initial and final velocities, $v_{0}$ and $v_{1}$ respectively, were randomly picked in the range $\left[0, v_{\text {write }}\right]$.

The electrostatic comb-drive is the most energy efficient for all three operation modes. Staying at a certain displacement or moving at read speed costs roughly the same amount of energy for the electromagnetic comb-drive and electrodynamic actuator. This is explained by the fact that the dissipation by $R_{\mathrm{p}}$ or $R_{\text {coil }}$ dominates the energy cost of staying put and moving at read speed. Therefore, a good figureof-merit is the dissipated power at maximum current or voltage, $i_{\max }^{2} R_{\text {coil }}$ for the magnetic scanners, and $V_{\max }^{2} R_{\mathrm{p}}^{-1}$ for the electrostatic comb-drive. This figure-ofmerit is 13 times less for the electrostatic comb-drive than for the magnetic scanners, corresponding to the difference in $p_{\text {stay }}$ and $E_{\text {read }}$ seen in Figure 5.5 and Figure 5.6.

The energy cost difference for seek operations is less than the difference for the other operations. Still the dissipation by $R_{\mathrm{p}}$ or $R_{\text {coil }}$ is an important factor, but there is also a significant contribution from the energy cost of charging the capacitance or coils.

During normal operation of the scanner, the $y$ actuator will most often be standing still at a certain displacement, while the $x$ actuator is moving at read or write speed. Moreover, the energy cost of standing still for just $1 \mathrm{~ms}$ is comparable 
to the energy cost for a seek, and $E_{\text {seek }}$ is an order of magnitude smaller than $E_{\text {read }}$. Therefore, the total energy cost is determined chiefly by $E_{\text {stay }}$ and $E_{\text {read }}$. Therefore, the best way of reducing the energy cost would be to reduce $i_{\max }^{2} R_{\text {coil }}$ or $V_{\max }^{2} R_{\mathrm{p}}^{-1}$.

\subsection{Conclusion}

The energy cost and seek time equations for comb-drive scanners and electrodynamic scanners are summarized in Table 5.4. The hypothetical electrostatic and electromagnetic comb-drive scanners, and electrodynamic scanner that are designed to have the same force at $x=0$, have approximately equal seek performances. The electrostatic comb-drive requires an order of magnitude less energy for standing still at a certain displacement and for moving at constant read or write speed, compared with the electromagnetic comb-drive and the electrodynamic actuator, whose energy cost is roughly equal. On average, seek operations with the electrostatic comb-drive cost 3.4 or 5.8 times less energy than with the electromagnetic comb-drive or the electrodynamic actuator, respectively. The seek energy cost $E_{\text {seek }}$ is comparable to standing still for $1 \mathrm{~ms}$, and $E_{\text {seek }}$ is an order of magnitude smaller than $E_{\text {read }}$. The energy cost of operating the scanner, is determined chiefly by $E_{\text {stay }}$ and $E_{\text {read }}$, which are dominated by the dissipation by the leakage resistor, $R_{\mathrm{p}}$, parallel to the comb drive, or by the series resistance, $R_{\text {coil }}$, of the coils. Therefore, a good figure-of-merit is $i_{\max }^{2} R_{\text {coil }}$ for the magnetic scanners, and $V_{\max }^{2} R_{\mathrm{p}}^{-1}$ for the electrostatic comb-drive; this figure-of-merit is 13 times less for the electrostatic comb-drive than for the magnetic scanners. From an energy point of view, the electrostatic scanner is significantly (4-13 times) more efficient than the magnetic designs, whereas its seek time is approximately equal. 


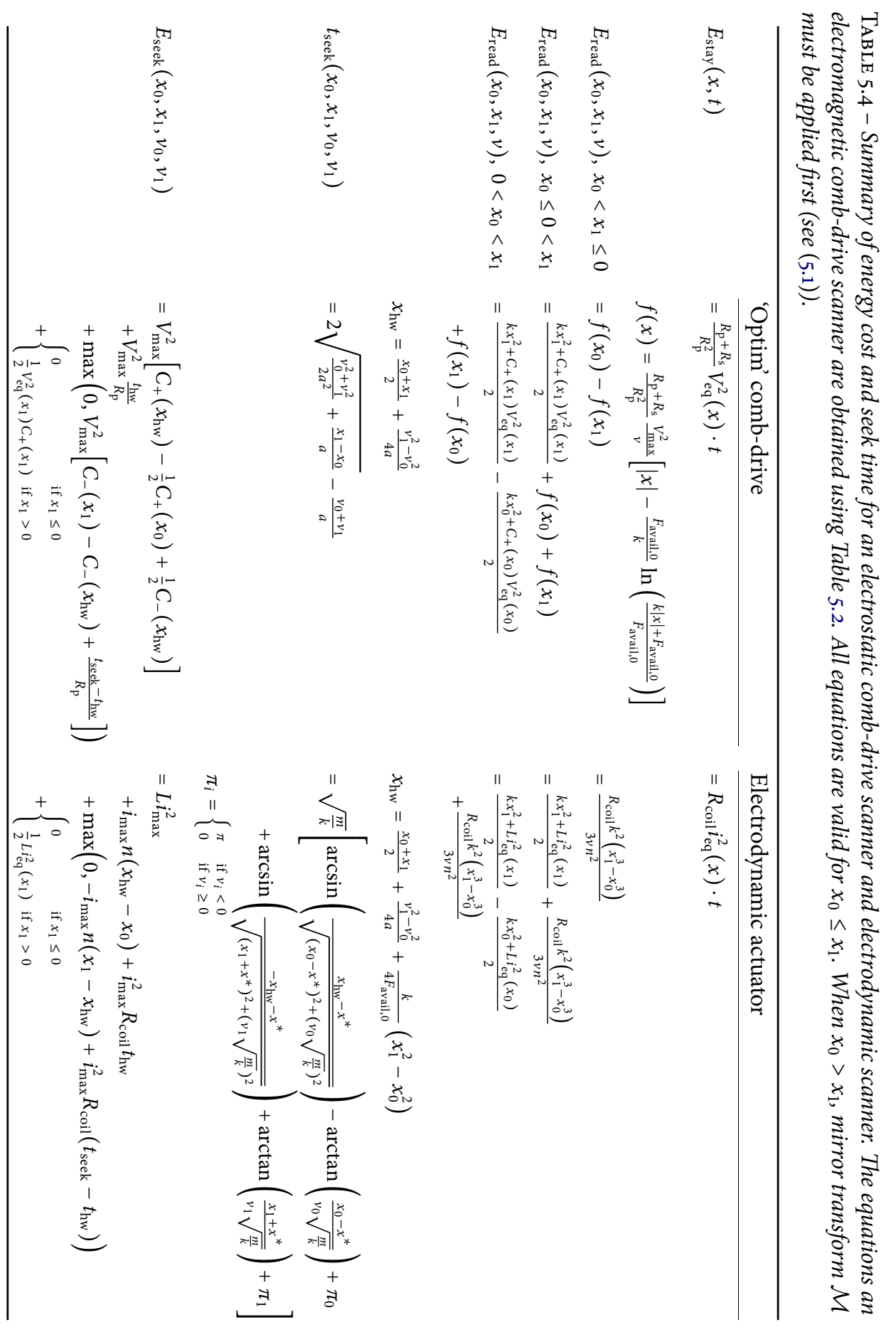




\section{Chapter 6}

\section{The Micronium - a musical MEMS instrument}

In 1997, researchers at Cornell University fabricated the world's smallest guitar, about the size of a human blood cell (Cornell University Science News, 1997). Two years later, a micro harp was reported by Carr et al. (1999). In 2003, laser light was used to strum the 'strings' of a nanoguitar (Steele, 2003). However, no human has heard the sound of these instruments; the strings vibrate at frequencies on the order of tenths of megahertz.

This chapter describes the 'micronium', a musical MEMS instrument, consisting of micromechanical mass-spring resonators that vibrate at audible frequencies. The resonators are 'plucked' using electrostatic comb-drive actuators (see Figure 6.1). The resonators generate real sound, however human ears are not sensitive enough. The MEMS flow sensor, or 'microphone' for MEMS structures (Yntema et al., 2010), developed in our group has not yet successfully been applied to listen to the generated sound. The signal-to-noise ratio of this flow sensor is expected to be low, due to the instrument residing in vacuum. Instead, the instrument's vibrations are sensed by capacitive displacement sensors using comb structures as sensing elements. The measured capacitance change is amplified to drive a loudspeaker, effectively amplifying the resonator motion 10 ooo times to the motion of the loudspeaker.

In the following, the design, fabrication and characterisation of the instrument will be discussed. The chapter will end with a discussion of the 'micronium project', that started with a second-year bachelor student project.

This chapter is an extensive elaboration of J. B. C. Engelen, H. de Boer, J. G. Beekman, A. J. Been, G. A. Folkertsma, L. C. Fortgens, D. B. de Graaf, S. Vocke, L. A. Woldering, L. Abelmann, and M. C. Elwenspoek, "A musical instrument in MEMS", presented at the $21^{\text {st }}$ Micromechanics and Micro systems Europe Workshop (MME 2010), Enschede, the Netherlands, 26-29 Sept. 2010, and has been submitted for publication. 


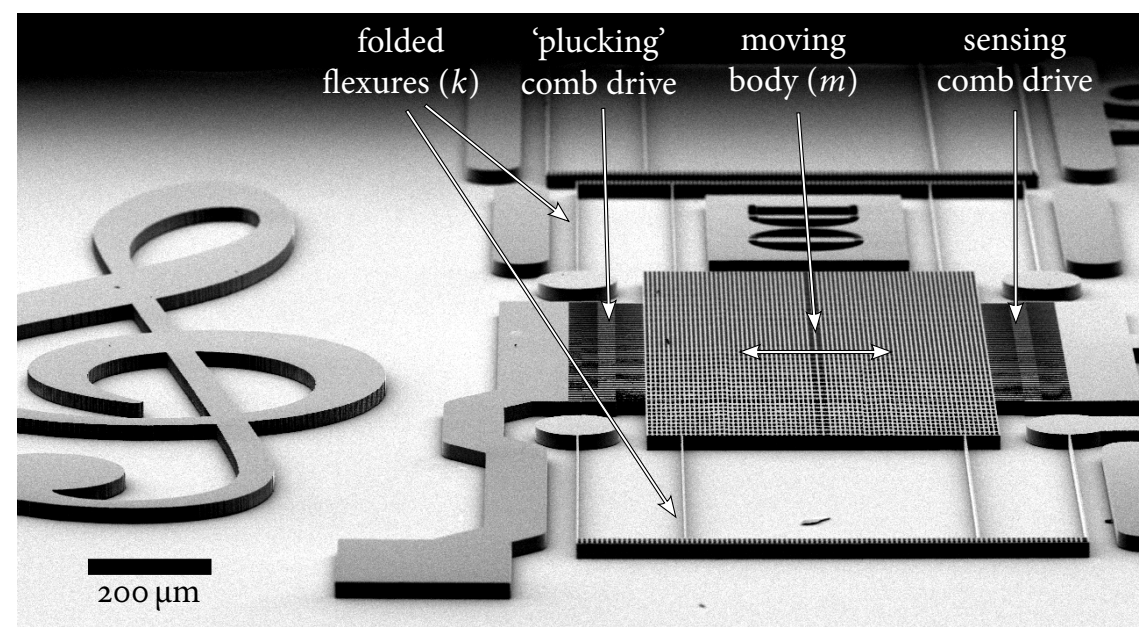

FIGURE 6.1 - Scanning electron micrograph of a resonator at audible frequency. The perforated structure is the moving body (mass $m$ ), suspended by folded flexures on both sides. The mass is 'plucked' by a comb drive on one side, and the mass's displacement is measured by a comb drive on the other side.

\subsection{Theory and design}

Our instrument consists of individual resonators for each note, similar to a xylophone. A resonator, shown in Figure 6.1, is a mass that is suspended by folded flexure springs, such that the mass is restricted to move in one direction. The resonance frequency depends on the mass $m$ and the spring stiffness $k$ of the folded flexure suspension; by adjusting the length of the springs and the width of the mass, each resonator is designed to resonate at a specific note frequency within a C-major scale (all the white keys on a piano). Each resonator is actuated by a comb drive on one side of the mass, slowly pulling the mass to one side and then releasing it so it starts oscillating. The comb drive on the other side of the mass is then used to measure the displacement by measuring the comb-drive capacitance. The measured change in capacitance is amplified and is used to drive a loudspeaker to make the resonator vibration audible to humans.

\subsubsection{Resonator theory}

A resonator behaves according to the well known differential equation

$$
m \frac{\mathrm{d}^{2} x}{\mathrm{~d} t^{2}}+\gamma \frac{\mathrm{d} x}{\mathrm{~d} t}+k x=F_{\mathrm{comb}}
$$

where $m$ is the mass of the resonator, $x$ the displacement, $\gamma$ the coefficient of viscous damping by air, $k$ the suspension spring constant, and $F_{\text {comb }}$ the comb-drive force. 
The solution of (6.1) is the expected comb-drive displacement $x$ after excitation,

$$
x(t)=A e^{-\alpha t} \cos \left(2 \pi f_{1} t\right),
$$

with amplitude $A$, and damping $\alpha=\gamma / 2 m$. The free resonance frequency $f_{1}$ for an underdamped system is

$$
f_{1}=\frac{1}{2 \pi} \sqrt{\frac{k}{m}-\alpha^{2}} \approx f_{0}\left(1-\frac{1}{8 Q^{2}}\right),
$$

with $2 \pi f_{0}=\sqrt{k / m}$, and $Q=\pi f_{0} / \alpha$. The approximation is correct for low damping. Tailoring the mass and spring constant, resonators with different resonance frequencies are made. In order to obtain resonance frequencies in a range from $440 \mathrm{~Hz}$ to $1400 \mathrm{~Hz}$, large structures are needed compared with common MEMS structure sizes: the spring lengths range from $0.5 \mathrm{~mm}$ to $0.9 \mathrm{~mm}$ and the mass areas range from $0.6 \mathrm{~mm}^{2}$ to $1 \mathrm{~mm}^{2}$.

The duration of a note after being excited/struck, is proportional to the quality factor $Q$ and inversely proportional to the resonance frequency. A piano string has a quality factor much larger than 1000. It is hard to obtain high quality factors for MEMS structures because of unfavorable scaling laws. For a mass-spring-dampersystem resonator, the quality factor equals

$$
Q=\frac{\sqrt{m k}}{\gamma} .
$$

When the dimensions of the mass are scaled with an arbitrary factor $l$, the mass scales cubically, $m \propto l^{3}$. The spring constant should scale proportionally to the mass in order to maintain the same resonance frequency, hence $k \propto m$. The viscous damping is proportional to the area of the mass, $\gamma \propto l^{2}$. Consequently, the quality factor scales with $l$; reducing the dimensions of the system results in a proportionally reduced Q-factor. In general, at equal resonance frequencies, a smaller mass-spring oscillator will experience more damping and will sound a shorter note than a larger oscillator. To solve this issue of small MEMS instruments, we decrease the air damping $\gamma$, by placing the instrument in a vacuum chamber. Ambient pressures below 2 mbar significantly increase the Q-factor, because the mean free path of the air molecules becomes larger than the gap sizes of our resonators (Okada et al., 2009).

\subsubsection{Instrument design}

The instrument design consists of 24 resonators at 12 different frequencies, two resonators for each frequency, spread across four $7 \mathrm{~mm} \times 7 \mathrm{~mm}$ chips. The instrument is designed such that the notes form part of a $\mathrm{C}$-major diatonic scale (the white keys on a piano: C, D, E, F, G, A and B). Note A of the fourth octave on a modern 88 -key piano, called $\mathrm{A} 4$, is tuned to $440 \mathrm{~Hz}$ (Young, 1939). The note frequencies follow from

$$
f(n)=2^{\frac{n}{12}} \times 440 \mathrm{~Hz},
$$


with

$$
n \in\{0,2,3,5,7,8,10,12,14,15,17,19\}
$$

indicating the number of semitones ${ }^{*}$ above A4 (Young, 1939).

The general layout of an instrument chip is shown in Figure 6.2. Both the suspension spring stiffness $k$ and the moving body mass $m$ of the resonators are adjusted to obtain the desired resonance frequencies. The spring stiffness of the folded flexure suspension equals (Legtenberg et al., 1996)

$$
k=\frac{2 E h b^{3}}{L^{3}}
$$

where $E$ is the effective Young's modulus of silicon, $h$ the spring height, $b$ the spring width, and $L$ the spring length. The effective mass of the resonator $m_{\mathrm{eff}}$ is equal to the moving body mass, including comb-drive fingers, plus the folded flexure truss mass, neglecting the mass of the spring beams. Because the folded flexure trusses move only half the distance of the moving body, only half their mass contributes to the effective mass. The moving body and trusses need to be perforated because of the used fabrication process. The perforation consists of $5 \mu \mathrm{m} \times 5 \mu \mathrm{m}$ squares with $3 \mu \mathrm{m}$ silicon beams in between. This results in an area reduction $R_{\text {perf }}$ of approximately 0.6 ,

$$
R_{\text {perf }} \approx \frac{8^{2}-5^{2}}{8^{2}} \approx 0.6
$$

The moving body has height $h$, width $W$, and length $B$. Referring to Figure 6.2, $B=1.2 \mathrm{~mm}$ is fixed for all resonators, and $W$ is adjusted. The area of the perforated trusses $A_{\text {truss }}$ is the product of the width and length, $32 \mu \mathrm{m}$ and $W+333 \mu \mathrm{m}$, respectively. The total area $A_{\text {fingers }}$ of the comb-drive fingers on both sides of the resonator equals $3.84 \times 10^{-8} \mathrm{~m}^{2}$. We find for the effective mass,

$$
m_{\mathrm{eff}}=\rho_{\mathrm{Si}} h \cdot\left[A_{\text {fingers }}+R_{\text {perf }}\left(W B+A_{\text {truss }}\right)\right] .
$$

The layout of the instruments chips is shown in Figure 6.2. The $50 \mu \mathrm{m}$ thick device layer of the silicon-on-insulator wafer used in the fabrication process, determines height $h$ of the comb drives, springs and masses. The smallest trench that can be etched is $3 \mu \mathrm{m}$ wide, defining the gap $g$ between comb-drive fingers. Each comb drive contains 60 finger pairs, and the initial overlap $x_{0}$ equals $30 \mu \mathrm{m}$. The spring width $b$ is $3 \mu \mathrm{m}$, which is the smallest beam width that can be fabricated reliably. The mass width $W$ and spring length $L$ of the resonators are listed in Table 6.1.

\subsubsection{Capacitive read-out}

By measuring the capacitance of a comb drive connected to the mass, the displacement of the mass is determined. The measured capacitance is used as the audio

\footnotetext{
${ }^{*} \mathrm{~A}$ semitone is the musical interval between two adjacent notes on a piano, for example between $\mathrm{C}$ and $C^{\sharp}$.
} 


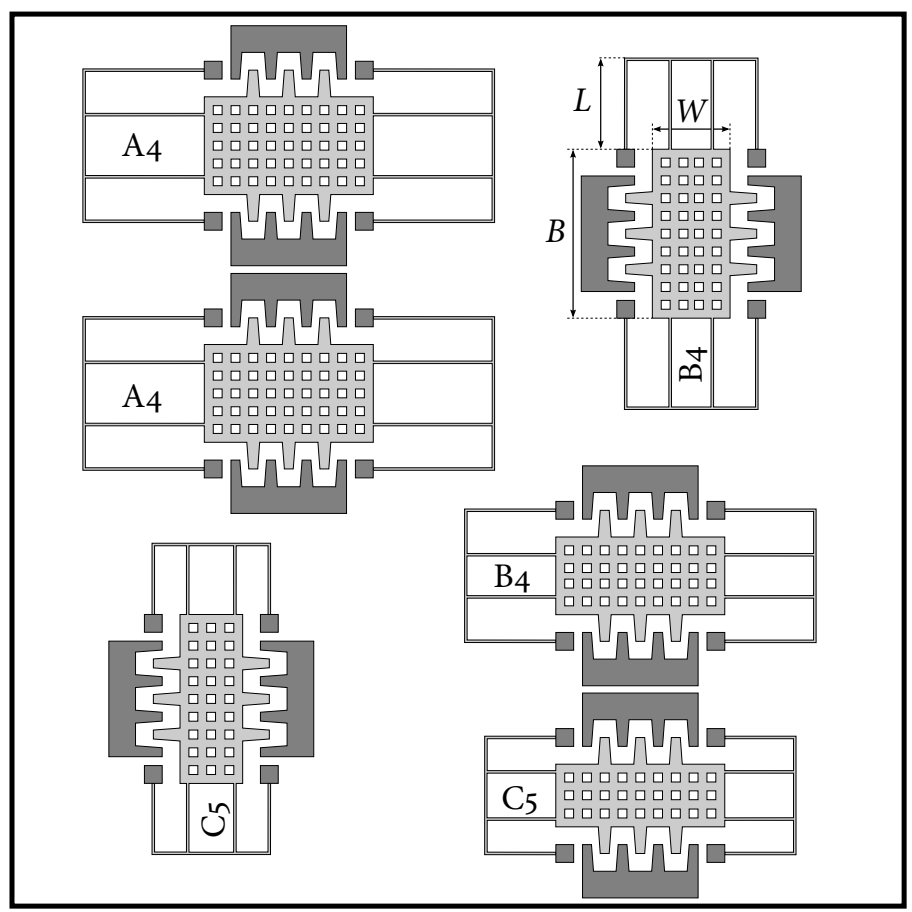

FIGURE 6.2 - Layout of the $7 \mathrm{~mm} \times 7 \mathrm{~mm}$ instrument chips (not drawn to scale). Six resonators fit on one chip. Four chip types are designed to fit twelve different note frequencies: chips $c_{1}, c_{2}, c_{3}$ and $c_{4}$ contain notes $\left(A_{4}, B_{4}, C_{5}\right),\left(D_{5}, E_{5}, F_{5}\right),\left(G_{5}, A_{5}, B_{5}\right)$ and (C6,D6,E6), respectively. The comb drives and length $B$ are equal for all resonators, only the spring length $L$ and mass width $W$ are varied to tune the resonance frequencies.

signal. Besides ease of fabrication, the use of a comb drive allows us to adjust the timbre of the note, by modifying the comb-drive finger shape. For straight comb-drive fingers, the capacitance is proportional to the displacement. However, the capacitance of a comb drive with tapered fingers depends non-linearly on the displacement $x$,

$$
C_{\text {tapered }}=2 N \varepsilon_{0} h \frac{x+x_{0}}{g-\left(x+x_{0}\right) \tan \theta},
$$

where $N$ is the number of fingers, $h$ is the comb-drive height, $x_{0}$ and $g$ are the initial overlap and gap between fingers respectively, and $\theta$ the angle of the tapering (Mohr et al., 1992). This non-linearity gives rise to higher harmonics in the audio signal, resulting in a more interesting tone. The upper bound on the angle $\theta$ is set by fabrication limits; $\theta=0.72^{\circ}$ for our designs, such that the reduced gap between fingers can still be reliably fabricated. The angle is small because of the relatively large overlap $x_{0}$ between combs. Figure 6.3 shows the Fourier transform of the 
TABLE 6.1 - Design target frequencies $f_{0}$ of the instrument notes and the resulting design parameters for the spring length $L$ and moving body size $W$. The parameters are rounded to integer micrometers, therefore the target frequencies deviate $( \pm 0.05 \%$ or less) from the ideal frequencies $f(n)$ given by (6.3).

\begin{tabular}{|c|c|c|c|}
\hline Note & $f_{0}(\mathrm{~Hz})$ & $W(\mu \mathrm{m})$ & $L(\mu \mathrm{m})$ \\
\hline $\mathrm{A}_{4}$ & 440.1 & 846 & 910 \\
\hline $\mathrm{B}_{4}$ & 493.9 & 845 & 843 \\
\hline $\mathrm{C}_{5}$ & 523.3 & 702 & 859 \\
\hline $\mathrm{D}_{5}$ & 587.4 & 700 & 796 \\
\hline $\mathrm{E}_{5}$ & 659.3 & 700 & 737 \\
\hline $\mathrm{F}_{5}$ & 698.7 & 700 & 709 \\
\hline $\mathrm{G}_{5}$ & 784.0 & 551 & 706 \\
\hline $\mathrm{A}_{5}$ & 879.7 & 551 & 654 \\
\hline $\mathrm{B}_{5}$ & 987.9 & 552 & 605 \\
\hline C6 & 1047.0 & 552 & 582 \\
\hline D6 & 1174.7 & 552 & 539 \\
\hline E6 & 1318.8 & 552 & 499 \\
\hline
\end{tabular}

simulated capacitance for straight and tapered fingers using (6.2) for displacement $x$. Besides the fundamental, higher harmonics are present in the capacitance of the tapered fingers. The relative amplitude of the higher harmonics depends on the displacement amplitude $A$.

\subsubsection{Tuning}

Two methods of tuning the resonance frequency are softening the suspension stiffness by lowering its Young's modulus by heat, and reducing the effective spring constant by applying a DC offset voltage on the comb drives that induces a negative 'electrostatic stiffness'.

Thermally tuning the resonance frequency of resonators takes advantage of the temperature dependence of the Young's modulus and the thermal expansion of silicon (Remtema and Lin, 2001; Syms, 1998). Heating the springs by running an electrical current through them, the Young's modulus is decreased (Cho, 2009), resulting in a lower resonance frequency. Silicon expands when heated, which can induce compressive or tensile strain, depending on the geometry, leading to a change in resonance frequency (Syms, 1998). Initial experiments on the micronium's resonators indicate that the resonance frequency can be decreased by $5 \%$ in air. Cooling by convection of the surrounding air, and therefore the tuning result, depends on the ambient pressure (Syms, 1998). Currently, the total measurement setup is unsuited for electrothermal tuning experiments, and no experiments have been done at 1 mbar yet.

An electrostatic spring stiffness can be created by applying a constant offset voltage on the comb drives. The effective spring constant $k_{\text {eff }}$ is equal to the derivative of the total force on the mass with respect to $x$. When a constant voltage is applied 


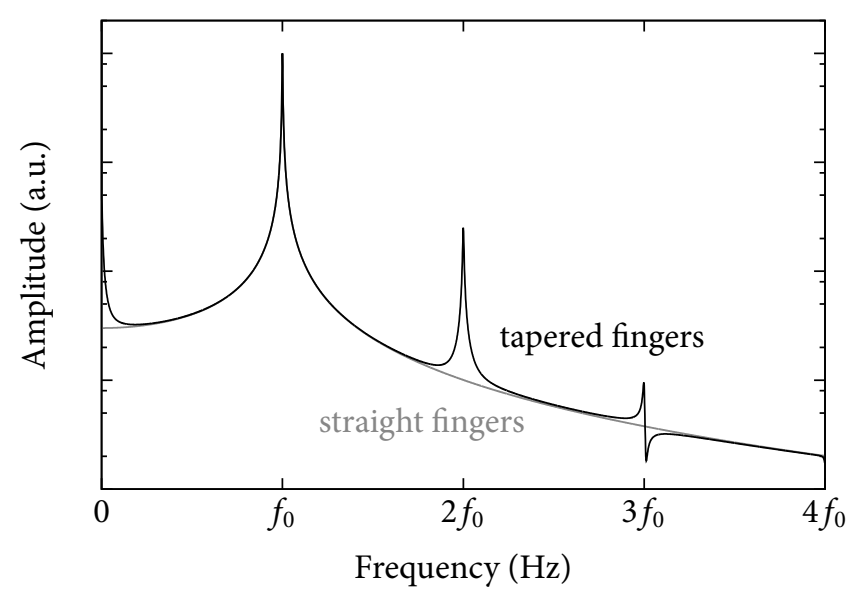

FIGURE 6.3 - The Fourier transform of the simulated capacitance of comb drives with straight and tapered fingers for an exponentially decaying sinusoid displacement. The non-linear capacitance versus displacement of tapered fingers gives rise to higher harmonics proportional to the sinusoid displacement amplitude.

on one of the comb drives, the effective spring constant is equal to

$$
k_{\mathrm{eff}}=\frac{\partial F}{\partial x}=k-\frac{1}{2} \frac{\partial^{2} C}{\partial x^{2}} V^{2} .
$$

For straight comb-drive fingers, $\frac{\partial^{2} C}{\partial x^{2}}$ vanishes. But the tapered finger shape used in our instrument results in an electrostatic force that is a function of position, and the effective spring constant becomes a function of applied voltage $V$ and $x$,

$$
k_{\text {eff }}=k-\varepsilon_{0} N h V^{2}\left(\frac{2 \tan (\theta)}{\left(g-\tan (\theta)\left(x+x_{0}\right)\right)^{2}}+\frac{2 \tan ^{2}(\theta)\left(x+x_{0}\right)}{\left(g-\tan (\theta)\left(x+x_{0}\right)\right)^{3}}\right) .
$$

At a constant voltage, the electrostatic force increases for increasing $x$, corresponding to a negative non-linear spring constant, decreasing the resonance frequency of the resonator. Note that, because the effective spring stiffness depends on $x$, the exact frequency decrease depends on the amplitude of motion. More importantly, because $k$ is larger for resonators at higher frequencies than for resonators at lower frequencies, and because the electrostatic spring stiffness change is additive, the relative frequency decrease at equal offset voltage will be lower for resonators at high frequencies.

\subsection{Experimental details and results}

The instrument chips are fabricated from one (1 o o) single-crystal highly borondoped silicon-on-insulator wafer, with a $50 \mu \mathrm{m}$ thick device layer and an oxide 


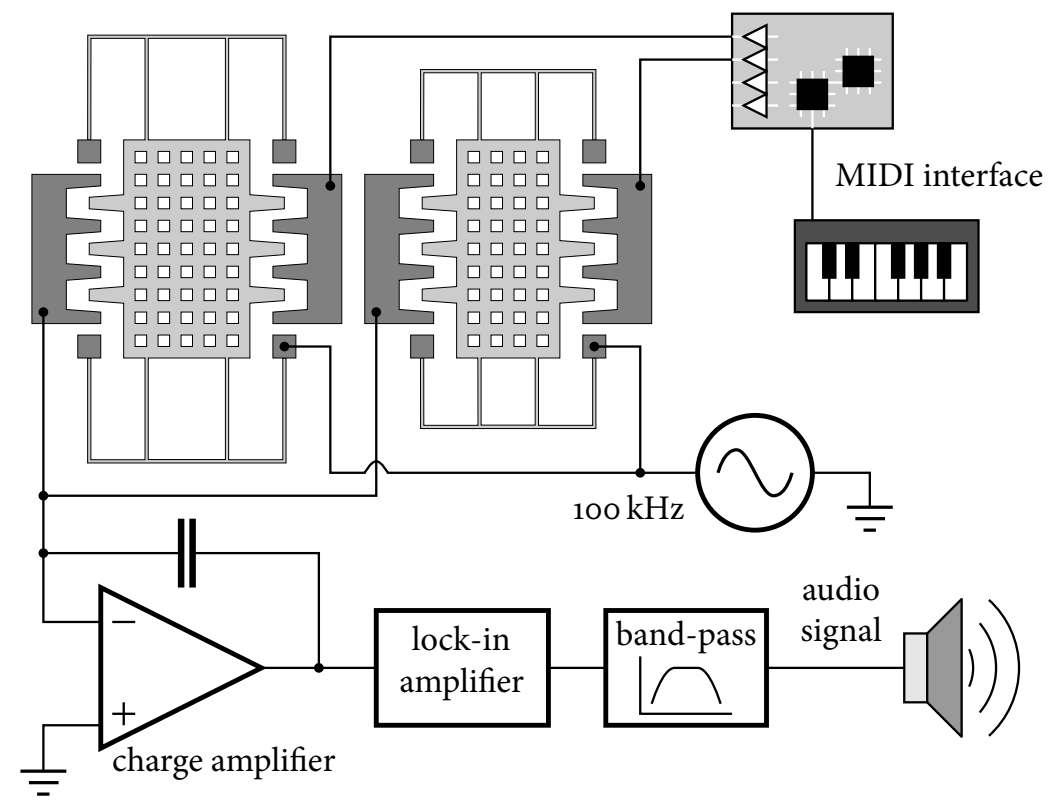

FIGURE 6.4 - Simplified drawing of the used measurement setup. The displacement of a resonator is measured from the comb-drive capacitance, using a charge amplifier circuit and lock-in amplifier. In total, 12 resonators are connected in parallel to one charge amplifier circuit (only two resonators are drawn). The resonators are actuated by high-voltage amplifiers that are controlled through a MIDI interface.

thickness of $3 \mu \mathrm{m}$. The structures are made by deep reactive-ion etching (Jansen et al., 2009; Laermer and Schilp, 1994), after which the (movable) structures are released by HF vapour phase (VHF) etching (Holmes and Snell, 1966) of the oxide layer. The VHF etching is isotropic, and also etches oxide under the structures. The VHF etch-time is tuned such that thin beams and perforated structures are released, and large structures are not. More details about the fabrication process can be found in Appendix C.

The measurement setup, partly shown in Figure 6.4, consists of two charge amplifiers (Wiegerink et al., 2007), each sensing 12 resonators in parallel, two lockin amplifiers, and an additional amplifier with band-pass filter. The output of the band-pass filter is used as audio signal and is connected to an amplifier with a loudspeaker, or to a microphone input of a PC soundcard for measurements. The sine waves used for sensing the capacitance changes with the lock-in amplifiers have a frequency around $90 \mathrm{kHz}$ with an amplitude around $400 \mathrm{mV}$; the precise values are chosen depending on the situation to reduce the noise and external disturbances. The resonators are actuated by a programmable microcontroller that controls D/Aconverters followed by high-voltage amplifiers. At rest, the applied voltage is zero. 


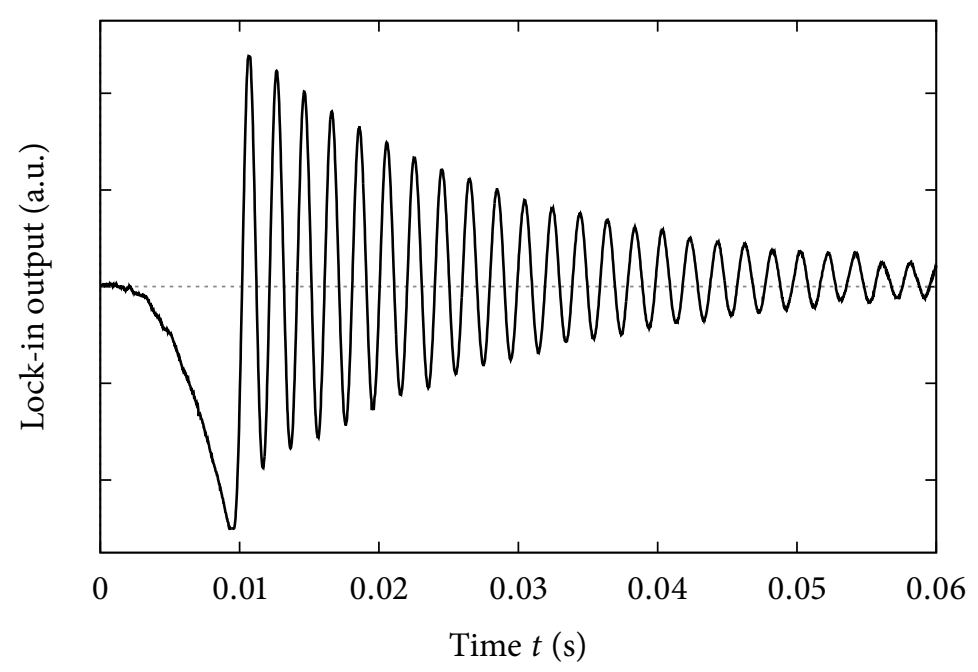

FIGURE 6.5 - A recorded lock-in output signal of an 'A4 resonator' at an ambient pressure of $37 \mathrm{mbar}$ upon pressing the keyboard key. The negative ramp at the start shows the 'plucking' of the note, pulling the resonator back before it is released (remember that the lock-in output is approximately equal to the resonator displacement). The actuation voltage ramp stops just before $10 \mathrm{~ms}$, and the resonator starts oscillating according to (6.2).

A note is 'plucked' by ramping the applied voltage in several milliseconds up to the actuation voltage $V_{\text {act }}$, and subsequently rapidly reducing the applied voltage back to zero. The ramp prevents sounding a note upon the increase of the applied voltage. This 'plucking ramp' simulates manually pulling back the resonator and releasing it, similar to plucking a string, as can be seen at the start of the audio signal in Figure 6.5. The height of the actuation voltage $V_{\text {act }}$ is determined by the velocity parameter received in the MIDI messages from the MIDI keyboard; the harder a key is pressed on the keyboard, the larger $V_{\text {act }}$ and the louder the note will sound. The value for $V_{\text {act }}$ is maximally $29 \mathrm{~V}$.

Figure 6.5 shows a recorded lock-in amplifier output signal of an 'A4 resonator'. As expected from (6.2) and (6.4), the signal is a decaying sine wave. The tone sounds very pure, and is similar to the sound of a xylophone. The Fourier transform of the audio signal from an 'A4 resonator' is shown in Figure 6.6. There is only one sharp peak visible, at $506 \mathrm{~Hz}$. There signal contains no higher harmonics.

The measured resonance frequencies of 83 resonators from 14 chips are shown in Figure 6.8. For each design frequency, there is a large spread in measured frequencies. Almost all resonators resonate at a higher frequency than their design predicts (up to 5 semitones higher).

The damping $\alpha$ and quality factor $Q$ are determined by fitting an exponentially 


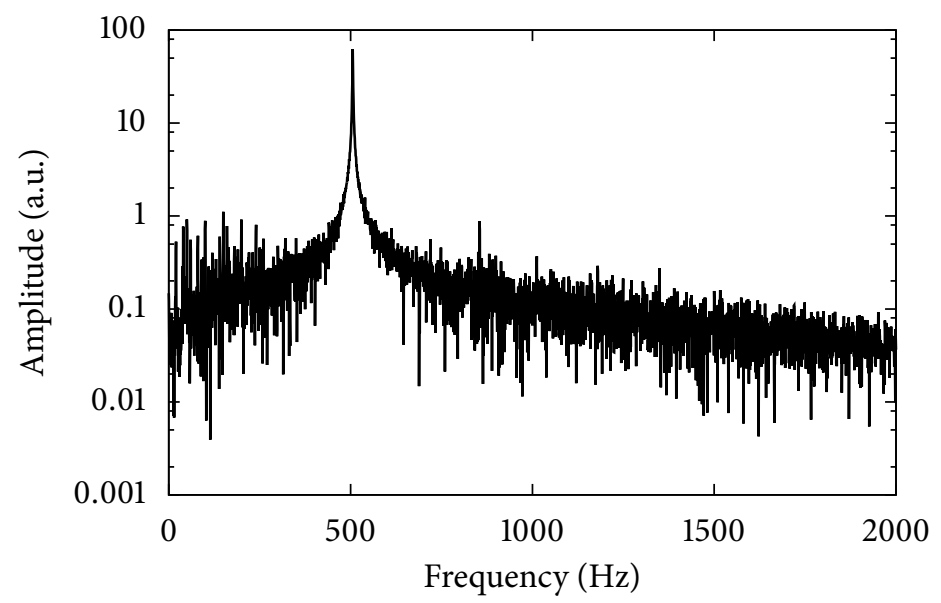

FIGURE 6.6 - The Fourier transform of the audio signal of an 'A4 resonator', showing one resonance peak $(506 \mathrm{~Hz})$. There is no visible effect of the non-linear capacitance versus displacement of the tapered comb-drive fingers.

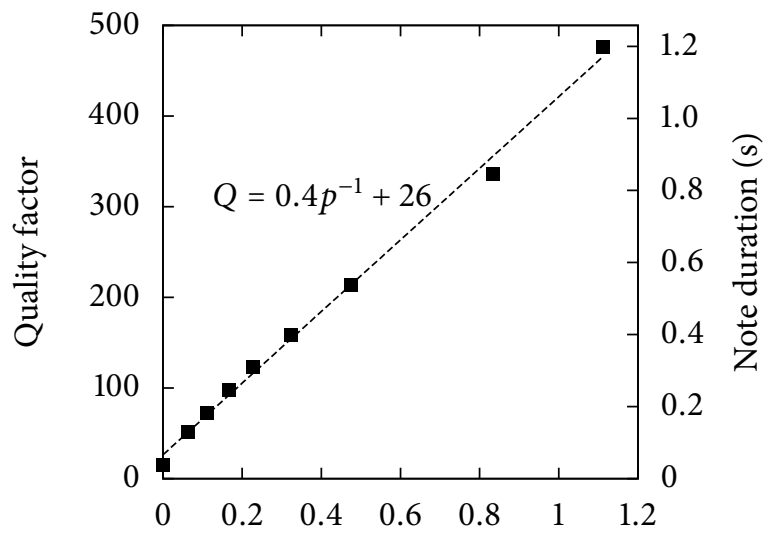

Reciprocal pressure $p^{-1}\left(\mathrm{mbar}^{-1}\right)$

FIGURE 6.7 - Quality factor measurements of an 'A4 resonator' $(506 \mathrm{~Hz})$ at different pressures. The quality factors are obtained from fitting an exponentially decaying sinusoid to the measured audio signal. The dashed line fits the data with $Q(p)=a p^{-1}+b$, where $a=0.40$ bar \pm 0.01 bar and $b=26 \pm 5$. The right $y$-axis indicates the apparent note duration $\left(4 \alpha^{-1}\right)$. 


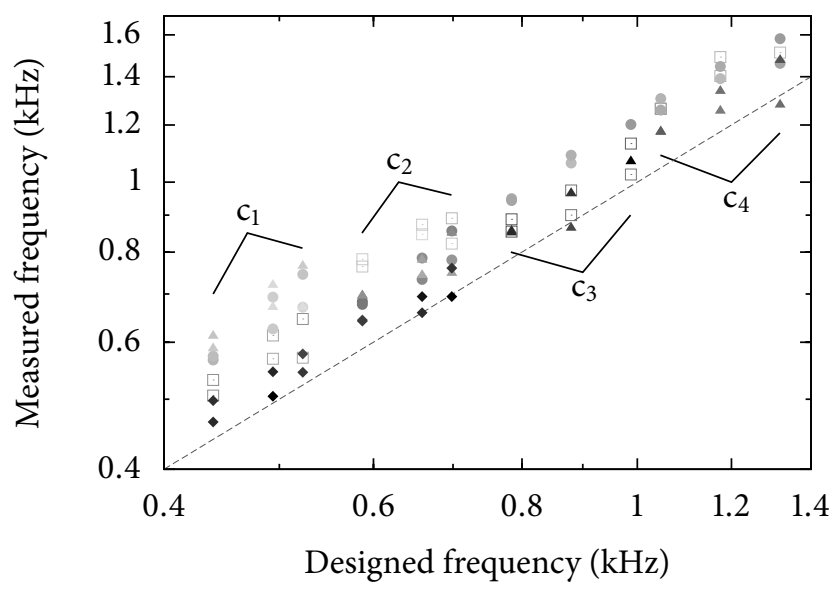

FIGURE 6.8 - The measured resonance frequencies of 83 resonators versus their designed resonance frequencies, all from the same silicon wafer. Note that the $x$ and $y$ axes are logarithmic. The dashed line shows when the measured frequency equals the design. Within a group $c_{i}$, equal symbols are used for resonators from the same chip. The open squares represent the 24 resonators used in the micronium. The grayscale value indicates the distance to the centre of the wafer for each resonator; resonators with darker points lie closer to the centre, see Figure 6.9.

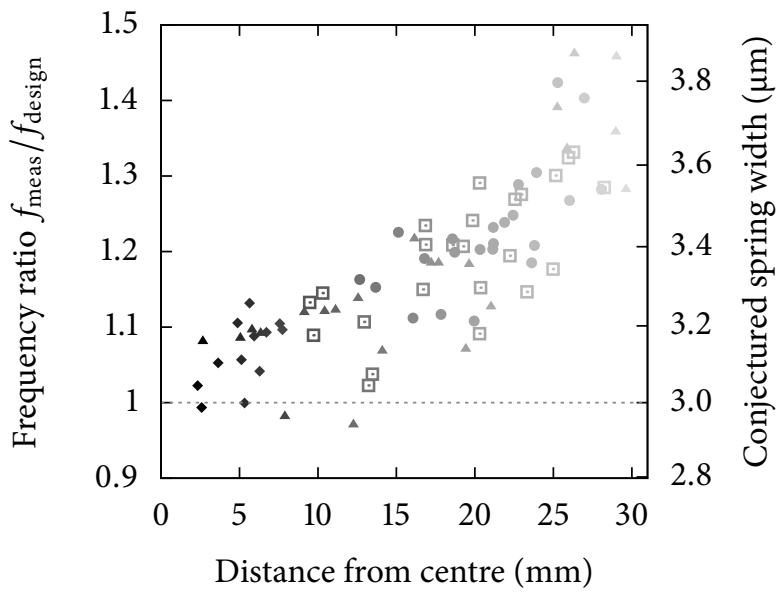

FIGURE 6.9 - The ratio of measured and designed resonance frequencies from Figure 6.8, as a function of distance from the centre of the wafer. If the frequency increase is only due to an increased spring stiffness, the $y$ axis indicates the spring width corresponding to the frequency ratio. 


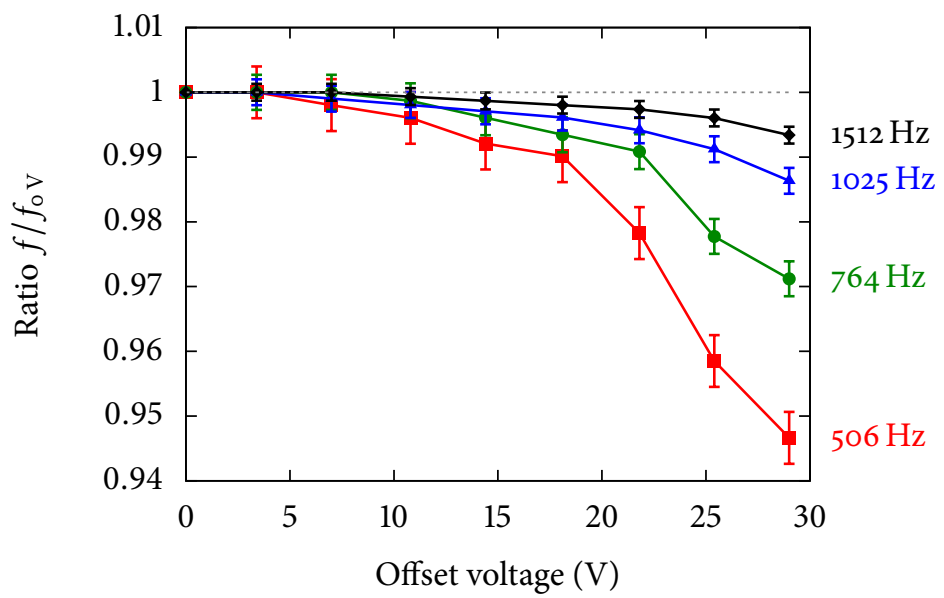

FIGURE 6.10 - The ratio between the resonance frequency with an applied offset voltage on the plucking comb-drive and the resonance frequency without offset voltage, for four resonators. The resonance frequency without offset voltage is indicated on the right next to each graph. The lines connecting the measurement points are guides to the eye.

decaying sinusoid to the audio signal; Figure 6.7 shows the quality factor of an 'A4 resonator' for different ambient pressures. The apparent note duration can be approximated by $4 \alpha^{-1}$, and is proportional to the quality factor. Without the vacuum chamber ( $p=1$ bar), only a very brief oscillation of less than $20 \mathrm{~ms}$ was measured. The duration of the note is greatly increased at a reduced pressure. The quality factor and note duration are proportional to the reciprocal of the ambient pressure, on the investigated range from 1000 mbar to 1 mbar. A close linear fit to the measurements can be made with $Q(p)=a p^{-1}+b$, where $a=0.40$ bar \pm 0.01 bar and $b=26 \pm 5$.

Figure 6.9 shows the data from Figure 6.8, plotting the ratio between the measured and designed frequency as a function of distance from the centre of the wafer. On average the resonance frequency is $18 \%$ higher than expected. Resonators that are closer to the centre of the wafer deviate less from the design than resonators that lie farther from the centre. The increase in frequency may be explained by a larger than designed spring width. The right $y$-axis in Figure 6.9 indicates the spring width corresponding to the frequency ratio on the left $x$-axis, if all other parameters are kept constant.

Tuning of the resonance frequency is measured by applying a constant offset voltage $V_{\text {offset }}$ on the plucking comb drive. The actuation signal on the plucking comb drive is modified to incorporate the offset voltage. The modified plucking signal starts at $V_{\text {offset }}$, is ramped up to $29 \mathrm{~V}$ in several milliseconds, is rapidly decreased to zero, and finally after $11 \mathrm{~ms}$ it is set back to $V_{\text {offset }}$. No offset is applied to the 
sensing comb-drive. Figure 6.10 shows measurements of the resonance frequencies of four resonators as a function of the applied offset voltage on the plucking combdrive, relative to the resonance frequency without offset voltage. Applying an offset decreases the resonance frequency; a decrease of more than $5 \%$ is obtained for the $506 \mathrm{~Hz}$ resonator at $29 \mathrm{~V}$. The relative decrease depends on the original resonance frequency: the higher the resonance frequency, the smaller the relative decrease.

\subsection{Discussion}

The micronium has a very clean and pure sound, and sounds similar to a xylophone. Because the resonator frequencies deviate from their design, more notes can be played than the designed 12 notes ( 24 resonators, indicated by open squares in Figure 6.8). By carefully selecting the closest note for each resonator frequency, the instrument can play 16 notes spanning almost two octaves ( $\mathrm{C}$ to $\mathrm{A}$ ) that are reasonably in-tune without tuning. The 8 unused resonators are either close to notes that are already represented by the 16 chosen resonators, or are too much out-of-tune. On the instrument's range from $\mathrm{C}$ to $\mathrm{A}$, six notes are missing (low octave: F, $F^{\sharp}$; high octave: $C^{\sharp}, D^{\sharp}, F, G^{\sharp}$ ). The available notes allow many tunes to be played on the micronium.

The Fourier spectrum of the audio signal of one resonator shows one large and sharp peak. Although the capacitive read-out of the resonator displacement is non-linear, the displacement amplitude is not large enough for higher harmonics to appear in the audio signal. The resulting sound of the instrument is therefore very clean and pure.

The note duration is proportional to the reciprocal of the ambient pressure on the investigated range, in qualitative agreement with Okada et al. (2009, eq. (7)). A piano-like sustain pedal could be simulated by controlling the pressure from 10 mbar to 1 mbar or lower.

Because of intrinsic uncertainties in fabrication, there is a large uncertainty in the resonance frequencies after fabrication. Resonators of exactly equal design and positioned next to each other on the wafer, have resonance frequencies that differ by as much as $18 \%$. To obtain a playable instrument, either measuring or choosing a specific resonator for each note, or tuning of the resonators is necessary. The increase in resonance frequency from the designed frequency is larger for resonators that are farther from the centre of the wafer. It is likely this is caused by a larger than designed spring width, because the springs are thin and therefore the resonance frequency is most sensitive to changes in spring width (as compared to a change in mass area). The etch rate at the centre of the wafer is larger than on the periphery, providing a potential explanation for the larger spring width for resonators farther from the centre (see Figure 6.9).

Note in Figure 6.2 that the $\mathrm{A}_{4}$ resonators have the same orientation, in contrast to the $\mathrm{B}_{4}$ resonators and the $\mathrm{C}_{5}$ resonators. Resonators from the same chip with the same orientation (the first column in each group $c_{i}$ in Figure 6.8) generally show less spread than resonators from the same chip but with different orientation (the 
second and third columns in each chip type $c_{i}$ in Figure 6.8). Also, the spread in obtained resonance frequencies seems independent of the designed frequency or distance from the centre of the wafer. However, more statistical data is required to draw conclusions regarding these observations of the frequency spread.

There are several methods of tuning the resonance frequencies, but the setup only allowed tuning by applying an offset voltage on the plucking comb-drive. The tapered finger shape leads to a non-linear electrostatic spring stiffness; the effective spring stiffness with an offset voltage decreases for increasing displacement. This means that for a sustained note, the frequency should increase as the volume decreases. This effect, however, is small and has not been heard during the measurements. The larger relative decrease in frequency for low notes than for high notes, seen in Figure 6.10, is explained by the additive character of the electrostatic spring stiffness change. For the lowest note $(506 \mathrm{~Hz})$ a $5 \%$ lower frequency was obtained. But for a high note at $1512 \mathrm{~Hz}$, less than $1 \%$ change was obtained at an equal offset voltage. Because a $6 \%$ decrease in frequency corresponds to one semitone, a tuning range of $6 \%$ is desired in order to tune the resonator's frequency precisely to the nearest piano note frequency. Increasing the offset voltage for a larger frequency decrease should be done with care and is limited by sideways pull-in.

Large permanent frequency adjustments can be realised through mass finetuning performed by depositing additional material on the moving body. Another possible tuning method is to electrostatically pull on the folded-flexure trusses in the direction perpendicular to motion, similar to the 'axial tuning' proposed by Yao and MacDonald (1995). Because the trusses move inward (in the direction of the mass) when the mass is displaced, pulling the trusses outward will increase the resonance frequency. Because there is no snap-in possibility, very high voltages can be used in this method.

\subsection{Conclusion}

We successfully designed and realised a musical instrument using MEMS technology, that resonates at audible frequencies. The generated tones are decaying sine waves without higher harmonics, and sound similar to a xylophone. Viscous damping by air is relatively large for micro resonators at audible frequencies, resulting in a short tone. A vacuum around 1 mbar is required for a note duration around $1 \mathrm{~s}$. The frequencies of the fabricated notes are higher than expected ( $18 \%$ on average, but up to $46 \%)$, and there is a spread of up to 5 semitones $(\sim 34 \%)$ in obtained frequencies between resonators with identical designs. The increase is larger for resonators farther from the centre of the wafer, and is likely caused by thicker than designed springs. Tuning of the instrument notes is possible by applying a constant offset voltage on the plucking comb-drive, however more research is needed to increase the tuning range, especially for higher frequencies. 


\subsection{The Micronium project}

The creation of the micronium started with a second-year bachelor student project in 2009, called the ' $\mathbf{2} 2$ project', in which four second-year bachelor students spend eight weeks full-time on a project within one of the Electrical Engineering research groups of the University of Twente. With the desire to provide 'hands-on' experience with MEMS, we devised a project to create a musical instrument in MEMS. The students would create the mask designs and do the measurements, but cleanroom fabrication would be done by supporting staff and Ph.D. students. The used fabrication process should be simple enough to be completed within a short time to leave enough time for measurements, and was borrowed from a similar project one year before.

The students made many different instrument designs, conservative as well as exotic, and experimented with different methods to measure and amplify the generated sound. A vacuum chamber with many electrical feedthroughs was quickly crafted when it became apparent that the air damping was too large for audible notes. However, due to a low fabrication yield and underestimated problems with sticking of the resonators to the bulk, only a single, almost inaudible, measurement of a note was obtained with a duration of several milliseconds. Although the project was very successful, it left the students a few, seemingly small, steps shy of fabricating the first musical MEMS instrument.

These seemingly small steps - fabricating more reliable resonators, finishing the control circuitry, and improving the signal-to-noise ratio of the audio signal proved to be quite big steps. A very rudimentary instrument was demonstrated at the opening of the 'SmartXP lab' at the University of Twente. Other students helped to finalize the micronium, which was first demonstrated at the Micromechanics and Micro systems Europe Workshop (MME 2010) in Enschede, the Netherlands, one-and-a-half year after the start of the $\mathbf{B} 2$ project. The micronium was played live during the opening ceremony of our university's NanoLab.

Creating a MEMS instrument from scratch is too much work for an eight-week second-year bachelor student project. However, it is a very interesting and fun way to learn about MEMS, and with the acquired knowledge about MEMS instruments, it is possible to devise a smaller project in which emphasis is put on generating sound instead of a creating a complete instrument.

The micronium has been quite successful in exposing MEMS technology. It was featured on radio, in the Dutch newspaper NRC, the London metro and other printed media, and on the internet where many websites had a news item about the 'world's smallest violin' ${ }^{\dagger}$. As a measure of its popularity on the internet, the videos of the presentation at MME 2010 have been viewed over 40 thousand times on the internet (Vimeo and YouTube, search for 'micronium'). However, judging from the type of reporting websites and the categorization in other media, the interested audience is probably still mostly technically oriented.

\footnotetext{
${ }^{\dagger}$ Should our curriculum include media training?
} 


\subsection{Acknowledgements}

I received a lot of help in realising the micronium. Many people have been involved and contributed to its success. First of all, the B2 project students are Jethro Beekman, Laurens Fortgens, Derk de Graaf, and Sander Vocke. They designed the first MEMS instruments, crafted the vacuum chamber, and made the control electronics. The instrument mask designs were simplified by Auke Been, Geert Folkertsma and Hylco de Boer before the mask design could be finalized for the micronium. The control electronics and capacitive read-out circuitry was improved by Hylco de Boer and Jasper Keuning. Esken Meutstege investigated tuning the notes electrostatically and electrothermally. Our lab technicians, Thijs Bolhuis, Marcel Kolster, Remco Sanders, Martin Siekman, and Henk van Wolferen, have proven themselves invaluable. Meint de Boer, Joël Geerlings, Kechun 'Kees' Ma, and Léon Woldering helped with the cleanroom fabrication.

Gerald Hoekstra created a movie of the 'В2 project', with which the presentation at MME 2010 started. Arvid Jense, from ArtEZ in Enschede, made the first composition for the demonstration of the micronium at $M M E 2010$, played live by a computer using a MIDI interface. The demonstration at the opening of the Nanolab featured pianist Daria Fedorchenko, also from ArtEZ, playing the instrument live. Hans Groenland filmed the performance at $M M E 2010$, the result of which was posted at Youtube and Vimeo and has travelled the internet. 


\section{Chapter 7}

\section{Conclusion and future outlook}

The most advanced scanner for probe-based data storage used today was developed by IBM, and is based on electrodynamic actuation. Next to its high speed, it has several disadvantages. Its energy consumption is relatively large and fabrication is complicated by the need for permanent magnets. A good alternative might be found in electrostatic comb-drive actuation, which targets these disadvantages. Although electrostatic stepper motors, notably the dipole surface drive and the shuffle drive, offer potentially very high forces at low voltages, their use is complicated, among others, by a relatively complex fabrication process and control circuit. Electrostatic comb-drive scanners are relative straightforward to fabricate, but the areal efficiency is generally low. A reported design using piezoelectric actuation with mechanical amplification is relatively young, and more research is required for a good judgement.

It is relatively straightforward to replace the electrodynamic actuator by an electrostatic comb-drive actuator in the mass-balanced $x / y$-scanner used in the 'Millipede' project by IBM, because the scanner is already fabricated from singlecrystal silicon using deep reactive-ion etching. Compared to the assembly of the electrodynamic scanner, the assembly of the scanner with an electrostatic combdrive is less complicated, because no coils or permanent magnets need be glued to the silicon device. However, electrical insulation between translator and stator of the electrostatic comb-drive requires break-out pieces that are hard to remove without damaging the scanner. To obtain an equal actuator force at $x=0$ at a maximum voltage of $150 \mathrm{~V}$, the electrostatic comb-drive must be designed larger than the electrodynamic actuator, leading to a decreased areal efficiency. However, the thickness of the electrostatic comb drive is less than the electrodynamic actuator. Combined, the electrostatic comb-drive scanner is more volume efficient than the electrodynamic scanner.

By using gap-narrowing comb-drive finger shapes instead of straight fingers, it is possible to obtain larger forces at equal maximum voltage and equal fabrication design limits. Tapering the fingers for the mass-balanced $x / y$-scanner doubles the obtainable equilibrium displacement. A twice as large shock-resistance compared 
to tapered fingers was obtained using the more aggressive gap-narrowing 'stepped' finger shape. However, the stepped shape results in a local maximum in the available force curve, which, if the maximum is too high, indicates unstable equilibrium displacement regions within the total displacement range. The optimal finger shape for maximum shock-resistance is a knife-like finger shape, that we named the 'optim' finger shape. The 'optim' shape results in a constant available force over the operating range. The asymmetric 'optim' shape has one straight edge, while the other edge is described by an analytical equation, and depends, among others, on the required available force, the spring stiffness, and the maximum voltage.

To study the energy consumption of different actuator types, three hypothetical scanner designs are discussed, one with an electrostatic comb-drive, another with an electromagnetic comb-drive, and the third with an electrodynamic actuator. The designs are such that the available force at $x=0$ is equal. Although the available-force curves of the comb-drive actuators are different from the electrodynamic actuator available force curve, the seek performance is roughly the same. There is however a large difference in the energy cost. The electrostatic comb-drive scanner is the most energy efficient; its energy cost for standing still or moving at $\mathrm{read} /$ write speed is 13 times less than the energy cost of the electrodynamic actuator and the electromagnetic comb-drive. Seek operations cost 3.4 and 5.8 times more energy on average for the electromagnetic comb-drive scanner and the electrodynamic scanner, respectively, than for the electrostatic comb-drive scanner. The energy cost is dominated by the dissipated energy in the leakage resistance of the electrostatic comb-drive and the series resistance of the coils in the magnetic scanners. Therefore, a good figure-of-merit for energy consumption is the dissipated energy at maximum voltage or current, $V_{\max }^{2} R_{\mathrm{p}}^{-1}$ for the electrostatic comb-drive, and $i_{\max }^{2} R_{\text {coil }}$ for the magnetic scanners (note that a lower figure-of-merit is better). The comparison does not take the efficiency of the driver circuitry into account. For equal energy consumption, the high-voltage driver circuitry for the electrostatic comb-drive can be 13 times less efficient than the current drivers for the electromagnetic comb-drive and the electrodynamic actuator. It is unlikely that the difference will be this large.

Electrostatic comb-drive actuators are a good alternative to electrodynamic actuation for probe-based data storage, offering better energy and volume efficiency combined with a higher shock resistance over the complete operating range. We therefore advise that future small form factor probe storage systems should be equipped with electrostatic comb-drive actuators with tailored finger shapes.

It is possible to fabricate a musical instrument using MEMS technology, consisting of resonators at audible frequencies, one for each note similar to a xylophone. The instrument has been named the 'micronium'. The micronium is placed in an ambient pressure of 1 mbar for a note duration around $1 \mathrm{~s}$. The micronium is outof-tune; fabrication variations result in audibly different resonance frequencies for resonators with identical dimensions. By carefully selecting resonators closest to the piano note frequencies, the micronium contains 16 notes that are agreeably in-tune on a range of almost 2 octaves. Because of the use of tapered comb-drive 
fingers, it is possible to tune the instrument by applying a constant offset voltage after the resonator has been excited. However, the maximum tuning ratio decreases with increasing resonator frequency and is smaller than one semitone. Further research is needed to asses the viability of other tuning methods. Creating a MEMS instrument is a very interesting and fun way to learn about MEMS. Future smaller musical MEMS projects are possible with an emphasis on generating original sounds instead of creating a whole instrument, using the acquired knowledge about MEMS instruments. The micronium project has received much media attention and has been quite successful in exposing MEMS technology to a broad, although probably mostly technically oriented, audience.

The presented work on comb-drive finger shapes is applicable to, besides probe data storage, nanopositioning in general, for example precision actuation in electron microscopes. A tailored finger shape can also improve the capacitive read-out signal in sensors like accelerometers. Whenever a comb drive is used, it is generally very rewarding to study tailored finger shapes instead of the standard straight shape.

There will not be a commercial probe data storage system in the near future. But, when further scaling of existing storage technologies is no longer possible or becomes too expensive, there may well be a revived interest in probe data storage systems at atomic resolution. Future work on actuators could focus on what the resolution limits are. Considering noise, what positioning accuracy can be obtained and maintained? What are the requirements for probe-based data storage at the atomic scale? For such requirements, it might be impossible to use a single positioning system for an array of many probes, due to thermal expansion of the probe array or medium platform. The possibility of multiple positioning systems for a small number of probes should be investigated. Electrostatic comb-drive actuators are an attractive option for a 'multi-scanner' system. In contrast to electromagnetic or piezoelectric actuators, electrostatic actuators scale favourably to smaller dimensions. Moreover, fabricating many comb-drives is as complex as fabricating one comb-drive, because the straightforward fabrication process does not require assembly of parts afterwards (no gluing of permanent magnets, coils, or piezoelectric material). 



\section{Appendices}





\section{Appendix A}

\section{Optimal charging of a capacitor}

In this appendix we will derive the energy optimal method of charging a capacitor without and with series and parallel resistors within a certain time $T$.

\section{A.1 Without leakage resistor}

To charge an ideal capacitance without parallel leakage resistor $R_{\mathrm{p}}$ through a series resistor $R_{\mathrm{s}}$, intuitively the optimal method is charging with a constant current. This can be proven as follows.

After transferring the required charge $Q=\int_{0}^{T} i \mathrm{~d} t$ to the capacitor, the total dissipated energy equals $E=R_{\mathrm{s}} \int_{0}^{T} i^{2} \mathrm{~d} t$. The optimal charge method minimizes the integral of the square of the current (the dissipated energy), for a fixed integral of the current (the charge).

The solution to this minimization problem can be found at home. Consider a basin filled with water. If we ignore the depth of the basin, and only consider the height and width $w$, we can define the water height $h(x)$, which can have a different value at each position $x$. The total amount of water in the basin equals $A=\int_{0}^{w} h \mathrm{~d} x$. The gravitational potential energy of a column of water of width $\Delta x$ equals $h \Delta x \rho g \frac{h}{2}$. The total potential energy equals $P=\frac{\rho g}{2} \int_{0}^{w} h^{2} \mathrm{~d} x$. Note that minimizing $P$ with constraint $A$ is equivalent to the optimal charging problem of a capacitor. The solutions are therefore the same: the water level $h(x)$ is constant in the basin; the optimal charge current $i(t)$ is a constant current.

\section{A.2 With leakage resistor}

The following calculation focusses on obtaining the optimal curve for the voltage across the capacitance $V$, from which the charge current can be inferred. 
The dissipation power equals the sum of the dissipation in $R_{\mathrm{s}}$ from the capacitor current $C \frac{\mathrm{d} V}{\mathrm{~d} t}$, and the dissipation in $R_{\mathrm{p}}$ and $R_{\mathrm{s}}$ by the current through $R_{\mathrm{p}}$,

$$
p_{\text {diss }}=R_{\mathrm{s}} C^{2}\left(\frac{\mathrm{d} V}{\mathrm{~d} t}\right)^{2}+\frac{V^{2}}{R_{\mathrm{p}}^{2}}\left(R_{\mathrm{s}}+R_{\mathrm{p}}\right) .
$$

Defining

$$
a=\sqrt{\frac{1}{R_{\mathrm{s}} C^{2}} \frac{R_{\mathrm{s}}+R_{\mathrm{p}}}{R_{\mathrm{p}}^{2}}} \approx \sqrt{\frac{1}{R_{\mathrm{p}} R_{\mathrm{s}} C^{2}}},
$$

we obtain the minimization problem

$$
\min _{V(t)} E_{\mathrm{diss}}[V(t)] ; \quad E_{\mathrm{diss}}=R_{\mathrm{s}} C^{2} \int_{0}^{T}\left(\frac{\mathrm{d} V}{\mathrm{~d} t}\right)^{2}+a^{2} V^{2} \mathrm{~d} t,
$$

with boundary conditions

$$
\begin{gathered}
V(t=0)=V_{1}, \\
V(t=T)=V_{2},
\end{gathered}
$$

where the capacitor is pre-charged at $V_{1}$ and $T$ is the time at which we want the capacitor to be charged at $V_{2}$.

Using the Euler-Lagrange equation (van Kan et al., 2005, chap. 5), minimization problem (A.1) with boundary conditions (A.2) and (A.3) is equivalent with the differential equation

$$
\frac{\mathrm{d}^{2} V}{\mathrm{~d} t^{2}}-a^{2} V=0
$$

Note that for infinite $R_{\mathrm{p}}, a=0$ and the solution for $V$ becomes a linear curve, consistent with $₫$ A.1. The solution for $V$ is

$$
V=D e^{a t}+F e^{-a t}
$$

Together with the boundary conditions, we obtain the values of $D$ and $F$,

$$
\begin{aligned}
D+F & =V_{1}, \\
D & =\frac{V_{2}-V_{1} e^{-a t}}{e^{a t}-e^{-a t}} .
\end{aligned}
$$

The optimal charge current equals

$$
i=C \frac{\mathrm{d} V}{\mathrm{~d} t}+\frac{V}{R_{\mathrm{p}}}=\sqrt{\frac{1}{R_{\mathrm{p}} R_{\mathrm{s}}}}\left(D e^{a t}-F e^{-a t}\right)+\frac{V}{R_{\mathrm{p}}} .
$$




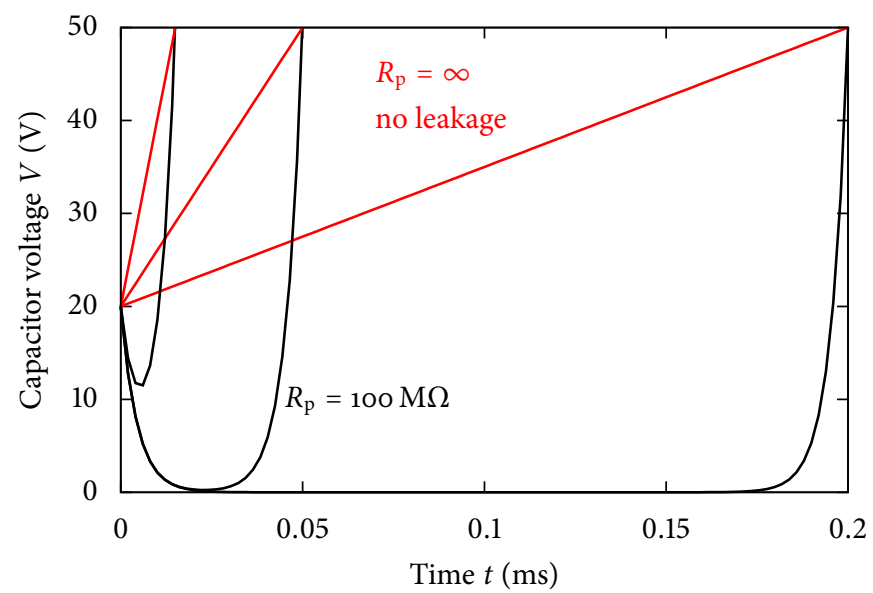

FIGURE A.1 - Optimal charge voltage waveform, minimizing the energy cost. Charge from $V_{1}=20 \mathrm{~V}$ to $V_{2}=50 \mathrm{~V}$ in a certain time $T=\{0.2,0.05,0.015\} \mathrm{ms}$. $C=45 \mathrm{pF}$ and $R_{s}=100 \Omega$.

Because $R_{\mathrm{s}} \ll R_{\mathrm{p}}$, the optimal supply voltage is approximately equal to voltage $V$ across the capacitor.

Figure A.1 shows the optimal voltage curves for different charge times $T$, and for two values of $R_{\mathrm{p}}$. It can be seen that, except for charge times below $50 \mu \mathrm{s}$, it is energy efficient to first discharge the capacitor, before charging it up to the desired voltage. This also means that an empty capacitor can be charged quite fast and energy efficient at the same time. When $V_{1}$ is zero, the solution for $V$ simplifies to

$$
V=V_{2} \frac{e^{a t}-e^{-a t}}{e^{a T}-e^{-a T}}
$$

and the dissipated energy as a function of charge time $T$ equals

$$
E_{\text {diss }}=V_{2}^{2} C \sqrt{\frac{R_{\mathrm{s}}}{R_{\mathrm{p}}}} \frac{e^{2 a T}+1}{e^{2 a T}-1} .
$$

The dissipated energy as a function of charge time $T$ is shown in Figure A.2. Charging from o $\mathrm{V}$ for a relatively long charge time $T$, the dissipated energy equals approximately $C V_{2}^{2} \sqrt{\frac{R_{\mathrm{s}}}{R_{\mathrm{p}}}}$, which in general is very small compared to $\frac{1}{2} C V_{2}^{2}$. We conclude that the energy cost of charging a discharged capacitor to $V_{2}$ requires just the stored energy $\frac{1}{2} C V_{2}^{2}$, because the dissipated energy is very small even when charging within $0.1 \mathrm{~ms}$.

Note that the square of the derivative of $V$ is added to the dissipated energy in (A.1). The minimization problem tries to minimize the energy cost also during 


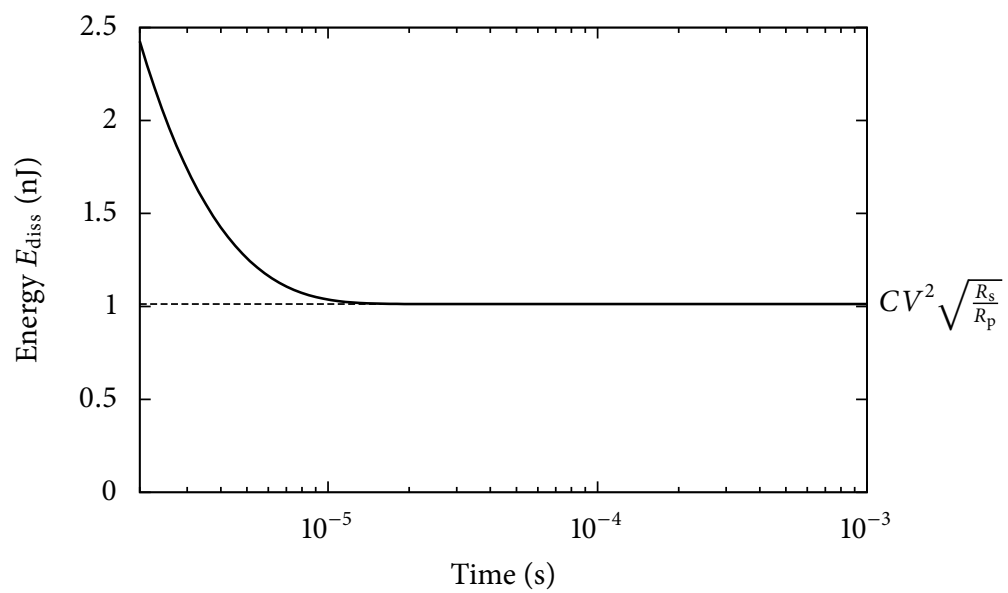

FIGURE A.2 - Optimal charge energy dissipated as function of charge time T. Charge to $150 \mathrm{~V}, C=45 \mathrm{pF}$ and $R_{s}=100 \Omega$. Compare with the stored energy $\frac{1}{2} C V^{2}=506 \mathrm{~nJ}$.

discharging, meaning that discharging adds to the total energy cost. When the solution for $V$ has a negative derivative, the solution is incorrect, because a better option may be to discharge the capacitor even more before starting to charge it again. Nevertheless, the shown solution is correct for charging from zero volt and indicates that for pre-charged capacitors, already for charge times less than $10 \mathrm{~ns}$ it is energy efficient to discharge the capacitor first. So, regardless of the initial voltage of the capacitor, when the capacitor should be charged up to $V_{2}$ in $0.5 \mathrm{~ms}$, say, the required energy equals $\frac{1}{2} C V_{2}^{2}$ to a very good approximation. 


\section{Appendix B}

\section{Seek time for electrodynamic actuator}

The given solution by Hong and Brandt (2002) for the seek time for the electrodynamic actuator requires calculation of a turnaround time $t_{\text {turnaround }}$ when either $v_{0}$ or $v_{1}$ is negative. A table is provided with information on when, and how many times $t_{\text {turnaround }}$ must be added to the seek time. However, only an approximate equation for $t_{\text {turnaround }}$ is given. Moreover, the equation by Hong and Brandt (2002) for the half-way point, $y_{\mathrm{m}}$, is not valid when $\left|v_{0}\right| \neq\left|v_{1}\right|$. Below, a more general solution is given for $t_{\text {seek,Ltz }}=t_{\text {accel }}+t_{\text {decel }}$, which is valid for all $v_{0}$ and $v_{1}$ when $x_{0} \leq x_{1}$.

To obtain our solution for the seek time we require use of the trigonometric identity

$$
p \sin \omega t+q \cos \omega t=\sqrt{p^{2}+q^{2}} \sin (\omega t+\phi),
$$

where

$$
\phi= \begin{cases}\arcsin \left(\frac{q}{\sqrt{p^{2}+q^{2}}}\right) & \text { if } p \geq 0, \\ -\pi-\arcsin \left(\frac{q}{\sqrt{p^{2}+q^{2}}}\right) & \text { if } p<0,\end{cases}
$$

or equivalently

$$
\phi=\arctan \left(\frac{q}{p}\right)+ \begin{cases}0 & \text { if } p \geq 0, \\ \pi & \text { if } p<0 .\end{cases}
$$

This identity is used to rewrite equations of the form

$$
p \sin \omega t+q \cos \omega t=r
$$

as

$$
\frac{r}{\sqrt{p^{2}+q^{2}}}=\sin (\omega t+\phi),
$$

which is solved for $t$ by taking the arcsine of both sides. 
Note that equation 18 from Hong and Brandt (2002) is of the same form as (A.3) above. Because $v_{0}$ can be negative, the solution for acceleration time $t_{\text {accel }}$ has to be split, resulting in

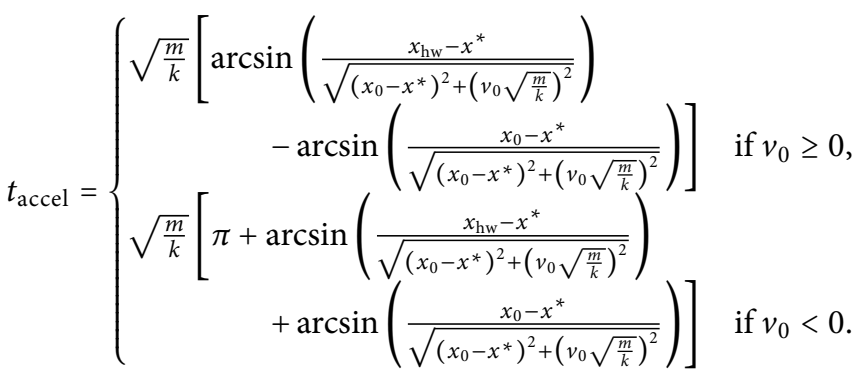

Note that the maximum equilibrium displacement

$$
x^{*}=\frac{m a_{0}}{k},
$$

see (5.5). The solution for the deceleration time $t_{\text {decel }}$ is found by multiplying equation 22 from Hong and Brandt (2002) by -1 , leading to

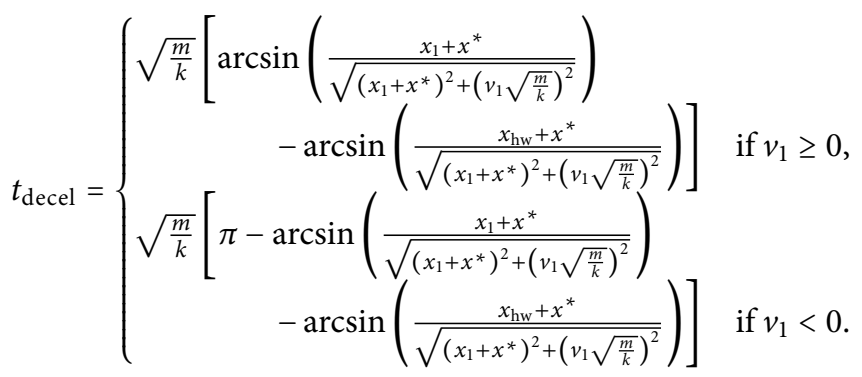

Note that the result is identical to the result of Hong and Brandt (2002) when $v_{0} \geq 0$ and $v_{1} \geq 0$, when (5.9) is used for $x_{\mathrm{hw}}$.

A more compact notation is obtained when (A.2) is used instead of (A.1):

$$
\begin{aligned}
& t_{\mathrm{accel}}=\sqrt{\frac{m}{k}}\left[\arcsin \left(\frac{x_{\mathrm{hw}}-x^{*}}{\sqrt{\left(x_{0}-x^{*}\right)^{2}+\left(v_{0} \sqrt{\frac{m}{k}}\right)^{2}}}\right)-\arctan \left(\frac{x_{0}-x^{*}}{v_{0} \sqrt{\frac{m}{k}}}\right)+\pi_{0}\right], \\
& t_{\text {decel }}=\sqrt{\frac{m}{k}}\left[\arcsin \left(\frac{-x_{\mathrm{hw}}-x^{*}}{\sqrt{\left(x_{1}+x^{*}\right)^{2}+\left(v_{1} \sqrt{\frac{m}{k}}\right)^{2}}}\right)+\arctan \left(\frac{x_{1}+x^{*}}{v_{1} \sqrt{\frac{m}{k}}}\right)+\pi_{1}\right],
\end{aligned}
$$

where

$$
\pi_{i}= \begin{cases}0 & \text { if } v_{i} \geq 0 \\ \pi & \text { if } v_{i}<0\end{cases}
$$


The implementation of these calculations should be validated with care. Especially when mirror transformation $\mathcal{M}$ (defined in (5.1)) is applied, $v_{0}$ or $v_{1}$ may be assigned the value ' -0 '. For correct calculation of $t_{\text {accel }}$ and $t_{\text {decel }}$,

$$
\pi_{i}+\arctan \left(\frac{1}{v_{i}}\right)=\frac{\pi}{2} \text { if } v_{i} \in\{-0,0,+0\} .
$$

For example, Matlab and common $\mathrm{C}$ math libraries distinguish between 0 and -0 : $\arctan \left(\frac{1}{0}\right)=\frac{1}{2} \pi$ and $\arctan \left(\frac{1}{-0}\right)=-\frac{1}{2} \pi$. However, the comparison ' $-0=0$ ' is true and ' $-0<0$ ' is false in Matlab, and therefore, if not carefully implemented, $\pi_{i}=0$ for $v_{i}=-0$, violating (A.4). The calculation result for $t_{\text {accel }}$ will then be negative and incorrect for $\left(x_{0}, x_{1}, v_{0}, v_{1}\right)=\left(0, x_{1},-0, v_{1}\right)$. A simple fix for this problem is to make a special implementation for when $v \in\{-0,0,+0\}$. 



\section{Appendix C}

\section{Process Flow}

The 'optim' comb drives (Chapter 4) and the micronium (Chapter 6) are fabricated using the same cleanroom fabrication process. The fabrication process consists of five steps. Starting with a silicon-on-insulator (SOI) wafer, first (1) a photoresist layer is patterned by lithography, then (2) the pattern is transferred into the SOI wafer's device layer by vertically etching down to the silicon oxide using deep reactive-ion etching (DRIE), subsequently (3) the wafer is diced, but not completely through, then (4) the exposed silicon oxide is etched using HF vapour-phase etching (VHF etching) to release the moving structures, and finally (5) the wafer is broken along the dice lines to obtain $7 \mathrm{~mm} \times 7 \mathrm{~mm}$ individual chips. The only difference between the 'optim' comb drives and the micronium is the oxide layer thickness of $1 \mu \mathrm{m}$ and $3 \mu \mathrm{m}$, respectively. The oxide thickness is increased for the micronium to reduce sticking problems caused by the large moving masses.

The design rules of the used fabrication process are listed in Table C.1. Moving structures should be perforated with holes, such that the HF vapour can reach and etch the silicon oxide under the moving structure.

TABLE C.1 - The design rules of the used fabrication process.

\begin{tabular}{lr}
\hline Quantity & Value \\
\hline Minimum beam width & $3 \mu \mathrm{m}$ \\
Minimum gap width & $3 \mu \mathrm{m}$ \\
Minimum fixed anchor size & $50 \mu \mathrm{m} \times 50 \mu \mathrm{m}$ \\
Moving structure perforation hole & $5 \mu \mathrm{m} \times 5 \mu \mathrm{m}$ \\
\multicolumn{1}{c}{$\quad$ perforation grid width } & $3 \mu \mathrm{m}$ \\
Expected silicon oxide underetch & $20 \mu \mathrm{m}$ \\
\hline
\end{tabular}


TABLE C.2 - The fabrication process details.

\begin{tabular}{|c|c|c|c|}
\hline & Process & & Note \\
\hline 1 & $\begin{array}{l}\text { Substrate selection } \\
\text { SOI }\end{array}$ & $\begin{array}{l}\text { Supplier: Okmetic } \\
\text { Bonded SOI wafer } 380-1.0-50 \\
\text { Growth method: CZ } \\
\text { Crystal orientation: }(100) \pm 0.2^{\circ} \\
\text { Diameter: } 100.0 \mathrm{~mm} \pm 0.2 \mathrm{~mm} \\
\text { Device layer } \\
\text { Thickness: } 50.0 \mu \mathrm{m} \pm 0.5 \mu \mathrm{m} \\
\text { Resistivity: } 0.01-0.02 \Omega \mathrm{cm} \\
\text { Type / Dopant: } \mathrm{P}+\text { / Boron } \\
\text { Oxide layer } \\
\text { Thickness: } 3.0 \mu \mathrm{m} \pm 0.1 \mu \mathrm{m} \\
\text { Handle wafer } \\
\text { Thickness: } 380 \mu \mathrm{m} \pm 5 \mu \mathrm{m} \\
\text { Resistivity: } 0.01-0.02 \Omega \mathrm{cm} \\
\text { Type / Dopant: } \mathrm{P}+/ \text { Boron }\end{array}$ & \\
\hline 2 & Standard Cleaning & $\begin{array}{l}\mathrm{HNO}_{3}(100 \%) \text { Selectipur: MERCK } \\
\mathrm{HNO}_{3}(69 \%) \text { VLSI: } \mathrm{MERCK}^{\circ} \\
\circ \text { Beaker 1: fumic } \mathrm{HNO}_{3}(100 \%) \text {, } \\
5 \text { min } \\
\circ \text { Beaker 2: fumic } \mathrm{HNO}_{3}(100 \%) \text {, } \\
5 \text { min } \\
\circ \text { Quick Dump Rinse }<0.1 \mu \mathrm{S} \\
\circ \text { Beaker 3: boiling }\left(95^{\circ} \mathrm{C}\right) \mathrm{HNO}_{3} \\
\text { (69\%), 10 min } \\
\circ \text { Quick Dump Rinse < } 0.1 \mu \mathrm{S} \\
\circ \text { Spin drying }\end{array}$ & \\
\hline 3 & $\begin{array}{l}\text { Lithography } \\
\text { Priming (liquid) }\end{array}$ & $\begin{array}{l}\text { SUSS MicroTec Spinner (Delta } 20) \\
\text { Hotplate } 120^{\circ} \mathrm{C} \\
\text { HexaMethylDiSilazane (HMDS) } \\
\text { - Dehydration bake: } 120^{\circ} \mathrm{C}, 5 \mathrm{~min} \\
\text { - Spin program: } 4 \text { (400o } \mathrm{rpm}, 20 \mathrm{~min})\end{array}$ & \\
\hline 4 & $\begin{array}{l}\text { Lithography } \\
\text { Coating }\end{array}$ & $\begin{array}{l}\text { SUSS MicroTec Spinner (Delta 20) } \\
\text { Hotplate } 95^{\circ} \mathrm{C} \\
\text { Photoresist: Olin } 907-17 \\
\text { - Spin Program: } 4 \text { (400o rpm, } 20 \mathrm{~min}) \\
\text { - Prebake: } 95^{\circ} \mathrm{C}, 90 \mathrm{~s}\end{array}$ & \\
\hline 5 & $\begin{array}{l}\text { Lithography } \\
\text { Exposure }\end{array}$ & $\begin{array}{l}\text { Electronic Vision Group } 620 \text { Mask } \\
\text { Aligner } \\
\text { ○ Hg-lamp: } 12 \mathrm{~mW} \mathrm{~cm}{ }^{-2} \\
\text { - Exposure Time: } 3.5 \mathrm{~s} \\
\text { - Vacuum contact mode }\end{array}$ & \\
\hline
\end{tabular}


TABle C.2 - Continued...

\begin{tabular}{|c|c|c|c|c|c|}
\hline \multicolumn{5}{|c|}{ Process } & \multirow[t]{2}{*}{ Note } \\
\hline 6 & $\begin{array}{l}\text { Lithography } \\
\text { Development }\end{array}$ & \multicolumn{3}{|c|}{$\begin{array}{l}\text { Hotplate } 120^{\circ} \mathrm{C} \\
\text { Developer: OPD4262 } \\
\text { O After Exposure Bake: } 120^{\circ} \mathrm{C}, 60 \mathrm{~s} \\
\text { - Time: } 30 \mathrm{~s} \text { in beaker } 1 \\
\text { - Time: } 15-30 \mathrm{~s} \text { in beaker } 2 \\
\text { - Quick Dump Rinse }<0.1 \mu \mathrm{S} \\
\text { - Spin drying }\end{array}$} & \\
\hline \multirow[t]{3}{*}{7} & $\begin{array}{l}\text { Plasma etching of } \\
\text { Si } \\
\text { B-ARCE-TWIN }\end{array}$ & \multicolumn{3}{|c|}{$\begin{array}{l}\text { Adixen SE } \\
\text { Aspect Ratio Controled Etching } \\
\text { (reducing-RIE lag) } \\
\text { Depth: } 50 \mu \mathrm{m} \\
\text { Time: } \sim 24 \mathrm{~min} \\
\end{array}$} & $\begin{array}{l}\text { Etch time can differ } \\
\text { depending on loading } \\
\text { differences between } \\
\text { mask designs. }\end{array}$ \\
\hline & & Parameters & Etch & Deposition & \\
\hline & & $\begin{array}{l}\text { Gas } \\
\text { Flow } \\
\text { Time } \\
\text { Priority } \\
\text { APC } \\
\text { ICP } \\
\text { CCP (LF) } \\
\text { Pulsed (LF) ms } \\
\text { He } \\
\text { SH } \\
\text { Electrode temp. }\end{array}$ & $\begin{array}{l}\mathrm{SF}_{6} \\
400 \mathrm{sccm} \\
5 \mathrm{~s} \\
2 \\
100 \% \\
1500 \mathrm{~W} \\
100 \mathrm{~W} \\
10 \mathrm{~ms} \text { on } \\
90 \mathrm{~ms} \text { off } \\
10 \mathrm{mbar} \\
200 \mathrm{~mm} \\
0^{\circ} \mathrm{C}\end{array}$ & $\begin{array}{l}\mathrm{C}_{4} \mathrm{~F}_{8} \\
280 \mathrm{sccm} \\
2 \mathrm{~s} \\
1 \\
100 \% \\
1500 \mathrm{~W} \\
100 \mathrm{~W} \\
10 \mathrm{~ms} \text { on } \\
90 \mathrm{~ms} \mathrm{off} \\
10 \mathrm{mbar} \\
200 \mathrm{~mm} \\
0{ }^{\circ} \mathrm{C}\end{array}$ & \\
\hline 8 & $\begin{array}{l}\text { Stripping of PR } \\
\text { by oxygen plasma }\end{array}$ & \multicolumn{4}{|c|}{$\begin{array}{l}\text { Tepla 30oE } \\
\text { Barrel Etcher }(2.45 \mathrm{GHz}) \\
\text { Multipurpose sytem } \\
\text { O O2 flow: } 200 \mathrm{sccm}(50 \%) \\
\text { O Power: } 500 \mathrm{~W} \\
\text { ○ Pressure: } 1.2 \text { mbar } \\
\text { - Time: } 10 \text { min for } 1-3 \text { wafers, } \\
20 \text { min for 4-10 wafers }\end{array}$} \\
\hline 9 & $\begin{array}{l}\text { Stripping in } \mathrm{HNO}_{3} \\
\text { multipurpose }\end{array}$ & \multicolumn{4}{|c|}{$\begin{array}{l}\mathrm{HNO}_{3}(100 \%) \text { Selectipur: MERCK } \\
100453 \\
\circ \mathrm{HNO}_{3}(100 \%), 20 \mathrm{~min} \\
\circ \text { Quick Dump Rinse < } 0.1 \mu \mathrm{S} \\
\circ \text { Spin drying }\end{array}$} \\
\hline
\end{tabular}


Table C.2 - Continued...

\begin{tabular}{|c|c|c|c|}
\hline & Process & & Note \\
\hline 10 & Dry Oxidation & $\begin{array}{l}\text { Furnace } \mathrm{B}_{3} \\
\circ \text { Program: DOX-80o } \\
\circ \text { Temperature: } 800{ }^{\circ} \mathrm{C} \\
\circ \text { Gas: } \mathrm{O}_{2} \\
\circ \text { Flow: } 41 \mathrm{~min}^{-1} \\
\circ \text { Time: } 30 \mathrm{~min}\end{array}$ & \\
\hline 11 & $\begin{array}{l}\text { Protect structures } \\
\text { for dicing }\end{array}$ & $\begin{array}{l}\text { SUSS MicroTec Spinner (Delta 20) } \\
\text { Hotplate } 95^{\circ} \mathrm{C} \\
\text { Photoresist: Olin } 908-35 \\
\text { - Spin Program: } 4 \text { (40oo rpm, } 20 \mathrm{~min}) \\
\circ \text { Bake: } 95^{\circ} \mathrm{C}, 60 \mathrm{~s}\end{array}$ & $\begin{array}{l}\text { Apply multiple times } \\
\text { until structures are } \\
\text { covered with a thick } \\
\text { resist layer. }\end{array}$ \\
\hline 12 & Dicing & $\begin{array}{l}\text { Disco DAD dicing saw } \\
\text { Nitto STW T10 dicing foil } \\
\text { Parameters dicing } \\
\text { Wafer work size: } 110 \mathrm{~mm} \\
\text { Max. feed speed: } 10 \mathrm{~mm} \mathrm{~s}^{-1} \\
\text { Blade details } \\
\text { Saw type: NBC-Z } 2050 \\
\text { Exposure: } 1.3 \mathrm{~mm} \\
\text { Width: } 50 \mu \mathrm{m} \\
\text { Spindle revolutions: } 30 \text { ooo rpm } \\
\text { Depth settings } \\
\text { Maximum cut depth: } 1.1 \mathrm{~mm} \\
\text { Foil thickness: } 80 \mu \mathrm{m} \\
\text { Min. blade height: } 50 \mu \mathrm{m} \\
\text { Remaining height: } 175 \mu \mathrm{m} \text { measured } \\
\text { from dice table } \\
\text { Dice pitch: } 7.2 \mathrm{~mm}\end{array}$ & $\begin{array}{l}\text { Note the dice guides } \\
\text { etched around the edge } \\
\text { of the wafer. Dice } 9 \\
\text { lines in X and } 9 \text { lines in } \\
\text { Y direction. Dice from } \\
\text { frontside of wafer. }\end{array}$ \\
\hline 13 & $\begin{array}{l}\text { Stripping in } \mathrm{HNO}_{3} \\
\text { multipurpose }\end{array}$ & $\begin{array}{l}\mathrm{HNO}_{3}(100 \%) \text { Selectipur: MERCK } \\
100453 \\
\circ \mathrm{HNO}_{3}(100 \%),>30 \text { min until clean } \\
\circ \text { Quick Dump Rinse < } 0.1 \mu \mathrm{S} \\
\circ \text { Spin drying }\end{array}$ & $\begin{array}{l}\text { It is paramount that } \\
\text { there is no photoresist } \\
\text { left in the perforation } \\
\text { holes. }\end{array}$ \\
\hline 14 & $\begin{array}{l}\mathrm{HF} \text { vapour-phase } \\
\text { etching of } \mathrm{SiO}_{2}\end{array}$ & $\begin{array}{l}\text { Idonius Vapor HF Tool } \\
\circ \text { Basin start temperature: } 20^{\circ} \mathrm{C} \\
\circ \text { Chuck temperature: } 35^{\circ} \mathrm{C} \\
\circ \text { Time: } 30 \text { min }\end{array}$ & $\begin{array}{l}\text { There are test squares } \\
\text { on the mask to deter- } \\
\text { mine the underetch, } \\
\text { which should be more } \\
\text { than } 7 \mu \mathrm{m} \text {. }\end{array}$ \\
\hline
\end{tabular}





\title{
Bibliography
}

\author{
Adams D N, 1979 \\ The Hitchiker's Guide to the Galaxy, \\ Pan Books
}

Agarwal M, Dutton D T, Theil J A, Bai Q, Par E, Hoen S, 2006

"Characterization of a dipole surface drive actuator with large travel and force"

J. Microelectromech. Syst. 15, pp. 1726-1734, doi:10.1109/JMEMS.2006.883886

Alfaro J F, Fedder G K, 2002

"Actuation for probe-based mass data storage"

In: 2002 International Conference on Modeling and Simulation of Microsystems - MSM 2002, pp. 202-205, San Juan, Puerto Rico

Bell D J, Lu T J, Fleck N A, Spearing S M, 2005

"MEMS actuators and sensors: Observations on their performance and selection for purpose"

J. Micromech. Microengineering 15, pp. 153-164, doi:10.1088/0960-1317/15/7/022

Bennewitz R, Crain J N, Kirakosian A, Lin J L, McChesney J L, Petrovykh D Y, Himpsel F J, 2002

"Atomic scale memory at a silicon surface"

Nanotechnol. 13, pp. 499-502, doi:10.1088/0957-4484/13/4/312

Binning G, Rohrer H, Gerber Ch, Weibel E, 1982

"Surface studies by scanning tunneling microscopy"

Phys. Rev. Lett. 49, pp. 57-61, doi:10.1103/PhysRevLett.49.57

Brouwer D M, Otten A, Engelen J B C, Krijnen B, Soemers H M J R, 31 May-4 Jun. 2010 "Long-range elastic guidance mechanisms for electrostatic comb-drive actuators" In: Proc. 1oth Int. Conf. of the European Society for Precision Engineering \& Nanotechnology (euspen 2010), pp. 47-50, Delft, the Netherlands

Bustillo J M, Howe R T, Muller R S, 1998

"Surface micromachining for microelectromechanical systems"

Proc. IEEE 86, pp. 1552-1573, doi:10.1109/5.704260

Cannara R J, Gotsmann B, Knoll A, Dürig U, 2008

"Thermo-mechanical probe storage at Mbps single-probe data rates and Tbit in ${ }^{-2}$ densities"

Nanotechnol. 19, p. 395305, doi:10.1088/0957-4484/19/39/395305

Carley L R, Bain J A, Fedder G K, Greve D W, Guillou D F, Lu M S C, Mukherjee T, Santhanam S, Abelmann L, Min S, 2000 "Single-chip computers with microelectromechanical systems-based magnetic memory" J. Appl. Phys. 87, pp. 668o-6685, doi:10.1063/1.372807

Carley L R, Ganger G, Guillou D F, Nagle D, 2001 "System design considerations for MEMS-actuated magnetic-probe-based mass storage" IEEE Trans. Magn. 37, pp. 657-662, doi:10.1109/20.917597 
Carr D W, Evoy S, Sekaric L, Craighead H G, Parpia J M, 1999

"Measurement of mechanical resonance and losses in nanometer scale silicon wires"

Appl. Phys. Lett. 75, pp. 920-922, doi:10.1063/1.124554

Chen C H, Yeh J A, Wang P J, 2006

"Electrical breakdown phenomena for devices with micron separations"

J. Micromech. Microeng. 16, pp. 1366-1373, doi:10.1088/0960-1317/16/7/034

Cheung P, Horowitz R, Rowe R T, 1996

"Design, fabrication, position sensing, and control of an electrostatically-driven polysilicon microactuator"

IEEE Trans. Magn. 32, pp. 122-128, doi:10.1109/20.477561

Cho C H, 2009

"Characterization of Young's modulus of silicon versus temperature using a "beam deflection" method with a four-point bending fixture"

Curr. Appl. Phys. 9, pp. 538-545, doi:10.1016/j.cap.2008.03.024

Choi J J, Park H, Kim K Y, Jeon J U, 2001 a

"Electromagnetic micro $\mathrm{x}-\mathrm{y}$ stage for probe-based data storage"

J. Semicond. Technol. Sci. 1, pp. 84-93

Choi J J, Park H, Kim K Y, Jeon J U, $2001 b$

"Electromagnetic micro $\mathrm{x}-\mathrm{y}$ stage with very thick $\mathrm{Cu}$ coil for probe-based mass data storage device"

In: Proc. SPIE, volume 4334, pp. 363-371, Newport Beach, CA, USA, doi:10.1117/12.436622

Comstock R H, Apr. 1981

"Charge control of piezoelectric actuators to reduce hysteresis effects"

U.S. Patent 4263 527, Apr., 1981

Cornell University Science News, 1997

"World's smallest silicon mechanical devices are made at Cornell"

URL http: //www . news. cornell.edu/releases/july97/guitar.ltb.html

Despont M, Drechsler U, Yu R, Pogge H B, Vettiger P, 2004

"Wafer-scale microdevice transfer/interconnect: Its application in an AFM-based data-storage system"

J. Microelectromech. Syst. 13, pp. 895-901, doi:10.1109/JMEMS.2004.835769

Eigler D M, Schweizer E K, 1990

"Positioning single atoms with a scanning tunnelling microscope"

Nature 344, pp. 524-526, doi:10.1038/344524a0

Eleftheriou E, Antonakopoulos T, Binnig G K, Cherubini G, Despont M, Dholakia A, Durig U, Lantz M A, Pozidis H, Rothuizen H E, Vettiger P, 2003

"Millipede-A MEMS-based scanning-probe data-storage system"

IEEE Trans. Magn. 39, pp. 938-945, doi:10.1109/TMAG.2003.808953

Faizul M S, Ono T, Esashi M, 21-25 Jun. 2009a

"A large displacement piezodriven silicon XY-microstage"

In: Int. Solid-State Sensors, Actuators and Microsystems Conf. (TRANSDUCERS 2009), pp. 24052408, Denver, CO, USA, doi:10.1109/SENSOR.2009.5285429

Faizul M S, Ono T, Esashi M, $2009 b$

"Modeling and experimental validation of the performance of a silicon XY-microstage driven by PZT actuators"

J. Micromech. Microeng. 19, p. o95004, doi:10.1088/0960-1317/19/9/095004 
Felten E, Sept. 2010

"The era of infinite storage"

Sci. Am. 303, p. 94

Feynman R P, 29 Dec. 1959

"There's plenty of room at the bottom"

URL http: //www.zyvex.com/nanotech/feynman.html

(accessed 15 Oct. 2010)

Fontana R E, Hetzler S R, 2009

"Millions of square inches (MSI) comparisons \& implications for magnetic and solid state storage class memories"

INSIC Annual Meeting Symposium

FreeFEM++ 3.8,

URL http: //www.freefem.org/ff++/index.htm

(accessed 17 Feb. 2010)

Gantz J F, Chute C, Manfrediz A, Minton S, Reinsel D, Schlichting W, Toncheva A, 2008

The Diverse \& Exploding Digital Universe: An Updated Forecast of Worldwide Information Growth Through 2011,

International Data Corporation

Gidon S, Lemonnier O, Rolland B, Bichet O, Dressler C, Samson Y, 2004

"Electrical probe storage using joule heating in phase change media"

Appl. Phys. Lett. 85, pp. 6392-6394, doi:10.1063/1.1834718

Grade J D, Jerman H, Kenny T W, 2003

"Design of large deflection electrostatic actuators"

J. Microelectromech. Syst. 12, pp. 335-343, doi:10.1109/JMEMS.2003.811750

Grade J D, Yasumura K Y, Jerman H, 2004

"Advanced, vibration-resistant, comb-drive actuators for use in a tunable laser source"

Sens. Actuators A 114, pp. 413-422, doi:10.1016/j.sna.2003.11.012

Griffin J L, Schlosser S W, Ganger G R, Nagle D F, 2000

"Modeling and performance of MEMS-based storage devices"

In: Proc. ACM SIGMETRICS 200o, volume 28, pp. 56-65, Santa Clara, CA, USA, doi:10.1145/345063.339354

Griffiths D J, 1999

Introduction to electrodynamics,

Prentice Hall, 3rd edition

Hartwell P G, Walmsley R G, Fasen D J, Hoen S, 24-27 Oct. 2004

"Integrated position sensing for control of XY actuator"

In: Proc. IEEE Sensors 2004, volume 3, pp. 1407-1410, Vienna, Austria, doi:10.1109/ICSENS.2004.1426448

Hidaka T, Maruyama T, Saitoh M, Mikoshiba N, Shimizu M, Shiosaki T, Wills L A, Hiskes R, Dicarolis S A, Amano J, 1996

"Formation and observation of $50 \mathrm{~nm}$ polarized domains in $\mathrm{pbzr}_{1-x} \mathrm{ti}_{x} \mathrm{O}_{3}$ thin film using scanning probe microscope"

Appl. Phys. Lett. 68, pp. 2358-2359, doi:10.1063/1.115857

Hoen S, Bai Q, Harley J A, Horsley D A, Matta F, Verhoeven T, Williams J, Williams K R, 2003

"A high-performance dipole surface drive for large travel and force"

In: 12th Int. Conf. on Solid-State Sensors, Actuators and Microsystems (TRANSDUCERS 2003),

volume 1, pp. 344-347, doi:10.1109/SENSOR.2003.1395506 
Holmes P J, Snell J E, 1966

"A vapour etching technique for the photolithography of silicon dioxide"

Microelectron. Reliab. 5, pp. 337-341, doi:10.1016/0026-2714(66)90162-4

Hong B, Brandt S A, 2002

"An analytical solution to a MEMS seek time model"

Technical Report UCSC-CRL-02-31, University of California, Santa Cruz

Hong D S, El-Gamal M N, 2003

"Low operating voltage and short settling time CMOS charge pump for MEMS applications"

ISCAS 'o3. Proceedings of the 2003 International Symposium on Circuits and Systems, 2003 5, pp. 281-284, doi:10.1109/ISCAS.2003.1206254

Huang X, Lee J I, Ramakrishnan N, Bedillion M, Chu P, 2010 a

"Nano-positioning of an electromagnetic scanner with a MEMS capacitive sensor"

Mechatronics 20, pp. 27-34, doi:10.1016/j.mechatronics.2009.06.005

Huang X, Lee J I, Ramakrishnan N, Bedillion M, Chu P, $2010 b$

"Nano-positioning of an electromagnetic scanner with a MEMS capacitive sensor"

Mechatronics 20, pp. 27-34, doi:10.1016/j.mechatronics.2009.06.005

Hubbard N B, Culpepper M L, Howell L L, 2006

"Actuators for micropositioners and nanopositioners"

Appl. Mech. Rev. 59, pp. 324-334, doi:10.1115/1.2345371

Jansen H V, De Boer M J, Unnikrishnan S, Louwerse M C, Elwenspoek M C, 2009

"Black silicon method X"

J. Micromech. Microeng. 19, p. 033001, doi:10.1088/0960-1317/19/3/033001

Jensen B D, Mutlu S, Miller S, Kurabayashi K, Allen J J, 2003

"Shaped comb fingers for tailored electromechanical restoring force"

J. Microelectromech. Syst. 12, pp. 373-383, doi:10.1109/JMEMS.2003.809948

Johnson W A, Warne L K, 1995

"Electrophysics of micromechanical comb actuators"

J. Microelectromech. Syst. 4, pp. 49-59, doi:10.1109/84.365370

van Kan J, Segal A, Vermolen F, 2005

Numerical methods in scientific computing,

VSSD, Delft, the Netherlands

Ker M D, Jiang H C, Chang C Y, 2001

"Design on the low-capacitance bond pad for high-frequency I/O circuits in CMOS technology" IEEE Trans. Electron Devices 48, pp. 2953-2956, doi:10.1109/16.974736

Khatib M G, Jun. 2009

MEMS-Based Storage Devices - Integration in Energy-Constrained Mobile Systems

Ph.D. thesis, University of Twente, Enschede, the Netherlands, doi:10.3990/1.9789036528474

Khatib M G, Miller E L, Hartel P H, Oct. 2008

"Workload-based Configuration of MEMS-Based Storage Devices for Mobile Systems"

In: Proceedings of the 8th ACM \& IEEE International Conference on Embedded Software (EMSOFT 'o8), pp. 41-50, ACM, Atlanta, USA, doi:10.1145/1450058.1450065

Kim C H, Jeong H M, Jeon J U, Kim Y K, 2003

"Silicon micro XY-stage with a large area shuttle and no-etching holes for SPM-based data storage" J. Microelectromech. Syst. 12, pp. 470-478, doi:10.1109/JMEMS.2003.809960 
Kotsopoulos A G, Antonakopoulos T A, 2010

"Nanopositioning using the spiral of Archimedes: The probe-based storage case"

Mechatronics 2o, pp. 273-28o, doi:10.1016/j.mechatronics.2009.12.004

Krijnen B, Hogervorst R P, Engelen J B C, van Dijk J W, Brouwer D M, Abelmann L, 26-29 Sept. 2010 "Single-mask thermal displacement sensor in MEMS"

In: Proc. Micromechanics and Microsystems Europe, Enschede, the Netherlands

Kuijpers A A, 2004

Micromachined capacitive long-range displacement sensor for nano-positioning of microactuator systems

Ph.D. thesis, University of Twente, Enschede, the Netherlands

Kuijpers A A, Krijnen G J M, Wiegerink R J, Lammerink T S J, Elwenspoek M, 2006

"A micromachined capacitive incremental position sensor: Part 2. Experimental assessment"

J. Micromech. Microeng. 16, pp. S125-S134, doi:10.1088/0960-1317/16/6/S19

Kwon H N, Lee J H, Takahashi K, Toshiyoshi H, 2006

"MicroXY stages with spider-leg actuators for two-dimensional optical scanning"

Sens. Actuators A 130-131, pp. 468-477, doi:10.1016/j.sna.2005.10.037

Laermer F, Schilp A, 1994

"Method of anisotropically etching silicon"

German Patent DE 4241045,1994

(U.S. Patent 5501893,1996$)$

Lantz M A, Binnig G K, Despont M, Drechsler U, 2005

"A micromechanical thermal displacement sensor with nanometre resolution"

Nanotechnol. 16, pp. 1089-1094, doi:10.1088/0957-4484/16/8/016

Lantz M A, Rothuizen H E, Drechsler U, Häberle W, Despont M, 2007

"A vibration resistant nanopositioner for mobile parallel-probe storage applications"

J. Microelectromech. Syst. 16, pp. 130-139, doi:10.1109/JMEMS.2006.886032

LaSalle J P, 1959

"Time optimal control systems"

Proc. Natl. Acad. Sci. U.S.A. 45, pp. 573-577

URL http://www.jstor.org/stable/90106

Lee J I, Huang X, Chu P B, 2009

"Nanoprecision MEMS capacitive sensor for linear and rotational positioning"

J. Microelectromech. Syst. 18, pp. 660-67o, doi:10.1109/JMEMS.2009.2016275

Legtenberg R, Groeneveld A W, Elwenspoek M C, 1996

"Comb-drive actuators for large displacements"

J. Micromech. Microeng. 6, pp. 320-329, doi:10.1088/0960-1317/6/3/004

Lu Y, Pang C K, Chen J, Zhu H, Yang J P, Mou J Q, Guo G X, Chen B M, Lee T H, 24-28 July 2005 "Design, fabrication and control of a micro X-Y stage with large ultra-thin film recoding media platform"

In: Proc. IEEE/ASME Int. Conf. Advanced Intelligent Mechatronics (AIM2005), pp. 19-24, Monterey, CA, USA, doi:10.1109/AIM.2005.1500959

Mahmood I A, Moheimani S O R, 2009

"Fast spiral-scan atomic force microscopy"

Nanotechnol. 20, p. 365503, doi:10.1088/0957-4484/20/36/365503

Mamin H J, Terris B D, Fan L S, Hoen S, Barrett R C, Rugar D, 1995

"High-density data storage using proximal probe techniques"

IBM J. Res. Dev. 39, pp. 681-699, doi:10.1147/rd.396.0681 
Mamin H J, Ried R P, Terris B D, Rugar D, 1999

"High-density data storage based on the atomic force microscope"

Proc. IEEE 87, pp. 1014-1027, doi:10.1109/5.763314

Min D K, Hong S, 2005

"Servo and tracking algorithm for a probe storage system"

IEEE Trans. Magn. 41, pp. 855-859, doi:10.1109/TMAG.2004.840347

Minne S C, Adams J D, Yaralioglu G, Manalis S R, Atalar A, Quate C F, 1998

"Centimeter scale atomic force microscope imaging and lithography"

Appl. Phys. Lett. 73, pp. 1742-1744, doi:10.1063/1.122263

Mohr J, Bley P, Strohrmann M, Wallrabe U, 1992

"Microactuators fabricated by the LIGA process"

J. Micromech. Microeng. 2, pp. 234-241, doi:10.1088/0960-1317/2/4/003

Morgan B, Ghodssi R, 2008

"Vertically-shaped tunable MEMS resonators"

J. Microelectromech. Syst. 17, pp. 85-92, doi:10.1109/JMEMS.2007.910251

Ohkubo T, Yanagisawa K, Kaneko R, Kishigami J, 1993

"Magnetic force microscopy for high-density point magnetic recording"

Electronics \& Communications in Japan, Part II: Electronics (English translation of Denshi Tsushin Gakkai Ronbunshi) 76, pp. 94-103

Okada H, Itoh T, Suga T, 2009

"Pressure dependence of resonant characteristics of lateral comb drive resonators in the freemolecule regime"

Appl. Phys. Express 2, p. 096501, doi:10.1143/APEX.2.096501

Pang C K, Lu Y, Li C, Chen J, Zhu H, Yang J, Mou J, Guo G, Chen B M, Lee T H, 2009

"Design, fabrication, sensor fusion, and control of a micro X-Y stage media platform for probebased storage systems"

Mechatronics 19, pp. 1158-1168, doi:10.1016/j.mechatronics.2009.03.005

Pantazi A, Lantz M A, Cherubini G, Pozidis H, Eleftheriou E, 2004

"A servomechanism for a micro-electromechanical-system-based scanning-probe data storage device"

Nanotechnol. 15, pp. 612-621, doi:10.1088/0957-4484/15/10/019

Pantazi A, Sebastian A, Cherubini G, Lantz M A, Pozidis H, Rothuizen H E, Eleftheriou E, 2007 "Control of MEMS-based scanning-probe data-storage devices"

IEEE Trans. Contr. Syst. T. 15, pp. 824-841, doi:10.1109/TCST.2006.890286

Pantazi A, Sebastian A, Antonakopoulos T A, Bächtold P, Bonaccio A R, Bonan J, Cherubini G, Despont M, DiPietro R A, Drechsler U, Dürig U, Gotsmann B, Häberle W, Hagleitner C, Hedrick J L, Jubin D, Knoll A, Lantz M A, Pentarakis J, Pozidis H, Pratt R C, Rothuizen H E, Stutz R, Varsamou M, Weismann D, Eleftheriou E, 2008

"Probe-based ultrahigh-density storage technology"

IBM J. Res. Dev. 52, pp. 493-511, doi:10.1147/rd.524.0493

Patrascu M, Sept. 2006

Characterization, modeling and control of the $\mu$ Walker - a micro actuator for data storage Ph.D. thesis, University of Twente, Enschede, the Netherlands

Patrascu M, Stramigioli S, de Boer M J, Krijnen G J M, Nov. 2007

"Nanometer range closed-loop control of a stepper micro-motor for data storage"

In: Proceedings of 2007 ASME International Mechanical Engineering Congress and Exposition, pp. 1-9, ASME, Seattle, WA, USA 
Planar Motion Analyzer 2.5, Polytec MSA40o, Polytec GmbH, URL http: //www . polytec.com

Pozidis H, Häberle W, Wiesmann D, Drechsler U, Despont M, Albrecht T R, Eleftheriou E, 2004 "Demonstration of thermomechanical recording at $641 \mathrm{Gbit} / \mathrm{in}^{2}$ "

IEEE Trans. Magn. 4o, pp. 2531-2536, doi:10.1109/TMAG.2004.830470

Remtema T, Lin L, 2001

"Active frequency tuning for micro resonators by localized thermal stressing effects"

Sens. Actuators A 9o, pp. 326-332, doi:10.1016/S0924-4247(01)00603-3

Rosa M A, Dimitrijev S, Harrison H B, 1998

"Enhanced electrostatic force generation capability of angled comb finger design used in electrostatic comb-drive actuators"

Electron. Lett. 34, pp. 1787-1788

Rothuizen H, Drechsler U, Genolet G, Haberle W, Lutwyche M, Stutz R, Widmer R, Vettiger P, 2000 "Fabrication of a micromachined magnetic $X / Y / Z$ scanner for parallel scanning probe applications"

Microelectron. Eng. 53s, pp. 509-512, doi:10.1016/S0167-9317(00)00366-X

Rothuizen H, Despont M, Drechsler U, Genolet G, Haberle W, Lutwyche M, Stutz R, Vettiger P, 2002 "Compact copper/epoxy-based electromagnetic scanner for scanning probe applications"

In: 15th Int. Conf. on Micro Electro Mechanical Systems (MEMS 2002), pp. 582-585, Las Vegas, NV, USA, doi:10.1109/MEMSYS.2002.984338

Saheb J F, Richard J F, Sawan M, Meingan R, Savaria Y, Oct. 2007

"System integration of high voltage electrostatic MEMS actuators"

Analog Integrated Circuits and Signal Processing 53, pp. 27-34, doi:10.1007/s10470-006-9020-x

Sarajlic E, Berenschot J W, Fujita H, Krijnen G J M, Elwenspoek M C, 30 Jan.-3 Feb. $2005 a$

"Bidirectional electrostatic linear shuffle motor with two degrees of freedom"

In: 18th Int. Conf. on Micro Electro Mechanical Systems (MEMS 2005), pp. 391-394, Miami, FL, USA, doi:10.1109/MEMSYS.2005.1453949

Sarajlic E, Berenschot J W, Tas N R, Fujita H, Krijnen G J M, Elwenspoek M C, 5-9 Jun. 2005b "High performance bidirectional electrostatic inchworm motor fabricated by trench isolation technology"

In: 13th Int. Conf. on Solid-State Sensors, Actuators and Microsystems, Digest of Technical Papers (TRANSDUCERS 2005), volume 1, pp. 53-56, Seoul, South Korea, doi:10.1109/SENSOR.2005.1496357

Sasaki M, Bono F, Hane K, 2008

"Large-displacement micro- $X Y$-stage with paired moving plates"

Jpn. J. Appl. Phys. 47, pp. 3226-3231, doi:10.1143/JJAP.47.3226

Schlosser S W, 2004

Using MEMS-based storage devices in computer systems

Ph.D. thesis, Carnegie Mellon University, Pittsburgh, PA, USA

Schonhardt S, Korvink J G, Mohr J, Hollenbach U, Wallrabe U, 2008

"Magnetic comb drive actuator"

In: 21st Int. Conf. on Micro Electro Mechanical Systems (MEMS 2008), pp. 479-482, Tucson, AZ, USA, doi:10.1109/MEMSYS.2008.4443697

Schonhardt S, Korvink J G, Mohr J, Hollenbach U, Wallrabe U, 2009

"Optimization of an electromagnetic comb drive actuator"

Sens. Actuators A 154, pp. 212-217, doi:10.1016/j.sna.2008.08.007 
Sebastian A, Pantazi A, Cherubini G, Eleftheriou E, Lantz M A, Pozidis H, 2005

"Nanopositioning for probe storage"

In: Proceedings of the American Control Conference, volume 6, pp. 4181-4186, Portland, OR, United States, doi:10.1109/ACC.2005.1470634

Sebastian A, Pantazi A, Cherubini G, Lantz M, Rothuizen H, Pozidis H, Eleftheriou E, 2006

"Towards faster data access: seek operations in MEMS-based storage devices"

In: 2006 IEEE International Conference on Control Applications, pp. 283-288, Munich, Germany, doi:10.1109/CACSD-CCA-ISIC.2006.4776660

Sebastian A, Pantazi A, Pozidis H, 2007

"Jitter investigation and performance evaluation of a small-scale probe storage device prototype" In: 5oth Annual IEEE Global Telecommunications Conference, GLOBECOM 2007, pp. 288-293,

Washington, DC, doi:10.1109/GLOCOM.2007.61

Sebastian A, Pantazi A, Reza Moheimani S O, Pozidis H, Eleftheriou E, 2008

"Achieving subnanometer precision in a MEMS-based storage device during self-servo write process"

IEEE Trans. Nanotechnol. 7, pp. 586-595, doi:10.1109/TNANO.2008.926441

Steele B, 2003

"New nanoguitar offers promise of applications in electronics, sensing"

URL http: //www. news . cornell.edu/Chronicle/03/11.20.03/new_nanoguitar.html

Syms R R A, 1998

"Electrothermal frequency tuning of folded and coupled vibrating micromechanical resonators"

J. Microelectromech. Syst. 7, pp. 164-171, doi:10.1109/84.679341

Tang W C, Nguyen T C H, Howe R T, 1989

"Laterally driven polysilicon resonant microstructures"

Sens. Actuators 2o, pp. 25-32, doi:10.1016/0250-6874(89)87098-2

Tas N, Wissink J, Sander L, Lammerink T, Elwenspoek M C, 1998

"Modeling, design and testing of the electrostatic shuffle motor"

Sens. Actuators A 70, pp. 171-178, doi:10.1016/S0924-4247(98)00129-0

Tilmans H A C, 1996

"Equivalent circuit representation of electromechanical transducers: I. lumped-parameter systems" J. Micromech. Microeng. 6, pp. 157-176, doi:10.1088/0960-1317/6/1/036

Erratum published in vol. 6, p. 359, 1996

UMECH MEMS Motion Analyzer, Umech Technologies,

URL http://www . umech.com

Vettiger P, Cross G, Despont M, Drechsler U, Dürig U, Gotsmann B, Häberle W, Lantz M A, Rothuizen H E, Stutz R, Binnig G K, 2002

"The "Millipede"-Nanotechnology entering data storage"

IEEE Trans. Nanotechnol. 1, pp. 39-54, doi:10.1109/TNANO.2002.1005425

Wiegerink R J, Floris A, Jaganatharaja R K, Izadi N, Lammerink T S J, Krijnen G J M, 28-31 Oct. 2007 "Biomimetic flow-sensor arrays based on the filiform hairs on the cerci of crickets" In: IEEE Sensors, pp. 1073-1076, Atlanta, GA, USA, doi:10.1109/ICSENS.2007.4388591

Woodson H H, Melcher J R, 1968

Electromechanical Dynamics, Part I,

Massachusetts Institute of Technology: MIT OpenCourseWare

URL http: //ocw.mit.edu

(accessed 17 Aug. 2010) 
Yang J P, Mou J Q, Chong N B, Lu Y, Zhu H, Jiang Q, Kim W G, Chen J, Guo G X, Ong E H, 2007 "Probe recording technology using novel MEMS devices"

Microsyst. Technol. 13, pp. 733-740, doi:10.1007/s00542-006-0267-z

Yao J J, MacDonald N C, 1995

"A micromachined, single-crystal silicon, tunable resonator"

J. Micromech. Microeng. 5, pp. 257-264, doi:10.1088/0960-1317/5/3/009

Yao J J, Arney S C, MacDonald N C, 1992

"Fabrication of high frequency two-dimensional nanoactuators for scanned probe devices"

J. Microelectromech. Syst. 1, pp. 14-22, doi:10.1109/84.128051

Ye W, Mukherjee S, MacDonald N C, 1998

"Optimal shape design of an electrostatic comb drive in microelectromechanical systems"

J. Microelectromech. Syst. 7, pp. 16-26, doi:10.1109/84.661380

Yntema D R, Haneveld J, Engelen J B C, Brookhuis R A, Sanders R G P, Wiegerink R J, Elwenspoek M, 24-28 Jan. 2010

"Listening to MEMS: An acoustic vibrometer"

In: Proc. IEEE MEMS 2010, pp. 663-666, Hong Kong, China, doi:10.1109/MEMSYS.2010.5442319

Young R W, 1939

"Terminology for logarithmic frequency units"

J. Acoust. Soc. Am. 11, pp. 134-139, doi:10.1121/1.1916017

Zhang D, Chang C, Ono T, Esashi M, 2003

"A piezodriven XY-microstage for multiprobe nanorecording"

Sens. Actuators A 108, pp. 230-233, doi:10.1016/S0924-4247(03)00373-X 



\section{Summary}

The era of infinite storage seems near. To reach it, data storage capabilities need to grow, and new storage technologies must be developed. This thesis studies one aspect of one of the emergent storage technologies: optimizing electrostatic combdrive actuation for a parallel probe-based data storage system.

Chapter 1 introduces the data storage problem: it is no longer possible to store all created information. To combat data loss, there is a demand for increased data storage capabilities. New storage technologies must be developed as current commercial technologies reach their fundamental limits. One promising technology is parallel probe-based data storage, using a nanometre-scale probe to write data on a moving platform (Chapter 1). The working principle is very similar to that of a record player applied in parallel on the nano scale. In order to access all bits on the storage medium, a nano-positioner, or scanner, is used to move the storage medium relative to the read-out probes.

Scanning the literature (Chapter 2) several nano-positioner designs for probe data storage are found. It is not clear which actuator type (electromagnetic, electrostatic, or piezoelectric) is most suited for probe data storage. Concessions must be made in the design of a scanner to satisfy all requirements. We replaced the electrodynamic actuators by comb-drives in the scanner prototype by IBM, and the result still seems to strike a good balance (Chapter 3 ). However, the areal efficiency is low, due to a relatively low electrostatic force. To increase the force, the combdrive finger profile is optimized for increased shock resistance, putting electrostatic comb-drives in better shape for probe data storage (Chapter 4).

The suitability of electromagnetic and electrostatic actuation is, among others, determined by their hunger for power (Chapter 5). Three (partly) hypothetical scanner designs using electrodynamic, electromagnetic and electrostatic comb-drive actuators are described. Their performance is approximately equal, however electrostatic comb-drive actuation requires an order of magnitude less energy. Equations are presented for further investigations into the performance and energy consumption of the different actuation types for different file-system use cases.

Chapter 6 muses on the possibility of making music with MEMS structures. We were successful and named our micro-instrument 'the micronium'. Due to fabrication inaccuracies, the instrument is out-of-tune and requires tuning. Besides teaching students about MEMS technology in a fun way, the micronium succeeded in presenting MEMS technology to a broad audience. 


\section{Samenvatting}

Het tijdperk van oneindige opslagcapaciteit nadert. Om dit te bereiken zullen data opslag mogelijkheden moeten doorgroeien, maar ook nieuwe opslag technieken moeten ontwikkeld worden. Dit proefschrift bestudeert een detail van één van de opkomende opslag technieken: het optimaliseren van elektrostatische comb drive actuatie in parallelle sonde-gebaseerde data opslag.

Niet alle gegenereerde informatie kan meer opgeslagen worden (hfst. 1). Er is daarom behoefte aan grotere data opslag mogelijkheden. Nieuwe opslag technieken moeten ontwikkeld worden, omdat huidige technieken hun natuurkundige limieten bereiken. Een veelbelovende technologie is parallelle sonde-gebaseerde data opslag: het gebruik van nanometer sondes voor het schrijven van gegevens op een bewegend platform (hfst. 1). Het werkingsprincipe is vergelijkbaar met dat van een platenspeler toegepast op de nanoschaal. Een 'scanner' wordt gebruikt om het opslagmedium ten opzichte van de uitlees sondes te bewegen.

In de literatuur (hfst. 2) staan enkele ontwerpen van scanners, specifiek voor sonde data-opslag. Verschillende soorten actuatoren worden gebruikt, en het is niet duidelijk welk type (elektromagnetisch, elektrostatisch of piëzo-elektrisch) het meest geschikt is voor sonde data-opslag. We hebben de elektrodynamische actuatoren in het scanner prototype van IBM vervangen met comb drives, om een directe vergelijking mogelijk te maken (hfst. 3). De comb drives zijn relatief erg groot, te wijten aan een relatief lage elektrostatische kracht. Om de gegenereerde kracht te verhogen, hebben we de vorm van de comb drive vingers geoptimaliseerd voor schokbestendige actuatie in een sonde data-opslag systeem (hfst. 4).

De geschiktheid van elektromagnetische en elektrostatische actuatie wordt onder andere bepaald door hun energieverbruik (hfst. 5). Er zijn drie (gedeeltelijk) hypothetische scanner ontwerpen gemaakt, met elektrodynamische, elektromagnetische en elektrostatische comb drive actuatie. De prestaties zijn ongeveer gelijk, maar elektrostatische comb drives verbruiken een orde grootte minder energie. Er zijn formules opgesteld voor verder onderzoek naar de prestaties en het energieverbruik voor verschillende bestandssystemen.

Hoofdstuk 6 beschrijft ons micro-instrument het 'micronium': een instrument dat geluid maakt m.b.v. MEMS structuren. Omdat het fabricage proces onvoldoende nauwkeurig is, is het instrument vals en moet het gestemd worden. Naast de leuke kennismaking voor studenten met MEMS-technologie, heeft het micronium MEMS-technologie voor een breder publiek toegankelijk gemaakt. 


\section{Publications}

The research presented in this thesis has lead to the following publications.

\section{Journal articles}

Engelen J B C, Rothuizen H E, Drechsler U, Stutz R, Despont M, Abelmann L, Lantz M A, 2009 "A mass-balanced through-wafer electrostatic $x / y$-scanner for probe data storage"

Microelectron. Eng. 86, pp. 1230-1233, doi:10.1016/j.mee.2008.11.032

Engelen J B C, Abelmann L, Elwenspoek M C, 2010

"Optimized comb-drive finger shape for shock-resistant actuation"

J. Micromech. Microeng. 2o, p. 105003, doi:10.1088/0960-1317/20/10/105003

Three articles are soon to be published: an article on the energy consumption of different microactuator types for probe data storage (Chapter 5), a technical article on the micronium (Chapter 6), and a more popular article about the micronium in the Dutch Nederlands Tijdschrift voor Natuurkunde.

\section{Book chapters}

Gemelli M, Abelmann L, Engelen J B C, Khatib M G, Koelmans W W, Zaboronski O, [in press] 2011 Memory Mass Storage, chapter Mass Storage using Millipede Technology,

Springer-Verlag

ISBN 978-3-642-14751-7

\section{Conference contributions}

Brouwer D M, Otten A, Engelen J B C, Krijnen B, Soemers H M J R, 31 May-4 Jun. 2010 "Long-range elastic guidance mechanisms for electrostatic comb-drive actuators" In: Proc. 1oth Int. Conf. of the European Society for Precision Engineering \& Nanotechnology (euspen 2010), pp. 47-50, Delft, the Netherlands

Delalande M Y, Engelen J B C, le Fèbre A J, Abelmann L, Lodder J C, Dec. 2008 "Switching field distribution of arrays of Co-Pt nanodots determined by anomalous Hall effect measurements"

In: Proceedings of the Asian Magnetics Conference 2008, Busan, Korea

Delalande M Y, Engelen J B C, le Fèbre A J, Abelmann L, Lodder J C, Apr. 2009

"Thermally induced switching field distribution of individual single nanomagnets in a large assembly"

In: Proceedings of the European Workshop on Self-Organized Nanomagnets, p. 3o, Aussois, France 
Engelen J B C, Bolhuis T, Abelmann L, Lodder J C, 18-20 Jun. 2007

"Anomalous Hall effect measurements on a nanosized CoPt dot array"

In: Innovative Mass Storage Technologies (IMST 2007), Enschede, the Netherlands

Engelen J B C, Lantz M A, Rothuizen H E, Abelmann L, Elwenspoek M C, 21-25 Jun. 2009 "Improved performance of large stroke comb-drive actuators by using a stepped finger shape" In: Int. Solid-State Sensors, Actuators and Microsystems Conf. (TRANSDUCERS 2009), pp. 17621765, Denver, CO, USA, doi:10.1109/SENSOR.2009.5285744

Engelen J B C, Abelmann L, Elwenspoek M C, 24-28 Jan. 2010 "Optimized comb drive finger shape for shock-resistant actuation" In: 23 rd Int. Conf. on Micro Electro Mechanical Systems (MEMS 2010), pp. 1147-1150, Hong Kong, China, doi:10.1109/MEMSYS.2010.5442414

Engelen J B C, de Boer H, Beekman J G, Been A J, Folkertsma G A, Fortgens L, de Graaf D, Vocke S, Woldering L A, Abelmann L, 26-29 Sept. 2010

"A musical instrument in MEMS"

In: Proc. Micromechanics and Microsystems Europe, Enschede, the Netherlands

Hogervorst R P, Krijnen B, Brouwer D M, Engelen J B C, Staufer U, 31 May-4 Jun. 2010 "A single-mask thermal displacement sensor in MEMS"

In: Proc. 1oth Int. Conf. of the European Society for Precision Engineering \& Nanotechnology (euspen 2010), pp. 462-465, Delft, the Netherlands

Krijnen B, Hogervorst R P, Engelen J B C, van Dijk J W, Brouwer D M, Abelmann L, 26-29 Sept. 2010 "Single-mask thermal displacement sensor in MEMS"

In: Proc. Micromechanics and Microsystems Europe, Enschede, the Netherlands

Yntema D R, Haneveld J, Engelen J B C, Brookhuis R A, Sanders R G P, Wiegerink R J, Elwenspoek M, 24-28 Jan. 2010

"Listening to MEMS: An acoustic vibrometer"

In: Proc. IEEE MEMS 201o, pp. 663-666, Hong Kong, China, doi:10.1109/MEMSYS.2010.5442319 


\section{Dankwoord}

Bij het maken van een lijst met namen kom ik er achter dat ik nooit iedereen recht kan doen in slechts een paar paginas. De lijst alleen is al bijna een hele pagina. Ik zal me beperken tot een hak-op-de-tak verzameling van mijn beste herinneringen aan de mensen die wat directer betrokken zijn geweest bij mijn promotie. Lloret de Bar en huisgenoten Karel en Oane, Ludica, Slagvaardig, Duck4Cover, PaperJam, Sad Scientists, bedankt voor de momenten dat het lukte om wetenschap even uit mijn hoofd te zetten.

Pa en ma, bedankt voor mijn studie, weinig vragen en veel begrip.

Ik kan mij geen fijnere begeleider voorstellen dan Leon Abelmann. Als 'assistent in opleiding' was ik heel blij met de letterlijke interpretatie hiervan. Altijd kon ik binnenstormen met een vraag of iets leuks. Het bezoek aan IBM, de twee studiereizen, het opzetten van een nieuw vak, het micronium, enzovoorts. Dat ik mag slapen, dat ik te laat mag komen en mag vergeten, dat ik af mag dwalen, dat ik mag spelen. En dat het dan uiteindelijk toch iets oplevert. Heel erg bedankt Leon, zonder jou was het niet $z o$ geweest. Ik ben heel blij dat er een goede kans is dat wij elkaar in de toekomst nog veel zullen treffen.

Vanwege de opsplitsing en fusie van vakgroepen, heb ik voor mijn gevoel bijna twee promotoren. Cock, achteraf gezien lijkt het bizar dat je niet in mijn commissie zit. Ik probeer me te houden aan je advies, "pas op dat je geen manager wordt." Miko, mijn beste herinnering is van ver voor mijn promotie: het bevlogen college over Feynman zo ongeveer 10 jaar geleden. De vonk is overgeslagen!

Bedankt kamergenoten, Regina, Jeroen en natuurlijk Wabe, voor het aanhoren van mijn verhalen en dat ik jullie van je werk mocht houden. Wabe, één beste herinnering is lastig..., misschien het openen van de mistwolken op de Franse berg met Harald? Of toch de barre tocht naar de soep op Rigi? Helaas moeten we afscheid nemen, maar wellicht slechts voor korte tijd...

De SMI koffietafel met o.a. Alexander, Mink, Ivan, en Michel heb ik gemist. Gelukkig kwam er een bank voor in de plaats in de leukste UT vakgroep met 'sexy collega's' Marcus, Doekle en Jeroen, en andere collegae Hadi, Shahina, Harmen, Laurens, Hammad, Florian, Ahmad, ... (lees verder)

Robert, ik ben blij dat wij nooit op hetzelfde kantoor hebben gezeten.

Mohammed, uiteindelijk is het toch gelukt samen een artikel te schrijven.

Michael, je suis fier de notre article, tu devrais être le premier auteur aussi.

Dannis en Bram, bedankt dat ik me heb mogen bemoeien met jullie onderzoek. 
Conferentie reizen waren fantastisch dankzij mijn medereizigers. Het huisje in een klein dorpje bij Lyon, met Nima, Ram, Wabe, Dennis, Gijs, en Arjan. Nog steeds vertel ik verhalen over de tartaar en het verzoek om een "well done" entrecôte bij Brasserie Georges (Ram?), en ik zal de vertaling van duct tape in het Frans ook niet snel vergeten. Athene, met Marjolein, Dennis, Yiping, Pieter en Ram (remember our talk about ants?); en natuurlijk het reisje naderhand met Marcel, Cyrill en Robbie. Denver, de honkbalwestrijd, ja toch Gijs! Hong Kong..., Remco, Ciska, Doekle, Jeroen, Maartje, en Edin, niet bedankt voor de avond stappen, wel voor de 'piano dames' en de helikopter.

Comb drives maken kan $i k$ niet. Mijn eerste stappen in de cleanroom waren met Johnny samen, later met Léon en Joël. En uiteindelijk lukt het Kees wèl met fatsoenlijke yield. Léon, jouw scherpe kritiek hield me op het rechte pad (ik heb zo snel geen referentie voor dit feit). Peter, bedankt voor de maskers, ook als ik weer te grote polygonen had of een correctie op de correctie moest doen. Meint, Erwin, bedankt voor de adviezen maar vooral voor de humor en de scherpe gesprekken.

Thijs, Martin, Pino, Henk, Marcel, altijd stonden jullie voor mij klaar. En dan hebben jullie altijd toch weer een handigere oplossing. De PinoTech Vacusuck is geniaal, en foto's maken met tilt-shift (niet uit de losse hand!) ga ik missen.

Karen, Sati, Susan, Thelma, wat moeten wij zonder jullie? Ik: "Wanneer heb ik ook al weer de vaste afspraken met Leon staan?" Karen: “Gisteren ;-)” Bijzonder hoe je het uithoudt met ons (ik) chaoten!

Remco, Niels, Vitali, Joost, Theo, bedankt voor jullie bijgedragen aan mijn werk. Henri, Faraday's paradox blijft tot nog toe een hersenkraker, bedankt!

Een aantal studenten heb ik mogen begeleiden en meehelpen met hun IOO, stage, en/of afstudeer projecten. Thomas, Jarno, Jaap, Esken, Harald, Mathijs, Richard, Alexander, Jan-Willem, bedankt voor nieuwe inzichten. Twee, wat? drie! B2 projecten heb ik mee mogen begeleiden. Marco, Olti, Ewout, Stefan, bedankt voor de leuke acht weken. Rick, Wouter, Daniël, Diederik, de demo is af hoor en gaaf! Laurens, Sander, Jethro ('jètro', niet 'jétro'), Derk, Auke, Geert, Hylco, Jasper, zonder jullie geen micronium. Arvid, Daria, Hans, door jullie is het een groot succes geworden on stage en op internet.

My stay at IBM ZRL kicked my PhD into gear and was good fun, thanks Michel, Mark, Hugo, Ute, Richard, Rachel, Harish, Abu, Evangelos, Martin, Silvia, and also the people outside the data storage group, Cyrill, Robbie, Emmanuel, Elly, and Luc. Fortunately, I will be seeing (most of) you soon again!

Roderick: "Wil je mee op studiereis?" Ik: "Nee joh, je wilt helemaal geen AiO mee hebben.” R: “Jawel!” Ohiariha (Joost, Tom, Roderick, Martin, Tomas, ...) en Ngò’i Sáng (Lars, Ewout, Marco, Wesley, Harm, Tom, ...), bedankt voor fantastische herinneringen en het herleven van mijn eigen hoogtij dagen, al heb ik niet meer bijgedragen dan het maken van de winnende foto en wat gitaar spelen...

Bedankt allen! Pas bij het schrijven van deze tekst dringt het tot me door dat ik binnenkort vertrek, op lange afstand ga wonen, en jullie gezelschap moet missen... Heu! 


\section{Biography}

Johan Engelen was born in Nijmegen, the Netherlands on 8 June, 1983. He attended primary school in Gendt, the Netherlands, and went to het Stedelijk Gymnasium in Nijmegen, the Netherlands, to obtain the VWO diploma. He did his first research project together with Leonard Raymakers, investigating tilted roller-coaster loops, for which they received special honours at the presentation of the Van Melsen awards in 2000. That year, Johan started the Electrical Engineering course at the University of Twente. He obtained his first-year diploma cum laude. Together with 6 other students, he organised the Shouraizou studytour project (2003-2005), carrying the responsibility of organising travel, accommodations and cultural excursions in Japan, and arranging visits to Japanese companies, institutions and universities. From November 2005 to March 2006, Johan stayed in Lincoln, Nebraska, USA, investigating electron transport in large magnetic fields in David Sellmyer's group in the Department of Physics and Astronomy at the University of Nebraska-Lincoln. Back at the University of Twente, in the Systems and Materials for Information storage (SMI) group led by Cock Lodder, he investigated the magnetisation reversal of 'nanodots' in arrays using the Anomalous Hall Effect, with which he graduated cum laude in 2006. He then started as a Ph.D. student on the research described in this thesis.

\section{Biografie}

Johan Engelen werd geboren op 8 juni 1983, te Nijmegen. Hij doorliep de lagere school in Gendt, waarna hij in 1994 naar het Stedelijk Gymnasium in Nijmegen ging. Zijn eindexamen onderzoek naar gekantelde achtbaan loopings samen met Leonard Raymakers werd beloond met een eervolle vermelding bij de uitreiking van de Van Melsen prijs in 200o. In hetzelfde jaar begon Johan zijn studie tot electrotechnisch ingenieur aan de Universiteit Twente. Hij behaalde de propedeuse in het eerste jaar cum laude. In een groep van 7 studenten organiseerde hij het Shouraizou studiereisproject naar Japan (2003-2005), waarin hij verantwoordelijk was voor vervoer, accomodatie en culturele uistapjes in Japan, en voor het contact met de bezochte Japanse bedrijven, instituten en universiteiten. In de groep van David Sellmyer in the Department of Physics and Astronomy aan the University of Nebraska-Lincoln deed Johan een stageonderzoek naar electrische stromen in magnetisch velden, van november 2005 tot maart 2006, in Lincoln, Nebraska, Verenigde Staten. In Cock Lodder's Systems and Materials for Information storage (SMI) groep aan de Universiteit Twente deed hij onderzoek naar het omschakelen van de magnetisatie van 'nanodots' in arrays met behulp van Anomale Hall Effect metingen, waarmee hij cum laude afstudeerde in 2006. Daarna begon hij als beoogd promovendus aan het onderzoek beschreven in dit proefschrift.

E-mail: j.b.c.engelen@alumnus.utwente.nl 


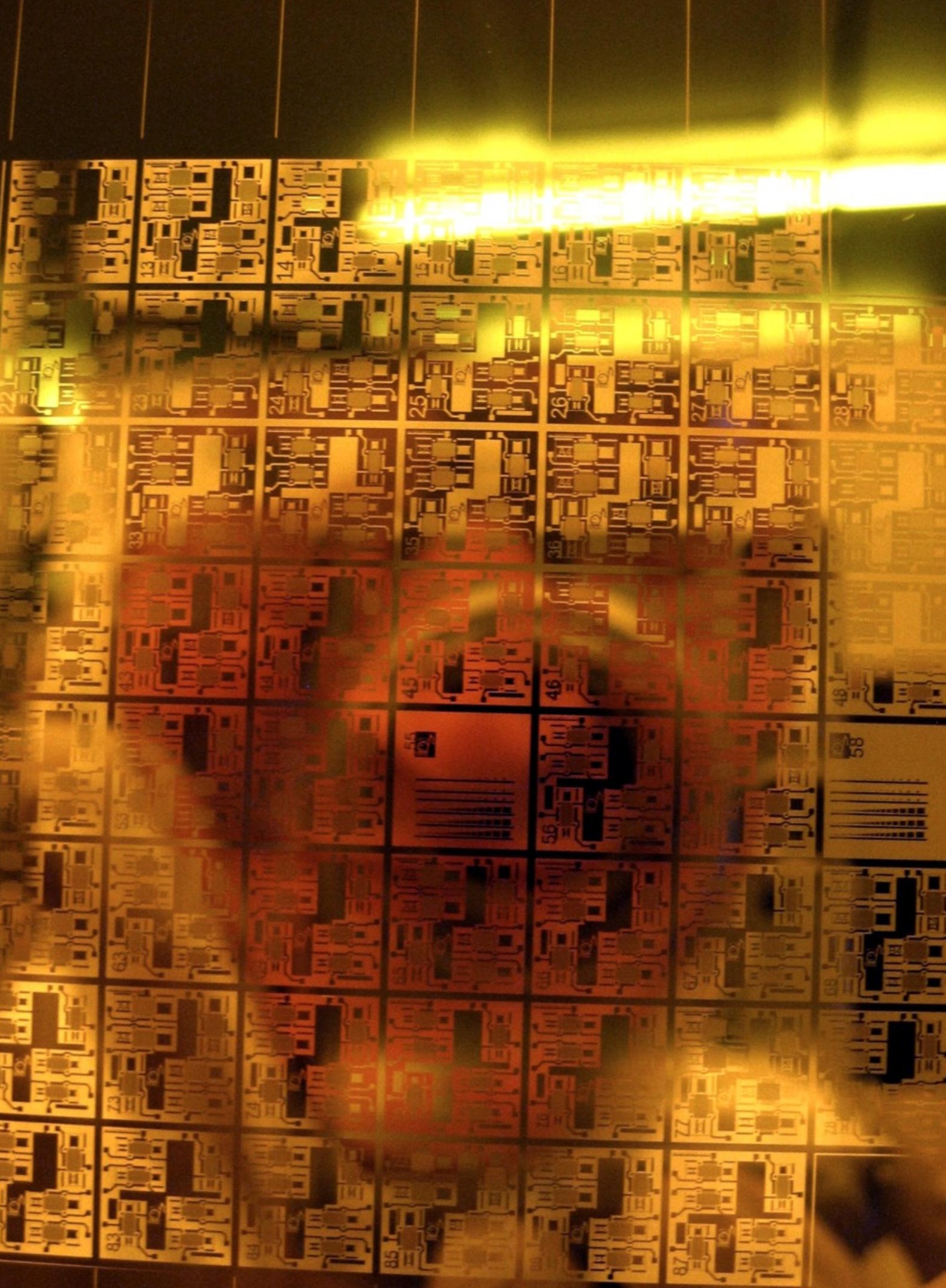

